\title{
Hierarchical Mechanics of Connective Tissues: Integrating Insights from Nano to Macroscopic Studies
}

\author{
Kheng Lim Goh ${ }^{1, *}$, Anne Listrat ${ }^{2}$, and Daniel Béchet ${ }^{3}$ \\ ${ }^{1}$ School of Mechanical and Systems Engineering, Newcastle University, Newcastle, UK \\ 2 INRA Centre de Clermont Ferrand Theix UMRH équipe AMUVI 63122 Saint Genès Champanelle, France \\ ${ }^{3}$ INRA, UMR 1019, UNH, CRNH Auvergne, F-63122, Saint Genels Champanelle, France
}

\begin{abstract}
As the key component of the musculoskeletal system, the extracellular matrix of soft connective tissues such as ligaments and tendons is a biological example of fibre-reinforced composite but with a complex hierarchical architecture. To establish a comprehensive structure-function relationship at the respective levels (i.e., from molecule to tissue) of the hierarchical architecture is challenging and requires a multidisciplinary approach, involving the integration of findings from the fields of molecular biology, biochemistry, structural biology, materials science and biophysics. Accordingly, in recent years, some of these fields, namely structural biology, materials science and biophysics, have made significant progress in the microscale and nanoscale studies of extracellular matrix using new tools, such as microelectromechanical systems, optical tweezers and atomic force microscopy, complemented by new techniques in simultaneous imaging and mechanical testing and computer modelling. The intent of this paper is to review the key findings on the mechanical response of extracellular matrix at the respective levels of the hierarchical architecture. The main focus is on the structure and function-the findings are compared across the different levels to provide insights that support the goal of establishing a comprehensive structure-function relationship of extracellular matrix. For this purpose, the review is divided into two parts. The first part explores the features of key structural units of extracellular matrix, namely tropocollagen molecule (the lowest level), microfibril, collagen fibril, collagen fibre and fascicle. The second part examines the mechanics of the structural units at the respective levels. Finally a framework for extracellular matrix mechanics is proposed to support the goal to establish a comprehensive structure-function relationship. The framework describes the integration of the mechanisms of reinforcement by the structural units at the respective levels of the hierarchical architecture in a consistent manner, both to allow comparison of these mechanisms and to make prediction of the interconnection of these mechanisms that can also assist in the identification of effective mechanical pathways. From a design perspective, this is a step in the direction towards the development of effective strategies for engineering materials to replace or repair damaged tissues, and for exogenous cross-linking therapy to enhance the mechanical properties of injured tissues.
\end{abstract}

KEYWORDS: Tropo-Collagen Molecule, Microfibril, Collagen Fibril, Proteoglycan, Hierarchical Architecture, Human Physiome Project.

\section{CONTENTS}

Introduction .

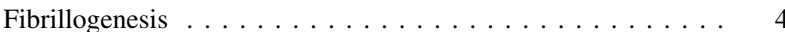

Fibril-Associated Proteoglycans ........................ 5

Low Dimensional Structural Units . . . . . . . . . . . . . 8

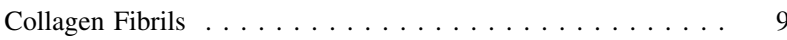

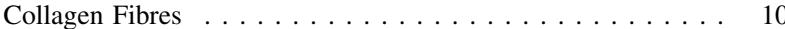

\footnotetext{
${ }^{*}$ Author to whom correspondence should be addressed.

Email: kheng-lim.goh@newcastle.ac.uk

Received: 3 February 2014

Accepted: 1 March 2014
}

Summary $\ldots \ldots \ldots \ldots \ldots \ldots \ldots \ldots \ldots \ldots \ldots \ldots$

Extracellular Matrix (ECM) Function . . . . . . . . . . . . . . . . . 11

Mechanical Response Graphs . . . . . . . . . . . . . . . . 11

Tropo-Collagen Molecular Mechanics . . . . . . . . . . . . 13

Collagen Fibril Mechanics . . . . . . . . . . . . . . . . . . . . . 18

Collagen Fibre Sliding Mechanics . . . . . . . . . . . . . . 28

Elasticity and Fracture Toughness of Structural Units . . . . . . 29

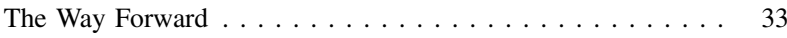

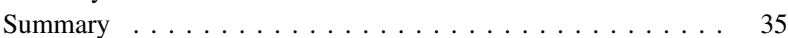

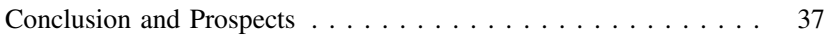

Appendix . . . . . . . . . . . . . . . . . . . . . 38

Acknowledgments . . . . . . . . . . . . . . . . . . . . . . . . . . . . . . . . . 39

References . . . . . . . . . . . . . . . . . . . . 39

$1550-7033 / 2014 / 10 / 001 / 044$

doi:10.1166/jbn.2014.1960 


\section{INTRODUCTION}

The basic building blocks of extracellular matrix (ECM) of soft connective tissues (SCTs), such as tendons and ligaments, are collagen, proteoglycans (PGs) and elastin. ${ }^{1-3}$ These are structural proteins which can organize into long structural units at different length scales in ECM with collagen predominating at all levels. ${ }^{4,5}$ The entire organisation has been described as having a hierarchical architecture. This description is not based on any theoretical analysis but it indicates that there are several levels spanning different length scales. Thus, for the unidirectional SCTs such as tendons and ligaments, the lowest level has been identified with the structural unit, tropo-collagen (TC) molecule. Thereafter, in order of increasing level up to the whole tissue, the structural units identified with the other levels are microfibril, collagen fibril, collagen fibre and fascicle. A simple schematic of the hierarchical architecture of ECM that emphasizes the structural unit at the respective levels is shown in Figure 1(A). Although the hierarchical architecture of some SCTs-particularly the unidirectional ones such as tendons and ligaments-are better known than others, no consensus has been reached with regard to the number of levels that made up ECM. For the caveats, these levels were identified, somewhat artificially, within the limitations of the instruments used in the studies. ${ }^{6}$

In spite of the fact that the hierarchical architecture of ECM has been a subject of intense discussion, 2,4,5,7-11 we still do not have a comprehensive understanding of the structure-function relationship that links all levels.

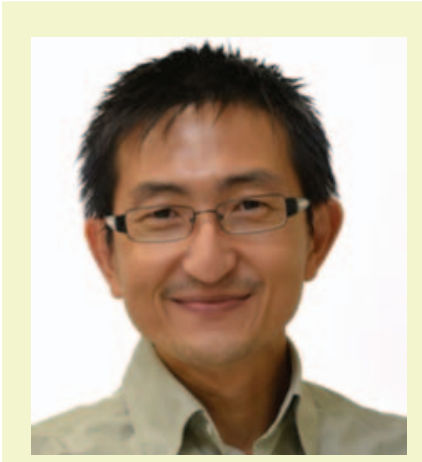

Kheng Lim Goh, is a senior lecturer at the School of Mechanical and Systems Engineering, Newcastle University (UK). He is seconded, as Director of Operations, to NUInternational Singapore Pte Ltd. (NUIS) to run the mechanical design and manufacturing engineering degree programme. He holds a B.Sc. degree (Physics), an M.Sc. degree (Medical Physics) and a Ph.D. degree (Bioengineering). He is a member of Institute of Physics (IOP), Institute of Physics and Engineering in Medicine (IPEM) and Institute of Mechanical Engineers (IMechE). He is a Chartered Physicist (CPhys), Scientist (CSci) and Engineer (CEng). His research aims to understand the physical properties of natural and synthetic materials and to use this understanding to design composite biomaterials for biomedical engineering applications. At Newcastle University, he is involved in developing new micromechanical systems for probing extracellular matrix of soft connective tissues.

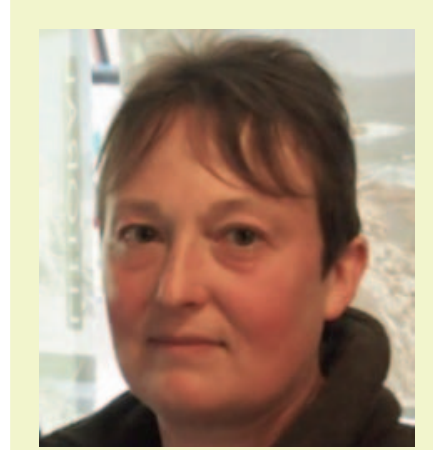

Anne Listrat is a french reseacher of the National Institute of Agronomical Research. She holds a Ph.D. in Biology and Food Science on the subject: IGFII mRNA and type I and II receptors localization in the bovine muscle tissue during its fetal differentiation. She is certified to supervise Research by Clermont II University (France). At the National Institute of Agronomical Research, in Unit of Research on the Herbivores, she explores the extracellular matrix (ECM) characteristics involved in meat quality of bovine meat. She has also studied ontogenesis and impact of breeding factors on ECM characteristics and the ECM role in muscle aging. She is author and coauthor of 34 original papers and she has supervised 15 graduate students (Master Degree and Ph.D. students).

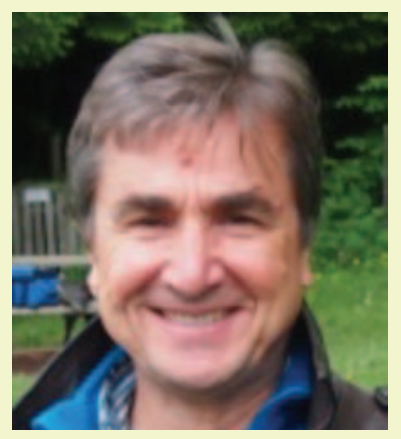

Daniel Béchet, a native of Riom, France, is Research Director and a principal investigator in the Department of Human Nutrition of INRA (National Institute of Agronomic Research), CRNH (Human Center of Human Nutrition) and Auvergne University in Clermont-Ferrand (France). He holds a B.SC. degree in Physiology and Genetics, an M.Sc. degree in Biochemistry (Clermont-Ferrand, France), an engineering school degree in Physical Chemistry (Lyon, France), and a Ph.D. degree in Endocrinology from Bristol University (UK). He is a research member of UMR-1019, INRA, and has explored the mechanisms of expression of lysosomal cathepsins in skeletal muscle. He determined the amino acid sequence of cathepsin L, the gene structure of cathepsin B and investigated the signaling pathways regulating autophagy in muscle cells. Currently, he investigates the mechanisms of muscle aging (sarcopenia) in human cohorts using transcriptomics, proteomics, and mass spectrometry molecular imaging. 

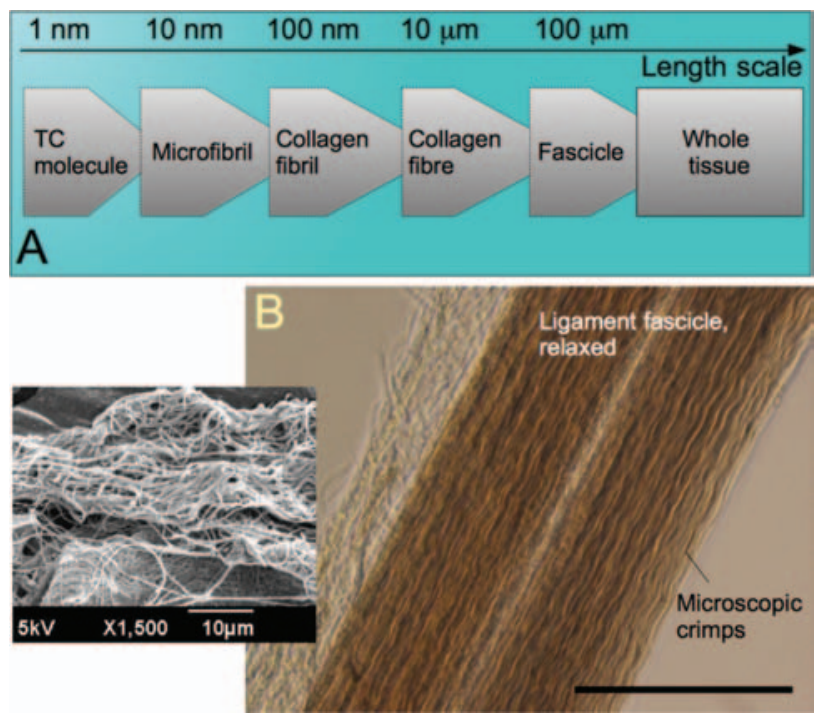

Figure 1. Structure of soft connective tissues. (A) A model depicting the length scale of the structural units of extracellular matrix at the respective levels of soft connective tissues, e.g., tendon and ligament. The more commonly used models would depict sketches of the respective structural units in a zoom-in manner. ${ }^{191}$ Of note, the diameter of a TC molecule is $\approx 2 \times 0.28=0.56 \mathrm{~nm} .{ }^{14,15}$ The diameter of the microfibril is $\approx 3.5 \mathrm{~nm}^{72}$ (B) Optical micrographs of an isolated filamentary ligament fascicle from bovine anterior cruciate ligament (Scale bar: $100 \mu \mathrm{m})$. Inset shows a scanning electron micrograph of the collagen fibrils on the surface of a ruptured fascicle (unpublished data from an earlier study). ${ }^{27}$

In recent years, several fields, namely structural biology, biophysics and materials science, have witnessed significant progress in microscale and nanoscale studies of ECM using state-of-the-art tools for low dimensional tensile testing (i.e., uniaxial extension), namely microelectromechanical systems (MEMS), ${ }^{12,13}$ optical tweezers ${ }^{14,15}$ and atomic force microscopy (AFM) ${ }^{16}$ For structural analysis, the new tools are confocal microscopy, ${ }^{8,9,17} \mathrm{X}$-ray crystallography from synchrotron light sources ${ }^{10}$ and three-dimensional electron computer tomography. ${ }^{18}$ Complementing these new tools are new techniques in strain analysis, e.g., local (microscopic) strain measurement by simultaneous imaging and mechanical testing $8,9,17$ and mapping of molecular structural changes under mechanical loading by Raman spectroscopy, ${ }^{19}$ as well as new techniques in computer modeling by molecular dynamics (MD) simulation. ${ }^{5}$ Altogether, this presents an opportunity to revisit the findings derived from these studies, as well as other related studies, to gain further insights into the interconnection of the different structural units for reinforcing ECM.

The scope of this review addresses the key findings on the structure and the (mechanical) function of ECM. For simplicity, we draw on the findings from two unidirectional SCTs, namely tendons and ligaments, which are characterized by highly paralleled collagen fibrous units (Fig. 1(B)). Accordingly, it can be argued that these tissues are analogous to engineering fibre composites where fibres are laid down in parallel for directional reinforcement. Although tendons and ligaments are structurally very similar-the highly paralleled collagen fibres feature is found in both tissues - differences in the anatomical locations of tendons (i.e., bridging muscle to bone) and ligaments (i.e., bridging bone to bone) mean that they serve different functions in the musculoskeletal system. ${ }^{20}$ Thus the tendon transmits load generated by the muscle (during contraction) to the bone to enable joint movement. ${ }^{20}$ Ligaments provide mechanical stability (being shorter than tendons) by constraining and guiding joint motion through tensile and torsional loading action. ${ }^{20}$ In principle, the arguments developed in this paper could apply, somewhat, to non-unidirectional tissues such as cartilage which contain randomly oriented fibrous structures - when an external load acts on the tissue, these fibrous structures will be recruited into tension by realigning their axes in parallel to the direction of the load. For the same purpose, the emphasis will be on the findings from mechanical testing (uniaxial extension) of whole tissues, or single fascicles, single collagen fibrils and single collagen molecules derived from uniaxial SCTs to help establish insights into the mechanisms of reinforcement of the tissue.

In accordance with the scope of this review we have divided the report into two parts, namely tissue structure and function. For the first part, the discussion focuses on the findings that address key structural units at the different levels within the hierarchical architecture, namely TC molecule (the lowest level), microfibril, collagen fibril, collagen fibre, fascicle and finally whole tissue. The second part examines how the structural units at the respective levels respond to external loads, with reference to tensile testing from initial loading until the tissue ruptures. Finally, the findings discussed in this review are organized within a framework for ECM mechanics. The framework describes the various mechanisms of reinforcement by the structural units at the respectively levels of the hierarchical architecture, at different stages of the loading process, in a consistent manner both to allow comparison of these mechanisms and to make prediction of the interconnection of these mechanisms that can also assist in the identification of new mechanical pathways. The goal is to establish a comprehensive understanding of the structure-function relationship linking all levels. From a design perspective, this is a step in the direction towards the development of effective strategies for optimizing engineering materials for use in tissue regeneration. ${ }^{6,21}$ From a clinical science perspective, the framework is a step in the direction for illuminating the causes of alteration in ECM organisation arising from degeneration ${ }^{22-24}$ and injury, ${ }^{22,25,26}$ that could compromise the mechanical integrity of SCTs, and for developing effective strategies to combat the changes in ECM organization, as well as to enhance the mechanical properties of SCTs via therapeutic procedures. $^{25-27}$ 


\section{EXTRACELLULAR MATRIX (ECM) STRUCTURE}

\section{Fibrillogenesis}

Collagen protein comprises three polypeptides bound together to form a supercoiled triple helix molecule which we have termed as a TC molecule. ${ }^{28-30}$ Each polypeptide is described by a Gly-X-Y motif. Here, Gly refers to glycyl residue; $\mathrm{X}$ and $\mathrm{Y}$ can be any residue but are usually represented by the proline (Pro) residue and 4-hydroxyproline (Hyp) residue, respectively. ${ }^{28-30}$ Further details concerning the structural features of TC molecules will be addressed in a later discussion (see Section Tropocollagen molecules). Since collagen dominates ECM of SCTs such as ligaments and tendons, we shall discuss the synthesis process of collagen as the starting point for addressing our understanding of the hierarchical architecture.

Studies of the molecular basis of collagen formation into fibrillar structures, otherwise known as fibrillogenesis, ${ }^{31-34}$ seek to understand how the mechanism of nucleation, growth (axial and lateral) and remodeling (e.g., of fracture ends) produce collagen fibrils. Consequently, these studies have provided insights into the trafficking of procollagenthe precursor of collagen - through the cellular secretory pathway, the conversion of procollagen to collagen by the procollagen metalloproteinases, and the directional deposition of fibrils (i.e., laying down and orientating the fibrils) involving the plasma membrane and late secretory pathway. The trafficking mechanisms of ECM components from cells into ECM are thought to be similar in eukaryotes. ${ }^{30,35}$ The key stages of the synthesis process from procollagen to collagen fibrils are illustrated in Figure $2 .^{36,37}$ At the beginning of the fibril synthesis process, chains of procollagen molecules are folded into rodlike triple-helical procollagen trimers in the endoplasmic reticulum (the synthesis sites). ${ }^{38} \mathrm{Next}$, the reduction process of procollagen trimers-which involves the removal of the globular $\mathrm{N}$ - and $\mathrm{C}$-propeptides from procollagen by the $\mathrm{N}$ - and C-proteinases ${ }^{36,37}$-yields TC molecules. ${ }^{39}$ Thereafter, the secretion process occurs, involving the transportation of TC molecules from the cell through the Golgi to the plasma membrane (PM) via carriers known as the Golgi-to-PM compartments (GPCs). The mission of GPCs involves the migration into the PM and fusion with the PM to form a fibril depositor (or otherwise known as fibripositor). Fibripositors are long structures located at the side of the cell, aligned along the long axis of SCTs such as tendon and ligament, projecting into ECM where the collagen fibrils are found..$^{29,33,36,37,40}$ Alternatively the GPCs may also fuse with the base of existing fibripositors. Accretion of fibrils then occurs in the fibripositor by the addition of individual collagen molecules to the fibril; this may be initiated at the ends and centre of the fibrils. Thereafter, nucleation propagates along the surface, generating a smoothly tapered profile; the overall length and cross-sectional size increase
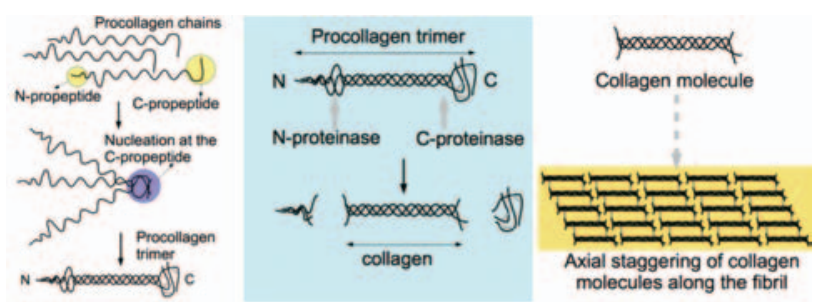

A

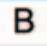

C

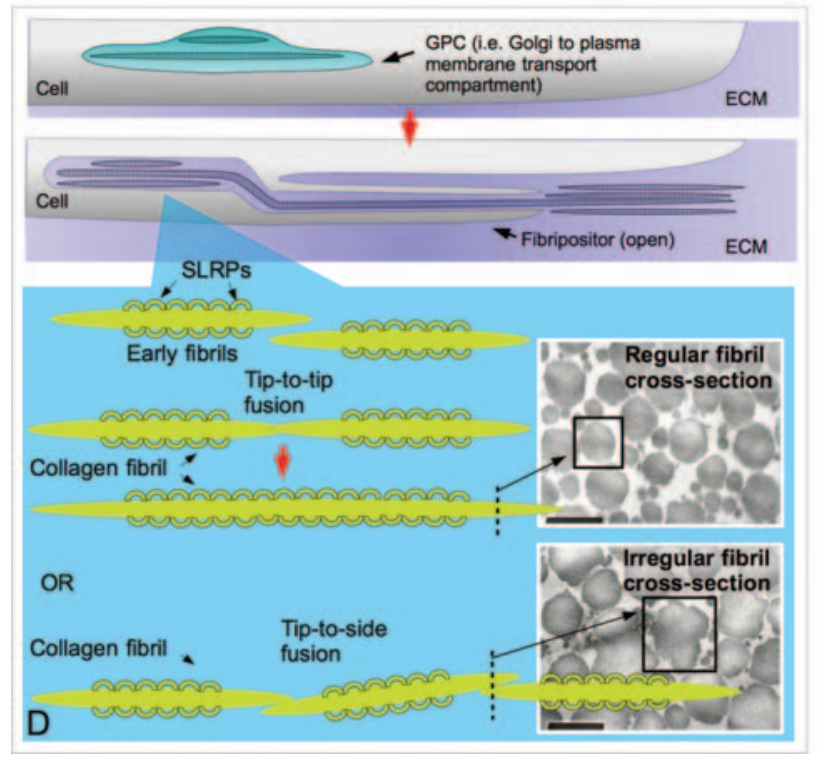

Figure 2. Synthesis of collagen fibrils. (A) Procollagen chains are produced in the endoplasmic reticulum. Next, the C-propeptides interact and fold (nucleation at the C-propeptide) followed by the $\mathrm{N}$-propeptides. The final form is a rod-like triple-helical domain flanked by globular $\mathrm{N}$ and C-propeptides. C: carboxyl; N: amino (B) Procollagen trimer is cleaved at the $\mathrm{N}$ and $\mathrm{C}$ propeptides ends by $\mathrm{N}$ and $\mathrm{C}$ proteinase, respectively. This yields the ubiquitous right-handed coiled triple helical tropo-collagen (TC) molecule. It is thought that the removal of the $\mathrm{N}$ - and $\mathrm{C}$-propeptides occurs after procollagen has trafficked through the Golgi apparatus. (C) TC molecules assemble, held together by covalent cross-links, into fibrous structures known as collagen fibrils. (D) Schematics of collagen fibrils in a Golgi-to-PM transport compartment (GPC), followed by collagen fibrils in an 'open' fibripositor, which is aligned in the direction of the axis of, e.g., tendon or ligament. It is thought that collagen fibrils may grow in the fibripositor from the tips by tip-to-tip or tip-to-side fusion. . $^{43,47}$ Figures in panel (A) to (D) are adapted with permission from [36 and 37], E. G. Canty, et al., Coalignment of plasma membrane channels and protrusions (fibripositors) specifies the parallelism of tendon. J. Cell Biol. 165, 553 (2004). @ 2004; E. G. Canty and K. E. Kadler, Procollagen trafficking, processing and fibrillogenesis. Journal of Cell Science 118, 1341 (2005). ○) 2005. Insets in panel (D) are transmission electron micrographs of tail tendons (mice) showing the cross-section of the collagen fibrils from a young and an old individual. Scale bar: $300 \mathrm{~nm}$; unpublished data from an earlier study. ${ }^{189}$

until the axial length reaches $\approx 13 \mu \mathrm{m} .{ }^{41-44}$ The fibril assembly process is thought to be entropic-driven. ${ }^{33,36,37}$ TC molecules are bound to the fibrils by cross-links, located at the $\mathrm{C}$-terminal and $\mathrm{N}$-terminal of the molecule. ${ }^{45}$ 
The implications of these cross-links on the mechanical properties of TC molecules will be left for a later discussion (see Section Role of cross-links). It is speculated that further fibrillar growth thereafter is by tip-to-tip fusion and/or tip-to-side fusion. ${ }^{33,46}$ Although these different modes of growth could occur in the fibripositor as well as outside the cell, ${ }^{33,46}$ the mechanism regulating fibril fusion is not well understood. Thus, short fibrils may also be found within a fibripositor. ${ }^{29,33,36,37,40}$ On the other hand, longer fibrils are positioned with one end in the cell (while the other end sticks out of the cell), and the bulk of the fibril is enclosed within the fibripositor. ${ }^{29,33,36,37,40}$ This begs the question of the stage in which the fibril begins to contribute to reinforcing ECM. Unfortunately, the mechanical properties of these early collagen fibrils are not well understood and could be a subject for further investigation.

To continue the discussion on the fibrillar growth by fusion in ECM, first we reiterate that axial growth at later stages of the fibril could occur by tip-to-tip fusion and lateral growth could occur by tip-to-side fusion (Fig. 2(D)). ${ }^{46}$ Evidence for fibrillar growth by tip-to-tip fusion could be found in images of fibrils seen from the transverse sections of tendons in micrographs taken by transmission electron microscopy (TEM) ${ }^{31}$ and from entire (isolated) fibril imaged by dark-field scanning TEM (STEM), complemented by measurements of the axial mass distribution. ${ }^{46}$ It is speculated that tip-to-side fusion could result in a fibril with irregular cross-section. ${ }^{47}$ To the best of our knowledge, this could not be confirmed because no investigations have been carried out to observe the growth of fibrils in situ. Nevertheless, the presence of short fibrils (length $\sim 7-15 \mu \mathrm{m}$ ) in electron micrographs implies that these could be the precursors in collagen fibril formation, with longer fibrils forming by the fusion of the shorter ones. ${ }^{31}$ Second, we note that early collagen fibrils are either (1) unipolar (with carboxyl (C) and amino (N) ends) or (2) bipolar fibrils (with two N-ends). ${ }^{46}$ In particular, for the bipolar fibrils, there is a transition region (i.e., equivalent to a mirror-symmetric plane) spanning eight $D$ periods along the shaft in which the TC molecule flips $180^{\circ}$ so that only the $\mathrm{N}$ terminus of all $\mathrm{TC}$ molecules point towards the ends. Our current understanding of how fibrils fused has suggested that the polarity of the fibrillar end and PGs are key contributory factors to fibrillar fusion. On the subject of PGs, we note that decorin (DCN) (see Section Fibril-associated proteoglycans), biglycan and fibronectin are some of the well-known PGs present in ECM; they are also known as members of the family of small leucine-rich repeat PGs (SLRPs). DCN is of interest in our discussion here because of its association with collagen fibrils; DCN can be found bound to the fibril surface. It is speculated that DCN may be able to bind to procollagen, i.e., before fibril formation. ${ }^{36,37}$ (Unfortunately, we still do not know where SLRPs co-traffick and interact with collagen along the secretary pathway.) Fibril-fibril fusion is thought to occur at regions along the fibril surface where there are no PGs. ${ }^{46,48}$ In principle, with a finite number of PGs present on the fibril surface (see Section Fibril-associated proteoglycans), this presents an opportunity for fusion of fibrils to could occur at DCN-absence sites. Under these circumstances, tip-to-tip fusion would involve the C-tip of the unipolar fibril. One could expect that the C-tip contains binding sites for N-tips of the other fibrils; these binding sites may also be energetically favourable for tipto-side fusion. ${ }^{47}$ Thus, this section has addressed the key processes by which collagen and collagen fibrils are synthesized. In the next section we discuss the structure of fibril-associated proteogylcans such as DCN and the role of these PGs in maintaining ECM organization.

\section{Fibril-Associated Proteoglycans}

\section{Locating Proteoglycans}

This section is concerned with the general structural features of the fibril-associated PGs. Typically, fibrilassociated PG contains a core protein bound to the fibril and one (or more) glycosaminoglycan (GAG) side-chain covalently bound to the SLRP core protein at one end while the other end extends into the PG-rich interfibrillar matrix (Fig. 3(A)). GAGs are linear chains of repeating disaccharides that occupy a large volume of space and exhibit strong swelling pressure at relatively low concentration. ${ }^{18,50}$ Fibril-associated PG GAG sidechain is predominantly anionic. ${ }^{18,50}$ In principle, owing to its large size and electronegative charge, this makes it unlikely for the GAG side-chain to fit within the fibril. ${ }^{10,11}$ In reality, they are constrained to extend outward from the fibril surface. ${ }^{10,11}$ Consequently, a stable antiparallel-running parallel to each other but with opposite alignments - noncovalent connection may be formed by any two overlapping GAGs on adjacent fibrils. ${ }^{18,50}$ GAGGAG interactions may involve the same type of PGs or even different types of PGs. ${ }^{50,51}$

Of great interest to ECM organization is DCN. DCN PGs are found on collagen fibrils at specific axial locations along the TC molecule. ${ }^{49}$ DCN PGs are thought to provide mechanical linkages between collagen fibrils. ${ }^{10,11,18,50}$ The binding site of DCN on the fibril surface is a contentious issue. DCN is thought to be located in the so-called $d$ (i.e., at $0.6 D$ ) and e (i.e., at $0.8 D$ ) band (with respect to a $D$ period of type I collagen) with respect to the axial staggered arrangement of TC molecules at the fibril surface. ${ }^{52}$ Where it is likely to be found would depend on the strength of the interaction of the TC molecule with $\mathrm{DCN}$, which is speculated to be different at the respective binding sites because of the different conformation and packing of the TC molecules on the fibril surface. ${ }^{52} \mathrm{Nev}$ ertheless, from a three-dimensional perspective (aided by three-dimensional electron tomography), it is believed that (1) the PG could adopt a six-fold arrangement around a collagen fibril - resulting in a pseudo-hexagonal or latticelike arrangement; (2) along the fibril, five to eight PGs 


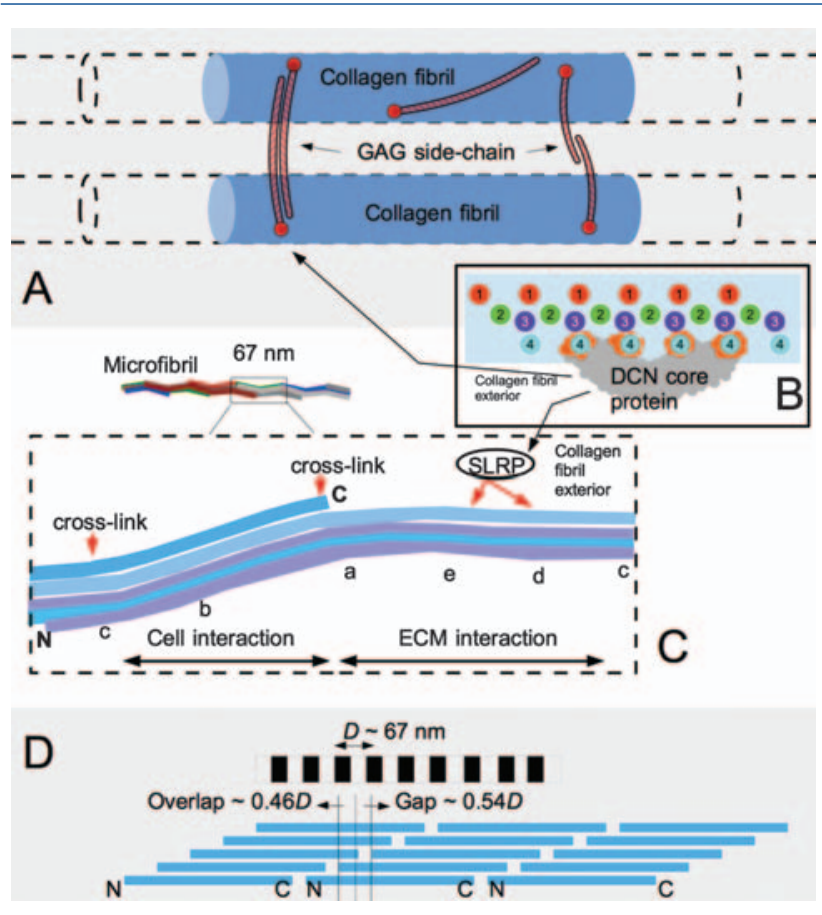

Figure 3. Interaction of decorin (DCN) proteoglycans (PGs) with collagen fibrils. (A) Schematic of glycosaminoglycan (GAG) side-chains on the DCN core protein of adjacent fibrils as mechanical bridges. (B) Schematic of the DCN core protein shape and the complementary collagen fibril surface (e band site) to describe the binding of the DCN to the fibril. The binding site occurs on the surface of the fibril, shown for the lateral packing structure of tropo-collagen (TC) molecules at $0.74 D$ (e band site) (C) Schematic of a microfibril showing the molecular segments of TC molecules. Indicated on the microfibril is the region describing the $D$ period. Inset shows the master control region encompassing the domains for cell interaction ( $a, b$ and $c$ bands) and ECM interaction ( $d$ and $e$ bands). (E) Hodge-Petruska organization for modeling the molecular arrangement along the fibril axis, where the organizational motif of the fibril comprises a group of five TC molecules. Inset is a schematic of a collagen fibril depicting the light and dark bands seen under an electron microscope; the schematic is intended to link the banding pattern to the gap/overlap regions in the Hodge-Petruska organization. Figures in panel (B) to (E) are adapted with permission from [10], J. P. R. O. Orgel, et al., Molecular and structural mapping of collagen fibril interactions. Connect. Tissue Res. 52, 2 (2011). ○ 2011.

per $D$ period could be found. ${ }^{50}$ These arrangements along and around a typical fibril have important implications on the regulation of the interfibrillar spacing. ${ }^{18,50}$ In the context of collagen fibrils providing reinforcement to ECM, the density of PGs could be a contributory factor to the mechanics of stress transfer from ECM to fibrils; this would be left to a later discussion (see Section Collagen fibril mechanics).

The locations of the PG at the $\mathrm{d}$ and e bands form part of a larger region-within the overlap region of the axial staggered arrangement of TC molecules-termed as the 'master control region' (MCR) (Fig. 3(C)). ${ }^{10,11}$ The $\mathrm{MCR}$ is thought to be able to accommodate cell interaction domains ( $\mathrm{a}, \mathrm{b}$ and $\mathrm{c}$ bands), and ECM functional domains (i.e., $\mathrm{d}$ and e bands), such as the intermolecular crosslink sites, the matrix metalloproteinase cleavage sequence, the fibronectin-binding site, and the triple-helix nucleation domain, to name a few. ${ }^{10,11,53}$ Interactions with the MCR could be facilitated by the presence of kinks or bends along the fibril because this would make "buried" sites accessible at the fibril surface. ${ }^{10,11,53}$

\section{Regulating Interfibrillar Spacing}

The GAG side-chain plays an important role in regulating the interfibrillar spacing. ${ }^{54,55}$ There are two types of GAG side-chains: keratan sulphate (KS) and chondroitin sulphate(CS)/dermatan sulphate(DS). CS/DS is attached to the core proteins of DCN and biglycan; KS is attached to the core proteins of lumican, keratocan and mimecan. ${ }^{18}$ Reaction with the amino sugars in the GAG chain yields a sulphate compound with a high negative charge at the unbounded end of the side-chain. The presence of the net negative charge will alter the state of hydration and the interactions with other PGs, and consequently affect the interfibrillar spacing between fibrils. ${ }^{18}$

Three-dimensional electron tomographic reconstruction of ECM reveals that PGs which form bridges demonstrate a tendency to bridge adjacent fibrils only tangentially, so enabling a PG chain to extend between more than two fibrils. ${ }^{18,50}$ Also, some PGs remain axially close to a single fibril. Nevertheless, these images suggest that the CS/DS stained filaments are relatively long $(\approx 300 \mathrm{~nm})$ and holds several fibrils together (bound near-tangentially). The simplest way to explain the bridges seen in the three-dimensional reconstruction is if each pair of GAG chains from adjacent fibrils is connected in an anti-parallel fashion. ${ }^{50,51}$ The anti-parallel associations would then be established by e.g., hydrogen bonds between the GAG chains and hydrophobic interactions. ${ }^{50,51}$ Of note, since the CS GAG contains more disaccharide motifs than KS GAG, this in turn presents more potential sites for sulphation and, consequently, yields more hydrophobic regions (see Section Locating proteoglycans)..$^{18,56}$ Since these antiparallel bridges are not covalent they can break and reconnect repeatedly. ${ }^{50}$ Owing to the high density of PGs, this makes it more likely for the formation of anti-parallel interactions between chains at different axial position on adjacent fibrils to occur. ${ }^{50}$ A theory of the reversible deformation of ECMs has been proposed to account for how the GAG chains forming anti-parallel associations can be broken and reformed with different overlapped lengths. ${ }^{50,51}$ According to this theory, the non-covalent GAG chain bonding reflects a fluid-like PG-rich interfibrillar matrix which serves to facilitate the transport of nutrients and molecules across ECM from the capillaries in the SCT. In principle, the fluid-like PG matrix also ensures that the relative position between collagen fibrils, as well as the interaction between the interfibrillar PGs, are not fixed. ${ }^{50}$ The fluid-like PG-rich interfibrillar matrix 
provides another perspective for modeling the process of PG dissociation and reconnection. Additionally, two complementary mechanisms, arising from thermal motion and osmotic pressure, have been proposed to explain the stability of the interaction between the connecting GAGs. ${ }^{18,50}$ These mechanisms are collectively termed as the Donnan effect. ${ }^{50}$ According to the Donnan effect, any two adjacent collagen fibrils experience an attractive force, arising from the thermal motion of the fibril-associated PGs, and a repulsive force, arising from osmosis. Thus the stability of the GAG-GAG interaction depends on the balance between the attractive and repulsive forces. ${ }^{18,50,54,55}$ Thermally-induced motion, which is responsible for collision between the biomacromolecules in ECM, could assist in ion transport, i.e., $\mathrm{Na}^{+}, \mathrm{K}^{+}$, in ECM towards the negatively charged ends of GAGs. ${ }^{57}$ Thus the build up of the positive ions around the GAG ends could screen the negatively charged GAGs, reducing the mutual repulsion between the GAGs. ${ }^{57}$ However, any increase in ionic concentration could also attracts water molecules by osmosis and this recreates an electrical imbalance around the interacting GAGs, ${ }^{18,50}$ leading to the disruption of the GAGGAG interaction. ${ }^{58}$ The Donnan effect complements the theory of reversible deformation of ECM to support to our understanding of how PG may readily dissociate and reconnect to enable flexibility in the interaction between collagen fibrils. Flexibility is also important here because it facilitates the passive movement of water and nutrients through the tissue. Of note, influx of water into the realm of collagen fibrils could lead to water gelation around the TC molecules and this could facilitate interactions (hydrogen bonding) between the water molecules and the 4hydroxyproline residues on the TC molecules. ${ }^{59}$

The regulation of the interfibrillar spacing is important in some SCTs such as cornea. ${ }^{54,55}$ From a design perspective the ordered assembly of fibrils in the corneal stroma makes good sense as it allows for light transmission; the opaqueness of other tissues such as tendon suggests that fewer PGs are present in these tissues than in the cornea. ${ }^{18}$ Of note, PG knockout mice results in collagen fibrils with irregular cross-sections and high variability in the cross-sectional size as well as a somewhat increased disorganized arrangement of fibrils within the tissue. ${ }^{48,60}$ Since these features are similar to those observed in older individuals (see TEM inset in Fig. 2(D)), it suggests that the density of fibril-associated PG decreases with age. ${ }^{61}$ However, this has yet to be confirmed.

Finally, although images from structural analysis have provided a basis for speculating on the role of DCN as the mechanical linkage that facilitates stress transfer between collagen fibrils, ${ }^{58,62}$ it is important to note that structural feature alone cannot indicate the mechanics of stress transfer via DCN for two reasons. First, we do not have precise knowledge of what type of bonds might exist between GAG side-chains. Second we do not know how other ECM biomacromolecules not identified yet may mediate GAG-GAG interactions between fibrils.

\section{Proteoglycan Core Protein Structure}

The argument for anti-parallel connection is only possible if each GAG were bound to a dimeric protein core of DCN. ${ }^{63}$ If the core protein is a dimer, it will be able to accommodate two opposing GAG side-chains and each of these side-chains will be able to connect to adjacent fibrils. Unfortunately, the dimerization concept is not widely accepted-whether DCN core protein is a dimer or monomer has been a subject of intense debate. ${ }^{10,11,63-65,67}$ Evidence for the monomeric model comes from rotary shadowing electron micrographs which reveal that the shape of the core protein appears as a curved bracket and is 'banana' shaped in the crystal structure. ${ }^{51,57}$ The monomeric argument is equally attractive because it readily lends to a resolution for how DCN core protein binds to the type I collagen fibril by interaction with at least four separate TC molecules. ${ }^{52,68}$ In this case, the four TC molecules are also members of four individual collagen microfibrils (see Section Microfibrils). ${ }^{52}$ The inner concave face comprises parallel beta strands that present a complementary fit for the fibril surface by facilitating the binding of the core protein to TC molecules. ${ }^{10,11,49,67}$ The energy for binding the core protein molecule with the fibril surface is estimated $\approx 170 \mathrm{~kJ} / \mathrm{mol}$; molecular modeling suggests that this interaction energy originates primarily from a hydrogen-bonding network established between the core protein concavity and four or more TC molecules. ${ }^{10,11}$ On the other hand, the dimer model presents a less favourable interaction energy with the collagen fibril surface as well as a less complementary fit to the fibril surface. ${ }^{10,11}$ Perhaps the key to this answer could be found in the biomechanics of collagen fibrils addressing the contribution of the shear action between the PG and TC molecule to the mechanism of stress transfer at the interface between the fibril and the PG-rich interfibrillar matrix. Alternatively, it is a timely reminder to recall that PGs play an important role in regulating, i.e., inhibiting, the lateral accretion of collagen fibrils. ${ }^{34,36,37,60,69}$ In this regards, perhaps more studies can be carried out to model fibril-fibril fusion that address the presence of DCN to help understand the implications of the structure of DCN core protein on the kinetics of fibril fusion and, base on the understanding gained, to infer the optimal shape of the core protein.

The discussion presented in these subsections on fibrilassociated PGs have summarized the well known findings concerning the location of the PG on the TC molecule of collagen fibril, the role of these PGs in maintaining the lateral spacing of collagen fibrils and the controversial structure of the DCN core protein. In the next section we explore the collagen based structural units beginning with those at the low dimensional levels, namely TC molecular 
structure (and how these aggregate into microfibrils) and the structure of microfibrils.

\section{Low Dimensional Structural Units \\ Tropo-Collagen Molecules}

In this paper, the term 'collagen' refers to the collagens that can self-assemble (axial staggering arrangement) to form fibrillar structures. The majority of the types of collagen that form the building blocks for fibrillar structures are categorized as types I, II and III. For the purpose of this discussion, the most common is type I. The reader is encouraged to refer to other sources for a more detailed account of the findings on collagen, ${ }^{1,29,33,70}$ particularly those derived from crystallographic and AFM studies. ${ }^{10,11}$

Several different polypeptide chains exist in ECM and these are denoted by the following 'alpha' symbols: $\alpha_{1}(\mathrm{I}), \alpha_{2}(\mathrm{I}), \alpha_{1}(\mathrm{II}), \alpha_{1}(\mathrm{III}), \alpha_{1}(\mathrm{~V}), \alpha_{2}(\mathrm{~V}), \alpha_{3}(\mathrm{~V})$, $\alpha_{1}(\mathrm{XI}), \alpha_{2}(\mathrm{XI})$ and $\alpha_{3}(\mathrm{XI})$. Thus, different combination of these polypeptide chains gives rise to different 'types' of collagen. ${ }^{1,29,33,70}$ Of note, type I collagen is characterized by a combination of two chains of $\alpha_{1}$ (I) and one chain of $\alpha_{2}$ (I); type II collagen contains three identical chains (i.e., $\left.\alpha_{1}(\mathrm{II})\right)$. Fundamentally, these different types of collagen differ in terms of the primary structure.

Extending from our discussion of the molecular motif in the helices of the TC molecule (Section Fibrillogenesis), we note that the most commonly found amino acid sequence is the Gly-Pro-Hyp triplet. ${ }^{59}$ A total of about 300 such triplets are arranged in an uninterrupted sequence to form a chain with short terminal domains at the end of the chain. ${ }^{1,29,33,70}$ (Note: the terminal domains do not exhibit the Gly-X-Y repeat structure.) While the different collagen types differ in terms of the physical and chemical characteristics, these differences manifest in the electrostatic and hydrophobic properties with consequences on the capacity for fibril assembly. ${ }^{15}$

The secondary structure of collagen comprises a central domain of $\alpha$-chains in a right-handed $\alpha$-helix along the axis of the central domain. ${ }^{10}$ The profile of the coiled structure is regulated by the (steric) repulsion between the Pro residue and Hyp residue. The bulk of the $\alpha$-helix is held together by peptide bonds but the side chains of amino acids are free from the influence of the peptide bonds. ${ }^{10}$ On average there are three residues per turn along the $\alpha$-helix-since every third residue of the (Gly-X-Y) ${ }_{n}$ domain is a glycine residue, this gives an appearance of a row of almost superimposed glycine residues on the surface of the $\alpha$ helix. ${ }^{1,29,33,70}$

From a design perspective, it is clear that the axial packing of TC molecules facilitates the self-assembly of fibrous units longer than the contour length $(\approx 300 \mathrm{~nm})$ of the TC molecule. ${ }^{10}$ Each TC molecule is staggered, connected by covalent cross-links, to adjacent ones by a multiple of $\sim 67$ $\mathrm{nm}$ according to the Hodge-Petruska scheme (Figs. 2(C), 3(D)). ${ }^{10}$ It follows that the whole fibril may be described by an organization comprising five TC molecules (i.e., the collagen fibril level 'motif'), and characterized by the overlap-gap repeat (which is commonly referred to as a $D$ period).

\section{Microfibrils}

In its simplest sense, a microfibril is made up of five subtly intertwined (staggered, when modelled from a one-dimensional perspective) TC molecules, with a righthanded twist. ${ }^{38,71}$ The diameter of the microfibril is $\approx 3.5 \mathrm{~nm}^{72}$ Microfibrils may be regarded as the basic structural units of the collagen fibril. ${ }^{71} \mathrm{~A}$ better understanding of the microfibrillar structure and its orientation in three-dimension can be derived by a careful analysisrunning over several $D$ periodic arrangements of 5 TC molecules-of the structural data derived from crystallographic studies. ${ }^{10,11}$ In relation to the structure of a collagen fibril, each TC molecule lying within the bulk of a fibril is surrounded by six neighbours, i.e., one in overlap and five in the gap regions (intermolecular spacing $\approx 1.3 \mathrm{~nm}) .{ }^{10}$ This leads to a quasi-hexagonal molecular packing arrangement that forms the characteristics crosssection of a microfibril. On the basis of these findings, including those from the recent AFM studies, it is proposed that the microfibril tilts is region-dependent: (1) in the overlap region, it tilts out of the fibril; (2) in the gap region, it tilts into the fibril. ${ }^{10,11}$

In addition to the tilts, neighbouring microfibrils also interdigitate with one another, connected by cross-links. Accordingly, each microfibril can probably accommodates a minimum of two to three interfibrillar (lysine-hydroxyllysine) cross-links; it is believed that there is a minimum of one intra-microfibrillar cross-links. ${ }^{71}$ Of note, if microfibrils slide more readily among themselves than the TC molecules within the individual microfibril ${ }^{73}$ this implies that there are fewer intermicrofibrillar cross-links as compared to intra-microfibrillar cross-links (see Section Microfibril sliding mechanics). To order of magnitude, we can identify the length of these cross-links with the intermolecular separation $(\sim 1 \mathrm{~nm}){ }^{71}$ The interdigitation comes about because the quasi-hexagonal packing of the collagen molecules-which continues throughout the collagen fibril-features neighboring $\mathrm{N}$-terminal and C-terminal containing molecular segments that are contained within neighboring microfibrils, instead of embedding into the microfibril. Owing to this arrangement, microfibrils have yet to be isolated because they are physically not separable structural units. ${ }^{38,71}$ Additionally, we note that the disruption of the $\mathrm{N}$ - and C-terminal bonds during collagen extraction affects the structure of the collagen fibril as well as the microfibril, hence the difficulty in isolating microfibrils. $^{71}$

The discussion presented in these subsections have highlighted the well-known findings concerning TC molecular structure, how the TC molecules aggregate into microfibrils, and the recent speculations addressing the organization of micofibrils in collagen fibrils. In the next section, 
we explore the findings on collagen fibrils, particular the different models used in understanding the structure of collagen fibrils, namely the axial straggered TC molecules, the microfibrillar argument for the basic structural unit as framed within the context of collagen fibril and the controversial subject on the collagen fibril length.

\section{Collagen Fibrils}

Analysis of X-ray diffraction (XRD) patterns of collagen fibrils reveals that the different reflections in two regions known as the equatorial and meridional (Fig. 5(A)) regions indicate a state of anisotropy. ${ }^{39}$ (In contrast, the diffraction pattern of an isotropic system such as randomly dispersed crystalline powders is described by circular reflections centred at the beam centre.) The meridional region reveals a series of regularly spaced Bragg reflections, which is key to understanding the crystalline (axially staggered) arrangement of TC molecules. ${ }^{39,74,75}$ We note that the axial staggering of five (repeating) TC molecules (Figs. 2(C) and $3(\mathrm{D})$ ) is a one-dimensional model of collagen fibril that is used to explain the $D$ bands characterising the distinctive gap and overlap regions that are commonly seen in electron micrographs. Changes in the reflections (broadening, position) are attributed to changes in the long-range axial crystallinity (i.e., the gap and overlap regions of the $D$ repeat) of the semi-crystalline structure of the fibril and this would influence the mechanical properties in the axial direction of the fibril.

Evidence from images of fibrils derived from threedimensional electron tomographic reconstruction ${ }^{76}$ has suggested that Type I collagen fibrils are constructed from microfibrils. ${ }^{77}$ These images reveal that the crosssection of an individual (thin) fibril features a core of four microfibrils surrounded by a ring of ten microfibrils (known as the $10+4$ microfibrillar arrangement). Analysis of the axial mass distribution measurements reveals that each microfibril contains five collagen molecules in crosssection and this corroborates the predictions from X-ray crystallographic studies. ${ }^{71}$

The profile of collagen fibrils has been a contentious issue. Findings from developing SCTs indicate that the ends of the collagen fibrils are tapered. ${ }^{31,44,78,79}$ Taper is also found in some mature SCTs ${ }^{80-82}$ Isolated collagen fibrils from the medial collateral knee ligaments of mature rats are found to have diameters of 40-109 nm, lengths of $12-30 \mu \mathrm{m}$ and aspect ratios of $550-625 .{ }^{81}$ Collagen fibrils from the spine ligaments of sea urchin are slenderer than those from the rat tissues; these sea urchin collagen fibrils have a mean diameter $\approx 66 \mathrm{~nm}$, a mean length of $\approx 225 \mu \mathrm{m}$ and aspect ratios of 2250-3300..$^{80}$ Additionally, the analysis presented here can also be explained by extending the Hodge-Petruska onedimensional model of the axial staggering of TC molecules in three-dimensional space to help reconstruct models of fibril with tapered ends. ${ }^{83}$ Nevertheless, because the interfibrillar bonds (mediated by PGs) increases with age, this presents difficulty in isolating collagen fibrils from SCT in old individuals. Thus, the investigation on the profile of isolated fibrils has been limited to SCTs from developing individuals. ${ }^{81}$ Taper is not confined to native collagen fibrils in the tissues; it is also exhibited in Type I collagen fibril synthesized in vitro. ${ }^{41,83}$ TEM studies reveal that the reconstituted collagen fibril segment is asymmetric, characterizes by a long and a short tapered end, possibly due to different accretion (the rate of mass uptake per unit area). Regression analysis of the axial mass distribution versus distance along the fibril suggests that the profile of the taper is somewhat paraboloidal. ${ }^{41,44,82}$ In all cases, owing to the possibility of distorting the individual fibrils during specimens preparation for imaging, we suspect that the fibril profile could lie somewhat between an ellipsoidal and a conical profile. The shape of fibrils is an important subject because it is key to understanding the nucleation, growth and remodeling of collagen fibril. ${ }^{43}$ Earlier findings have suggested that accretion is inversely proportional to the diameter. ${ }^{41}$ Thus, accretion is greatest at near the tip but lessens away from the tip. ${ }^{41,43}$ Latter findings corroborate this prediction; in other words, fibrils can grow at the tips and centre in a coordinated manner. ${ }^{44,82}$ Nevertheless, the intriguing fibril shape has raised a series of questions that remain unanswered. Are there more fibrils with tapered ends in tissues of developing and young individuals than in tissues of mature and old individuals? Conversely, do uniform cylindrical fibrils predominate in the tissues of mature and old individuals? Although we may not have the answers to these questions for now, the fibril profile presents interesting challenges for how fibril provide reinforcement to ECM and this is left to a later discussion (see Section Collagen fibril mechanics).

Another point that we would like to emphasize concerning fibril shape is the implication on fibrillogenesis (see Section Fibrillogenesis). For the relevant cells to play an important role in the macroscopic assembly of ECM, e.g., fibrillogenesis, the intermediates formed by the cell must be of a manageable size and shape. Early fibril segments would be sufficiently small to allow rearrangement by the cell by juggling or cradling or pushing the segments into position within fibripositors; the tension exerted by the cell could also aid in rearrangement the short segments. ${ }^{36,37}$ As the fibril grows into longer segments, with one end in the fibripositor and the other end in ECM, it is likely that the directional alignment of the latter end in ECM would be influenced by the collagen fibrils in the vicinity. ${ }^{36,37}$

This section ends our discussion on the structure of collagen fibrils. In the next section, we explore the findings on collagen fibres and the larger issues addressing collagen fibres in the context of microscopic crimps and the insertion sites at the tissue junctions. 


\section{Collagen Fibres}

\section{Microscopic Crimps}

The microscopic length scale of ECM is associated with collagen fibres. Collagen fibres are observed in aggregates (bundles); a bundle of these fibres constitute a fascicle. In terms of the collagen fibres, SCTs such as tendons and ligaments share very similar structure, ${ }^{20}$ revealing highly paralleled bundles collagen fibres, with fibre diameter on order of magnitude of $100 \mu \mathrm{m}$ (Fig. 1(B)). ${ }^{84}$ On further examination, three key features emerged:

(1) a cross-connection of cells between these fibres; ${ }^{84}$

(2) an organized arrangement of fibres into fascicles; ${ }^{84}$

(3) microscopic crimps on the fibres.

The crimps of collagen fibres have been attributed to a helical twisting of the individual collagen fibres, with a predetermined amplitude and wavelength, which combine to form a three-dimensional (twisted) rope-like structure. ${ }^{7}$ Nevertheless, the waveform disappears when the tissue is stretched; as we would expect, the waveform reappears when the tissue is unloaded. This structural appearance and disappearance behavior is reversible as long as the strain is confined to a small strain range, e.g., 0.04-0.05. , $^{8,9}$ This small strain region corresponds to the toe-to-heel region on the stress-strain curve. In addition, some fibres could also pass (i.e., interweave) from one fascicle to another. ${ }^{84}$ It is thought that the interweaving of collagen fibres between fascicles facilitates a more uniform distribution of the force (generated by the muscle cells) over the whole area of insertion at the muscle-tendon junction (MTJ). ${ }^{84}$

On the same length scale as collagen fibre bundles, one can also find cells (e.g., tenocytes in tendon) lining the collagen fibres. ${ }^{8,9,85,86}$ Each cell is assumed to be attached to a fibre. ${ }^{87}$ Each of these cells contains a long nucleus and possesses long cell processes that interdigitate among cells along the same fibre as well as those on adjacent fibres; ${ }^{8,9,85,86}$ these processes facilitate cell-cell communication. ${ }^{88}$ We shall highlight the fibre-associated cells in regards to their usefulness as strain markers for monitoring fibre extension, fibre-fibre sliding and mechanotransduction in a later discussion (see Section Collagen fibre sliding mechanics).

Although three-dimensional magnetic resonance images have yielded remarkable structural details about ligaments and tendons that are useful for clinical diagnosis as well as for the development of more realistic computer models of the tissue for understanding the structure-function relationship, ${ }^{89-91}$ the issue concerning whether collagen fibres span the entire length of the tissue has yet to be resolved. ${ }^{92}$ The collagen fibre length issue is important because it relates to the mechanics of tissue extension ${ }^{93}$ as well as to tissue growth. ${ }^{94}$ Evidence from measurements of fibre-associated cell undergoing displacement during uniaxial extension of collagen fibre suggests that fibre-fibre sliding is possible provided the fibres are short relative to the whole tissue..$^{8,9,85,86}$ Alternatively, fibre-fibre sliding may also be possible if these fibres span the midsection of the tissue but only a proportion would terminate at the junction connecting to another tissue. ${ }^{93}$

\section{Tissue Junctions}

The junction connecting a SCT, e.g., ligament or tendon, to the bones is an important region where reconstruction surgery is concerned because this is where high stresses may develop during extreme loading and consequently fracture is likely to take place here. ${ }^{95}$ The insertion sites are called enthesis. ${ }^{96}$ Tendons or ligaments connect to bone via the periosteum. ${ }^{96}$ No discernible boundary has been observed at the enthesis so far. ${ }^{96} \mathrm{SCT}$ is oriented obliquely to the insertion site before it enters the bone; this means that the SCT would have been in contact with the bone before it enters the attachment site. This region of contact features dense fibrous tissue that is identified with bundles of collagen fibres; the dense fibrous tissue is thought to be continuous with the rest of the tissues. ${ }^{96}$ From an engineering perspective, this dense fibrous tissue could help to spread the load over a wider area which would otherwise lead to high stress concentration. ${ }^{97}$ At the entrance into the insertion site, the SCT features a flare-comprising less densely arranged bundles of collagen fibres-which covers a large area over the bone surface. Inside the insertion site, as the distance increases, it is observed that the collagen fibres becomes denser and undistinguishable with the rest of the matrix. ${ }^{97}$

The other junction of interest is the MTJ (see Section Microscopic crimps). The key features in the MTJ are a series of terminal extensions at the ends of muscle cells. ${ }^{98,99}$ Microscopic examination of these extensions reveals an array of thin myofilaments inserted into periodic sub-sarcolemmal densities underlying the cell membrane and its external lamina. ${ }^{98,99}$ These cytoplasmic extensions are villous processes which can extend into ECM of the adjacent tendon. ${ }^{98,99}$ These villous processes resemble an extensive network of cylindrical folds that interweave with one another. ${ }^{3}$ Structurally, these villous processes yield a high surface area (to volume ratio) at the MTJ, which can help to reduce the stress between these two tissues during force transmission. ${ }^{3}$ It is straightforward to see that as the individual muscle cells grow larger, the cross-sectional area and surface area of these cells would also increase; ECM around the muscle cells must also increase in proportion to the increased surface areas of the muscle cells. ${ }^{3}$ In principle, since larger muscle cross-sectional area leads to larger force generated, the tendon that transmits the force must possess a sufficiently large cross-sectional area to be able to accommodate the force. Otherwise the tendon would be subjected to high stresses and this would increase the risk of rupture when the stress reaches the rupture stress of the tendon. ${ }^{3}$ It is interesting to note that the muscle cells in the MTJ are somewhat regularly spaced with a preferred orientation in the direction of 
the surrounding collagen fibres. ${ }^{3}$ The structural orientation of the cells could be influenced by the orientation of the collagen fibres, which provide the structural support to these cells. ${ }^{100}$ The age-related loss of skeletal muscle mass (as well as function) is linked to apoptosis and this is widely believed to occur in muscle cells. ${ }^{24}$ However, recent evidence from experiments suggests that muscle cells represent a small proportion of the apoptosis in ageing muscles. ${ }^{24}$ Instead an appreciable proportion of cell death comes from the satellite (capillary) cells of the collageneous tissue around the muscle cells. ${ }^{24}$ One immediate and important implication is that it could lead to impairment of capillary functions, i.e., reducing their capacity to deliver nutrients and oxygen and to remove toxic metabolic products. This could have grave repercussions, extending from the muscle to ECM of MTJ, leading to decrease mass in the structural units at the low dimensional levels of ECM, with deleterious effects on ECM functionality. ${ }^{24}$

Before we wrap up the discussion of structure, we would like to clarify that the term collagen fibres should not be confused with elastic fibres, which is a term given to another type of insoluble structures that predominates ECM of dynamic tissues such as blood vessels, skin and the lungs. ${ }^{101}$ These elastic fibres are composed of elastin (a cross-linked core) and (an outer layer of) fibrillin microfibrils. ${ }^{102}$ Of note, elastin confers the ability of elasticity to these tissues. This means that the tissues are efficient at absorbing and storing mechanical energy (derived from deforming these dynamic tissues under physiological forces) as well as releasing the energy to drive passive recoil (contraction) when the load is released, thus enabling the regulation of the blood pressure. ${ }^{101,103}$ It is also thought that elastin and the elastic-fibre related proteins are found in the intrafascicular matrix to provide elasticity. ${ }^{104,105}$ For simplicity, elastic fibres may be considered as a biological example of fibre composite in which the fibrillin microfibrils provide reinforcement to the elastin core. In particular, these two components perform distinct roles; elastin stores energy and drives passive recoil, whilst fibrillin microfibrils direct elastogenesis, mediate cell signalling, maintain tissue homeostasis via TGF- $\beta$ sequestration and potentially act to reinforce the elastic fibre. ${ }^{101}$ Further details regarding the dynamics of these elastic tissues are found elsewhere. ${ }^{101}$

\section{Summary}

This section summarizes the discussion on the features of ECM structural units of the respective levels of the hierarchical architecture.

(1) TC molecules may be regarded as the structural units of the lowest level. Moving up the hierarchy and in the order of increasing level, we have microfibril, collagen fibril, collagen fibre, fascicle and finally the whole tissue.
(2) Following the synthesis of collagen, an entropic-driven self-assembly process regulating the aggregation of TC molecules into early collagen fibrils takes place in fibripositors. Thereafter the collagen fibrils grow by fusion with one another

(3) Fibril-associated PGs such as DCN are responsible for maintaining the organization of ECM, namely interfibrillar spacing.

(4) The triple (right-handed) helices of collagen molecule, known as the TC molecule, are held together by peptide bonds; these helices possess the necessary hydrophobic and electrostatic properties possibly to facilitate the process of collagen fibril self-assembly.

(5) Microfibrils are the basic fibrillar units of ECM. A microfibril is made up of five intertwined TC molecules arrange in a right-handed twist (from a cross-sectional perspective). The axial arrangement of the microfibrils along the entire length of the collagen fibril involves a series of interdigitation.

(6) Thus, collagen fibrils are made up of microfibrils. Collagen fibrils are long and slender, exhibiting tapered ends in ECM of developing tissues. In mature tissues, uniform cylindrical fibrils predominate in ECM.

(7) Collagen fibres as well as bundles of collagen fibres (otherwise known as fascicles) are the structural units of the upper levels. These units are characterized by microscopic wavy patterns with cells lining the fibres. Collagen fibres are involved in anchoring the SCT to other tissues, e.g., bone (as in bone-ligament-bone complex or tendonbone complex) and muscles (as in MTJ). Whether the fibres span the entire length of the tissue or a portion of the tissue length is not well understood but this has important implications concerning our understanding of how the tissue extends under load and how the tissue grows with age.

\section{EXTRACELLULAR MATRIX (ECM) FUNCTION Mechanical Response Graphs}

In the study of the strength of materials, the concept of continuum underpins our understanding of how a material responds to an external applied force. The continuum mechanics concept is applied to structural engineering to understand how different structures interact with one another under an external applied force. From a biological perspective, the key question that a tissue engineer faces that relates to the biomechanics of SCT is: does ECM behave as a continuum or as discrete structures? Strictly speaking, the answer would depend on the relevant biological issues and the length scales involved. In order to model complex systems such as SCT, we would have to make simplifying assumptions - a model of ECM has to be sufficiently simple to enable the biological question to be answered-it is not good practice to base an investigation on an over elaborate model. Also, the simplicity, or otherwise, of the method of analysis should not 
be a measure of the scientific worth of an investigation into ECM. Thus, it is valid to consider ECM as a continuum only if (i) it is dramatically larger than the dimension of the smallest structure identified and (ii) one is interested only in deformations on the whole tissue level, even with the knowledge that ECM components such as collagen fibrils have very different structure and properties from the surrounding matrix in which the fibrils are embedded.

In this section, we are concerned with the mechanics of the structural units of ECM at the respective level of the hierarchical architecture. In principle, to understand the contribution from ECM components associated with the respective level of the hierarchical architecture to the mechanical property of the SCT, a set of mechanical response curves such as the stress versus strain curves at each level is desired. ${ }^{106,107}$ To this end, the discussion that follows will address the basis of stress uptake by the respective structural units at the different stages of the loading process-framed in the context of the stress-strain curve for a SCT undergoing uniaxial extension (see justifications in Section Introduction).

A sketch of a typical stress-strain relationship is illustrated in Figure 4(A) for a tendon, with sequential images to depict how it is recruited into tension and how it eventually ruptures. The basic features associated with the following regions, namely toe-to-heel, linear, transition (peak stress), and failure, is also found in ligaments. The stressstrain relationship is derived from the load-displacement data. An example of the load-displacement relationship is shown in Figure 4(B) for anterior cruciate ligaments tested intact in the femur and tibia (these were used for gripping during the test). ${ }^{108,109}$ With reference to Figure 4(B), the graph in the left panel corresponds to a displacement rate of $10 \mathrm{~mm} / \mathrm{min}$ (intended for modeling normal physiological loading); the graph in the right panel corresponds to a displacement rate of $500 \mathrm{~mm} / \mathrm{min}$ (intended for
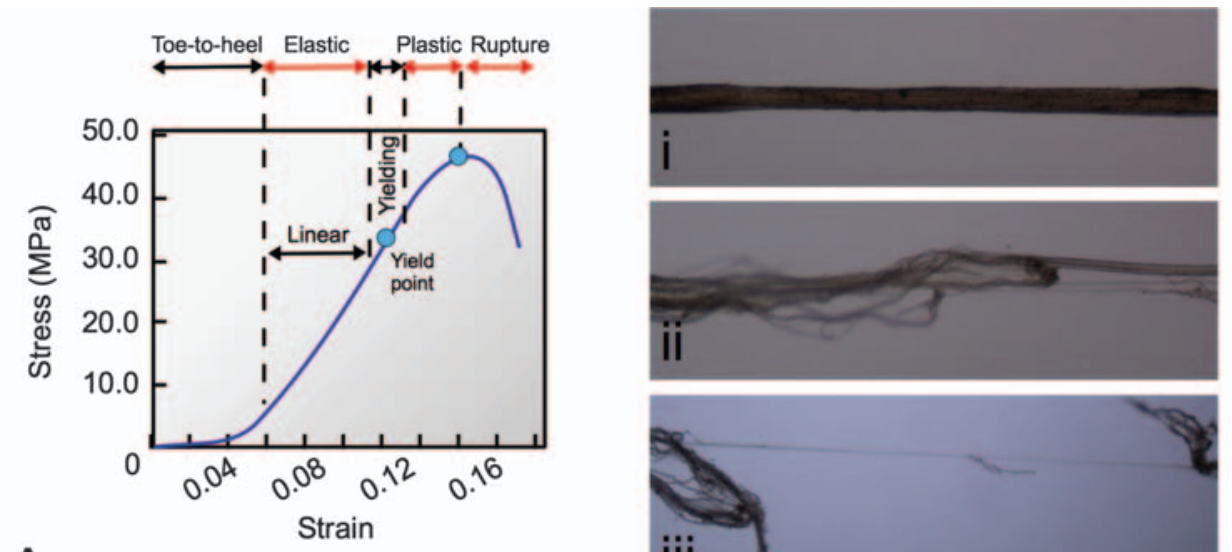

A
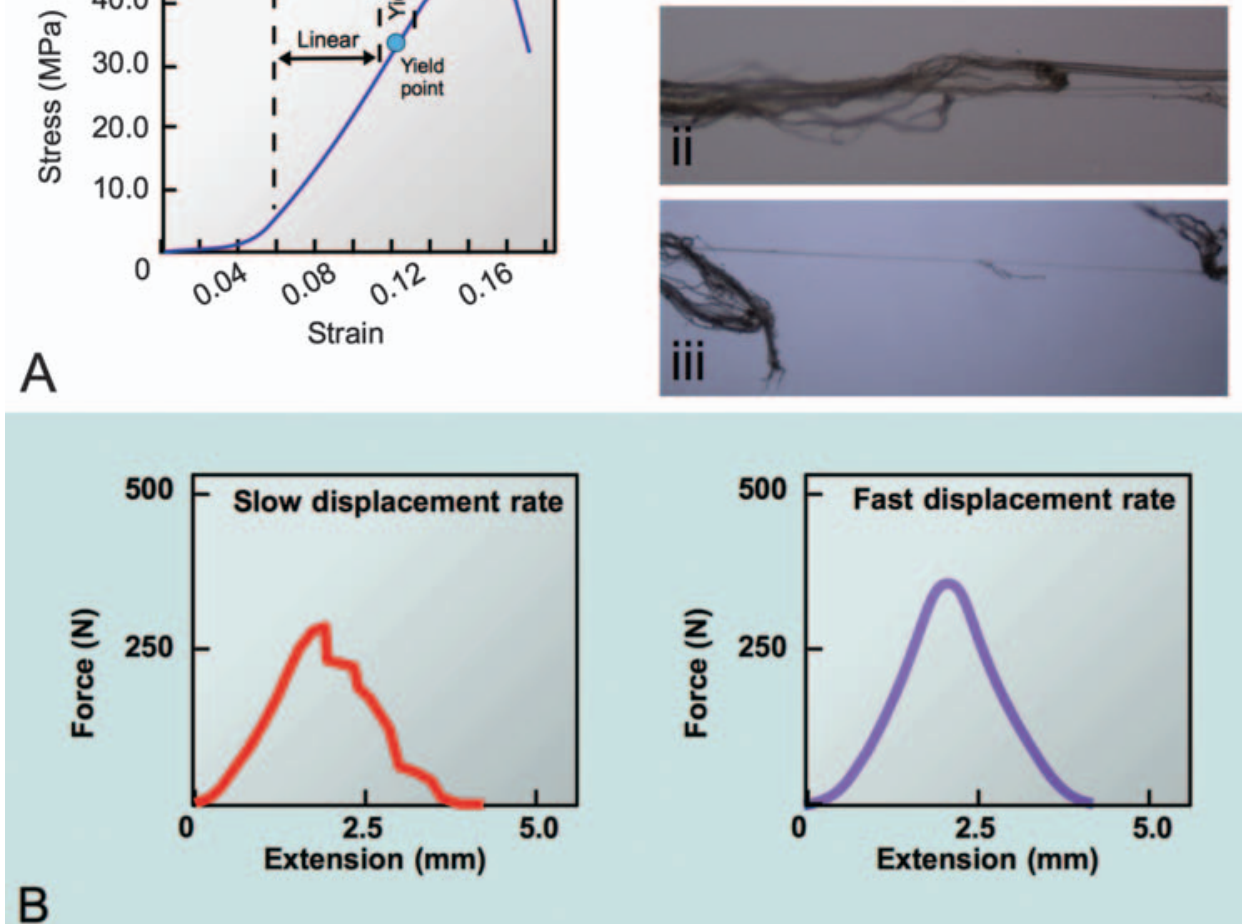

B

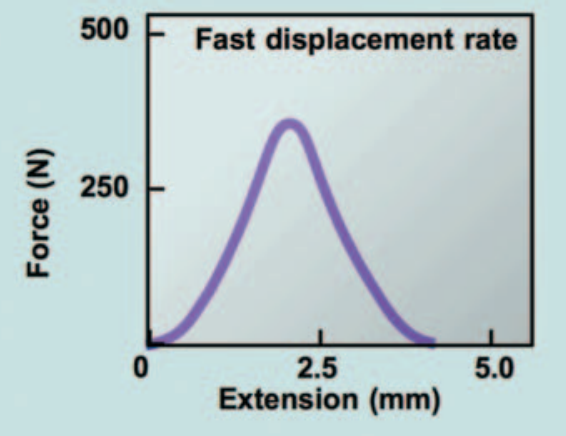

Figure 4. The mechanical response of soft connective tissues to an external load. (A) A sketch of the graph of stress versus strain for a hydrated tendon fascicle (rat tail). Displacement rate, $5 \mathrm{~mm} / \mathrm{min}$. Reprinted with permission from [8], H. R. C. Screen, et al., Local strain measurement within tendon. Strain 40, 157 (2004). $\odot$ 2004, John Wiley and Sons. Hydrated tendon fascicle (i) at initial loading, (ii) defibrillating immediately after the maximum stress and (iii) rupturing to the point where the ruptured ends were bridged by only a single strand of collagen fibre. (B) A sketch of the graph of force versus displacement for a boneligament-bone complex corresponding to a slow displacement rate $(10 \mathrm{~mm} / \mathrm{min})$ (left panel); the sketch to the right corresponds to a faster rate (50 times that of left panel). Figures in panel (B) are adapted with permission from [108], G. Azangwe, et al., Macro and microscopic examination of the ruptured surfaces of anterior cruciate ligaments of rabbits. J. Bone. Joint Surg. 82-B, 450 (2000). () 2000. 
modelling extreme loading). The profiles are not quite the same because the basic mechanisms that predominate in the slower rate and the faster rate may not be same. ${ }^{108,109}$ For example, at high displacement rates, the mechanisms regulating fibril rupture predominate. High displacement rates are associated with high stiffness and high strength; low displacement rates are associated with low stiffness, and low strength. ${ }^{108,109}$ The reason for these differences is that the mechanical response of SCT is time-dependent and the overall mechanical response is a combination of both the elastic response and the viscoelastic responses. ${ }^{110}$ In particular, the viscoelastic behaviour can be investigated by creep tests and stress relaxation tests. ${ }^{11}$ It is wellknown that the mechanical response associated with the creep process involves increases in strain with time when the tissue subjected to a constant load; ${ }^{112}$ the mechanical response associated the stress relaxation describes an increase in stress when a fixed strain is first introduced, followed by a decreasing stress with time. ${ }^{113}$ From the perspective of uniaxial extension, the mechanical responses (i.e., stress-strain curve to rupture, creep and stress relaxation) share similar underlying mechanisms from molecular level to fibre level. Examples of these mechanisms are the time dependent rearrangements of the collagen components within the loosely ordered and hydrated PG complexes, and concomitant interdiffusion of water molecules from one nanoscale compartment to another, or between levels of the hierarchical architecture. ${ }^{111}$ Of note, in most tensile test experiments reported so far, the time-scales associated with these loading tests, e.g., $30 \mathrm{~min}$ for a complete run using X-ray crystallographic approach ${ }^{106,107}$ or 1 to $2 \mathrm{~min}$ at $0.06 \mathrm{~mm} / \mathrm{sec}$ for micromechanical testing of fascicles, ${ }^{27}$ are comparable to the relaxation time (200 to $300 \mathrm{~s}$ ) of the $\mathrm{SCT}^{111}$ or even much longer than the creep time ( 2 to $4 \mathrm{~ns}$ ) of the TC molecule. ${ }^{114}$ By establishing the discussion in the context of the stress-strain curve (e.g., in uniaxial extension) derived from these experiments, this provides for further generalization of insights that can enable predictions to be made on the viscoelastic effects.

\section{Tropo-Collagen Molecular Mechanics Molecular Force-Displacement Curve}

When a tendon or ligament undergoes uniaxial extension, the various structural units which made up the tissue would also be stretched, internally, throughout all the parts and in due proportion, down to a very fine scale, i.e., down to the TC molecular level. In general, depending on the magnitude of the load acting on ECM biomacromolecules, e.g., TC molecules, there are four general modes of molecular deformation: (i) domain motion, (ii) domain deformation, (iii) domain unfolding and (iv) denaturing of secondary structures such as the alpha-helices in a TC molecule. ${ }^{100,115-118}$ For an illustration of each of these modes see Figure 5(B). Domain motion, which is thought to occur at loads ranging from $1-10 \mathrm{pN}$, is predominated by the loops and turns that join the domains together. ${ }^{100}$ When the load on the molecule increases further (e.g., tensile loads ranging from $10-100 \mathrm{pN})$, the domain may begin to elongate. ${ }^{100}$ At larger loads $(>100 \mathrm{pN})$, domain unfolding may occur. ${ }^{100}$ At even larger loads $(\gg 100 \mathrm{pN})$, the triple helix is disrupted or ruptured. ${ }^{100}$ TC molecules are connected by a mixture of covalent cross-links, van Der Waals bonds and hydrogen bonds. These connections arise from the forces of interaction between the lysine or hydroxyl-lysine amino residues at the N-terminal and C-terminal telopeptides and the lysine or hydroxyl-lysine amino residues within the bulk of the telopeptides. ${ }^{1}$ These forces of interaction enable the TC molecule to take up stress and transfer it to the adjacent ones. ${ }^{106,107}$

A sketch of the graph of tensile force versus extension of a TC molecule undergoing uniaxial extension, derived from an AFM study is illustrated in Figure 5(C). ${ }^{16}$ Inset in Figure 5(C) shows a graph of force versus extension derived from MD simulation. ${ }^{119}$ Of note, the force-extension relationship from the experimental and simulation study shares similar features (marked by labels $\mathrm{a}, \mathrm{b}$ and $\mathrm{c}$ on the curves), namely a toe-to-heel region (up to point a), a gradual rise in force with increase in extension (point $a$ to $b$ ) and a rapid increase in force with increase in extension (point $b$ to $c$ ). In particular, the simulation modeled the stretching of a short segment (initial length $\approx 8 \mathrm{~nm}$ ) of the TC molecule, in a physiological environment (i.e., aqueous saline solution of sodium chloride). For this reason, the maximum extension of the TC molecule (contour length $\approx 310 \mathrm{~nm}$ ) derived from the experimental study was two orders of magnitude larger than that predicted by the model. Interactions between chemically bonded neighbours (by allowing for bond stretching, angle bending, dihedral and improper dihedral changes) and interactions beyond nearest neighbours (Lennard-Jones and Coulomb potential) were implemented to model the molecular response of the molecule to the external load. We highlight four main points from this comparative analysis as follows.

(i) The initial loading from the origin up to point a involves the straightening of slacks within the TC molecule. The molecular process addressing the straightening of slacks ensures that this would result in an increase in the length of the gap region with respect to the overall length of the overlap region. ${ }^{120}$ Thereafter, the bonds experience loads that cause them to deform in tension; bonds not parallel to the axis of the TC molecule will be aligned (by angular stretching) into this direction. ${ }^{119}$ Since the tensile force could act on the three strands of the TC molecules simultaneously, thus the applied force could increase by a factor of three. Hence this leads to the jump in the force generated in the TC molecule (Fig. 5(C)).

(ii) The region from point $a$ to $b$ is attributed to the unwinding of the triple helix. ${ }^{16,122}$ As the triple helix of the TC molecule unwinds, this changes the dihedral angles 

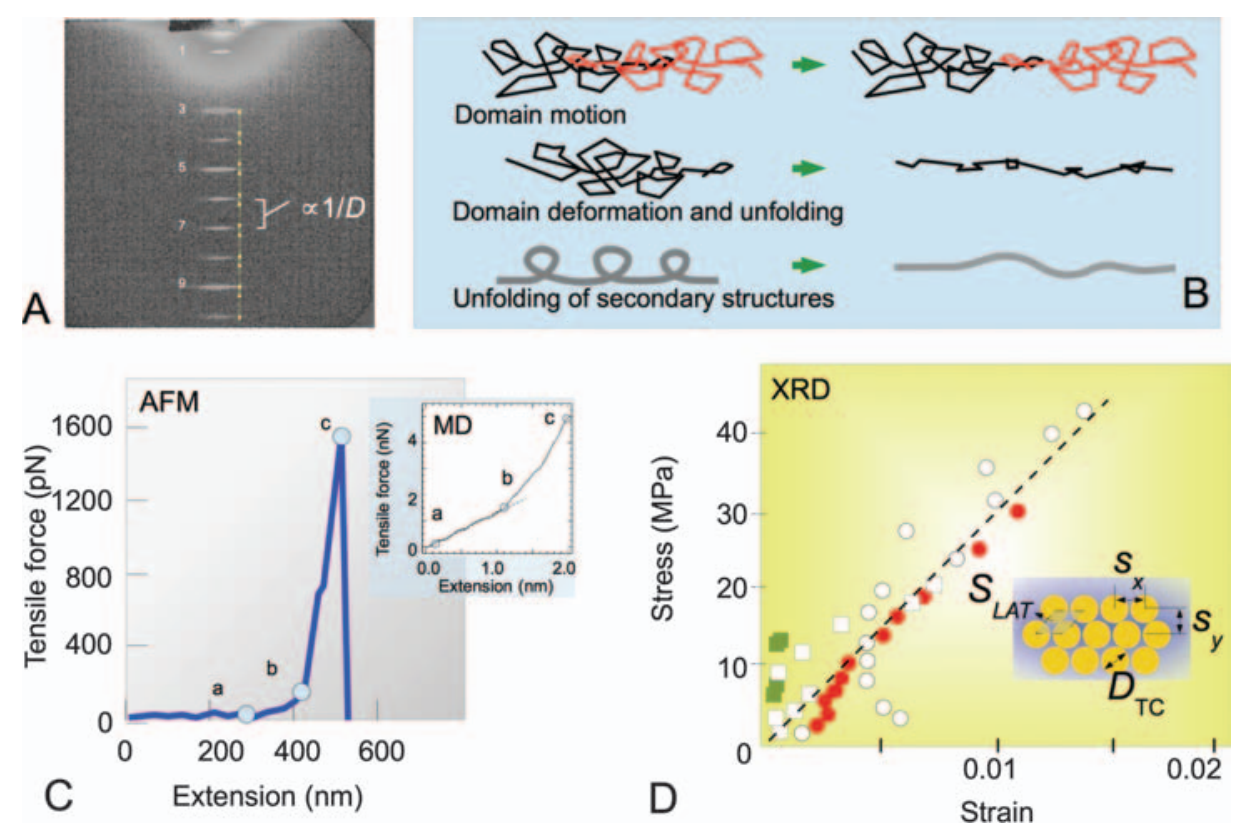

Figure 5. Mechanics of tropo-collagen (TC) molecules. (A) Typical X-ray diffraction (XRD) pattern showing the meridional reflections of tail tendons from a 1.6 weeks old mice. The regular spacing between the reflections is $\propto 1 / D$, where $D(\approx 67 \mathrm{~nm})$ is the $D$ period. Unpublished data, acquired at the Daresbury Laboratory (UK, Beamline 2.1). (B) Protein deformation illustrated for domain motion, domain deformation and unfolding, unfolding of secondary structures. Adapted with permission from [100], C. Zhu, et al., Cell mechanics: Mechanical response, cell adhesion, and molecular deformation. Annu. Rev. Biomed. Eng. 2, 189 (2000), $\odot$ 2000. (C) Graph of force versus displacement for a (Type I collagen) TC molecule undergoing uniaxial extension, based on results derived from stretching the molecule by atomic force microscopy (AFM). Reprinted with permission from [16], L. Bozec and M. Horton. Topography and mechanical properties of single molecules of type I collagen using atomic force microscopy. Biophys. J. 88, 4223 (2005). @ 2005, Elsevier. Inset is a sketch of a similar graph but derived from molecular dynamics (MD) simulation. Reprinted with permission from [119], P. J. in't Veld and M. J. Stevens, Simulation of the mechanical strength of a single collagen molecule. Biophys. J. 95, 33 (2008). $\odot$ 2008, Elsevier. (D) Graph of stress versus strain of TC molecule derived from the XRD of five dehydrated (bovine) Achilles tendons subjected to tensile loads. Reprinted with permission from [106], N. Sasaki and S. Odajima, Stress-strain curve and Young's modulus of a collagen molecule as determined by the X-ray diffraction technique. J. Biomech. 29, 655 (1996). @ 1996, Elsevier. Inset is a model of the transverse (2D quasi-hexagonal) packing of TC molecules in a dehydrated tendon. Circles represent the cross-section of the molecules, with diameter $D_{\mathrm{TC}}$. Symbol $\mathrm{S}_{\mathrm{LAT}}$ represents the area of a parallelogram unit cell; $s_{y}$ and $s_{x}$ are crystallographic parameters.

in the backbone region of the molecule. ${ }^{119}$ It turns out that a force $\approx 200 \mathrm{pN}$ is needed to cause the molecule to unwind. ${ }^{119}$

(iii) The unwinding effect may continue into the steep region (b to c), with a mixture of bond and angle stretching in the backbone. ${ }^{119}$

(iv) Within the margin of experimental errors, we note that the forces from the AFM study are in good order of magnitude agreement with the predicted forces from the MD study.

Interestingly, the above findings from MD study of the uniaxial extension of a TC molecule corroborate the results from uniaxial extension experiments complemented by Raman spectroscopy. In particular, Raman spectroscopy reveals that the toe-to-heel region corresponds to the straightening of molecular kinks. Accordingly since the Raman wavenumber of $822 \mathrm{~cm}^{-1}$ (which is associated with backbone vibration) reveals no appreciable shift, it is speculated that this kink straightening process yields little stress uptake in the structural unit. ${ }^{19,121}$ Raman spectroscopy also reveals that beyond the toe-to-heel region, the TC molecules exhibit a combination of stretching and sliding action; these are reflected in the shift (to a lower value) in the Raman wavenumber corresponding to the stretching of the collagen $(\mathrm{C}-\mathrm{C})$ backbone. ${ }^{19,121}$ This shift continues in the same direction (i.e., to a lower value) with increasing applied strain up to a strain of $0.13 .{ }^{19,121}$ Beyond the strain of 0.13 , the shift is directed towards a higher value; this may suggest that yielding has occurred at the molecular level, possibly a prelude to rupture of bonds. ${ }^{19,} 121$

The MD study has demonstrated that a TC molecule could extend beyond the length of the TC molecule. ${ }^{119}$ In particular, a persistence length $L_{P L} \approx 14.5 \mathrm{~nm}$ (see Section Tropo-collagen mechanical properties) could yield a strain of $\approx 0.14 .{ }^{14}$ These strain findings are consistent with those derive from X-ray crystallographic studies. ${ }^{106,107}$ Comparison with the results from X-ray crystallographic studies is important because it provides deeper insights into the origin of strain, implicating how 
the distance between neighbouring amino acids along the helical axis of the TC molecule changes with respect to the overall tissue extension. Figure 5(D) illustrates a plot of the stress-strain curve of TC molecule derived from the X-ray crystallographic study (i.e., simultaneous mechanical testing and XRD). Here the strain in a TC molecule is given by

$$
\varepsilon_{\mathrm{TC}}=\left[w_{\mathrm{XRD}}-w_{\mathrm{XRD}}^{\prime}\right] / w_{\mathrm{XRD}}^{\prime}
$$

where $w_{\mathrm{XRD}}^{\prime}$ parameterised the distance $(\approx 0.29 \mathrm{~nm})$ between neighbouring amino acids when the tissue is unstretched, derived from the width of a (predetermined) reflection and $w_{\mathrm{XRD}}$ is the distance between the neighbouring amino acids along the helical axis when the tissue is undergoing uniaxial extension (Fig. 5(A)). Of note, in the X-ray crystallographic study, ${ }^{106,107}$ the stress generated within a TC molecule is modeled by the ratio of the macroscopic force $\left(F_{\mathrm{WT}}\right)$ acting on an array of TC molecules connected by cross-links to the total cross-sectional area, $\alpha_{\mathrm{TC}} N$, of the TC molecules

$$
\sigma_{\mathrm{TC}}=F_{\mathrm{WT}} /\left[\alpha_{\mathrm{TC}} N\right]
$$

where $\alpha_{\mathrm{TC}}$ is the cross-sectional area of a TC molecule and $N$ the total number of TC molecules within the predetermined cross-section of the tissue which is identified, to order of magnitude, with

$$
N=A_{\text {TIS }} / S_{\text {LAT }}
$$

where $A_{\mathrm{TIS}}$ is the area of the tissue cross-section and $S_{\mathrm{LAT}}$ the area of a two dimensional unit cell for the transverse packing of TC molecules. These expressions provide a direct estimation of key mechanical parameters, namely $\sigma_{\mathrm{TC}}$ and the molecular axial deformation $w_{\mathrm{XRD}}-w_{\mathrm{XRD}}^{\prime}$. One immediate consequence is that this enables the Young modulus of TC molecule $\left(E_{\mathrm{TC}} \approx 2.9 \mathrm{GPa} \approx 3 \mathrm{GPa}\right)$ to be determined from the gradient of the stress-strain curve. More important, the results from MD simulation ${ }^{5,123,124}$ corroborate the findings of $E_{\mathrm{TC}}$ from XRD.

\section{Tropo-Collagen Molecular Interactions}

In this section, the Buehler approach for illuminating the mechanics of interactions among TC molecules is discussed. ${ }^{5,123,124}$ For simplicity we consider two adjacent TC molecules located within a collagen fibril (Fig. 6(A)). Two modes of deformation have been predicted using the bi-molecular mechanics approach; the first mode is known as the homogeneous shear while the second is known as the nucleation of slip pulses. The homogeneous shear mode explains how the TC molecules undergo sliding motion when a tensile load acts on the collagen fibril (Fig. 6(A)). Let $\tau_{\mathrm{TC}}$ represents the shear resistance between the two TC molecules. To order of magnitude, we can identify the $F$ (i.e., the axial force generated within the molecule) with the product of $\tau_{\mathrm{TC}}$ and $L_{\mathrm{CL}}$, i.e.,

$$
F=\tau_{\mathrm{TC}} L_{\mathrm{CL}}
$$

A

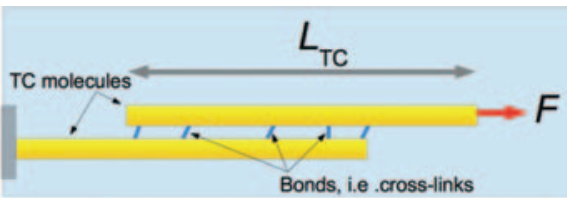

B
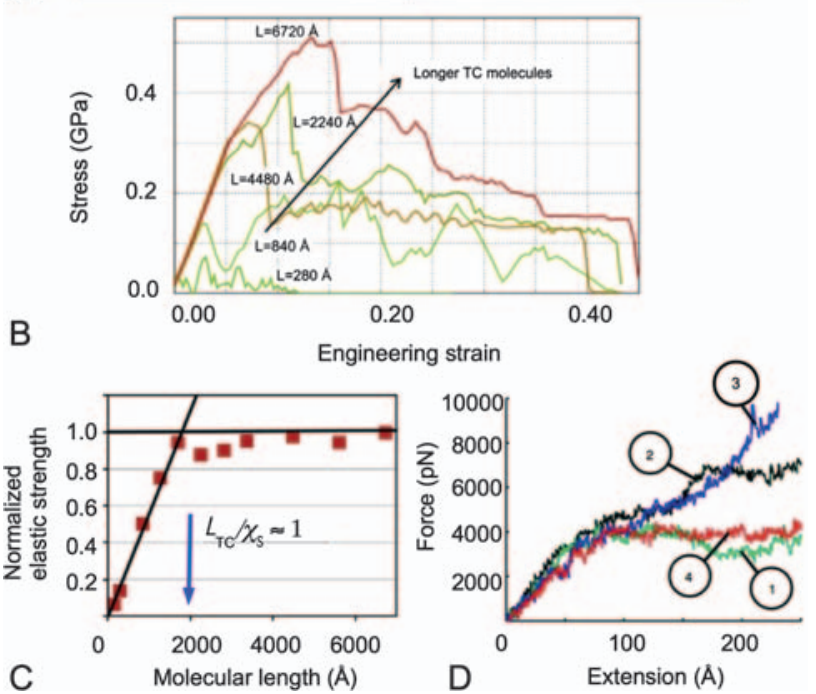

Figure 6. Predictions from the bi-molecular assembly of tropo-collagen (TC) molecules. (A) A model of the bi-molecular assembly of TC molecules. Symbols $F$ and $L_{\mathrm{TC}}$ represent the applied force and length of the molecule, respectively; blue lines represent covalent cross-links. (B) A sketch of the graph of stress versus engineering strain for different values of $L_{\mathrm{TC}}$ and (C) a sketch of the graph of normalized elastic strength versus, for a bi-molecular assembly of TC molecules. Symbol $\chi_{s}$ represents the first molecular length. Reprinted with permission from [5], M. J. Buehler, et al., Theoretical and computational hierarchical nanomechanics of protein materials: deformation and fracture. Progr. Mater. Sci. 53, 1101 (2008). (C) 2008, Elsevier. (D) A sketch of the graphs of force versus extension of a bi-molecular assembly of TC molecules corresponding to four different implementations of loading and cross-linking, labelled as case 1, 2, 3 and 4 . All results (B)-(D) were derived from molecular dynamics (MD) simulation. Reprinted with permission from [45], S. G. M. Uzel and M. J. Buehler, Molecular structure, mechanical behavior and failure mechanism of the C-terminal cross-link domain in type I collagen. J. Mech. Behav. Biomed. Mater. 4, 153 (2011). @ 2011, Elsevier.

or otherwise,

$$
F=\eta \tau_{\mathrm{TC}} L_{\mathrm{TC}}
$$

where $L_{\mathrm{TC}}$ is the length of a TC molecule, $L_{\mathrm{CT}}$ the contact length and $\eta$ parameterizes the fraction of contact length relative to the molecular length. The stress, $\sigma_{\mathrm{TC}}$, associated with $F$ is,

$$
\sigma_{\mathrm{TC}}=F / \alpha_{\mathrm{TC}}
$$

(recall $\alpha_{\mathrm{TC}}$ is the molecular cross-sectional area in Eq. (2)), and

$$
\eta=L_{\mathrm{CL}} / L_{\mathrm{TC}}
$$

Of note, $\eta \approx 3 / 4$ from $\mathrm{X}$-ray crystallographic studies. ${ }^{75}$ As the name suggests, the homogeneous shear case is 
valid when the shear deformation is uniformly distributed throughout the interface of the two TC molecules.

The second case, i.e., nucleation of slip pulses, explains how the rupture of intermolecular bonds, i.e., cross-links, leads to the propagation of slip pulses. According to Griffith's fracture energy argument of energy release rate (one dimensional model), at the onset of fracture, nucleation of slip pulses is controlled by the applied tensile stress, $\sigma_{\text {Griffith }}$, on the tissue,

$$
\sigma_{\text {Griffith }}=\sqrt{2 E_{\mathrm{TC}} \gamma_{\mathrm{TC}}}
$$

where $E_{\mathrm{TC}}$ is the Young modulus of an individual TC molecule (see Section Molecular force-displacement curve) and $\gamma_{\mathrm{TC}}$ parameterizes the energy required to nucleate a slip pulse.

When $\sigma_{\mathrm{TC}}<\sigma_{\text {Griffith }}$, the deformation of TC molecules is regulated by homogeneous shear (the homogeneous shear theory) between the TC molecules. When $\sigma_{\mathrm{TC}}>\sigma_{\text {Griffith}}$, nucleation of slip pulses occurs (i.e., the slip pulse theory). Putting all these together, we arrive at the first critical molecular length,

$$
\chi_{S}=\frac{\sqrt{2 E_{\mathrm{TC}} \gamma_{\mathrm{TC}}}}{\tau_{\mathrm{TC}} \eta} \alpha_{\mathrm{TC}}
$$

for predicting how the structure of the TC molecule influences the mechanics of TC molecular interaction. Simply put it, homogeneous shear dominates if $L_{\mathrm{TC}} \leq \chi_{S}$; nucleation of slip pulses dominates if $L_{\mathrm{TC}}>\chi_{S} \cdot{ }^{123}$ The strength of the fibril may be estimated to the order of magnitude with $\eta \tau_{\mathrm{TC}} \chi_{S}$ by replacing $L_{\mathrm{TC}}$ by $\chi_{S}$ in Eq. (5). ${ }^{123}$

An examination of Eq. (9) reveals that $\chi_{S}$ depends on the parameters related to the material properties and the interaction between molecules. In particular, $\gamma_{\mathrm{TC}}$ depends on the cross-link density and water content; the higher the density of cross-links (or equivalently low water content) the larger the value of $\gamma_{\mathrm{TC}}$. Thus the tensile strength (in terms of force) of the TC molecules, $F_{\max }$, is large if $\gamma_{\mathrm{TC}}$ is large.

Under an increasing $F$, eventually $F=F_{\max }$ and one of the two processes can occur to the TC molecules: homogeneous shear or nucleation of slip pulses. A second critical molecular length scale

$$
\chi_{R}=F_{\max } /\left[\tau_{\mathrm{TC}} \eta\right]
$$

governs the transition from molecular shear to brittle-like rupture of individual TC molecules. ${ }^{123}$ Thus, TC molecules will experience homogeneous shear if $L_{\mathrm{TC}} \leq \chi_{R}$; the individual TC molecule ruptures if $L_{\mathrm{TC}}>\chi_{R}$. Here, $\sigma_{\mathrm{Griffith}}$ depends on $L_{\mathrm{TC}}$, and is further constrained by $\chi_{S}$ and $\chi_{R}$. From the two length scale constraints, we may express these as a ratio, $\chi_{S} / \chi_{R}$, to address the conditions for nucleation of slip pulses or molecular rupture as follows: (1) when $\chi_{S} / \chi_{R} \leq 1$, slip pulses are generated; (2) when $\chi_{S} / \chi_{R}>1$, the individual TC molecule ruptures.
In addition, the bi-molecular model predicts a critical molecular length $L_{\min }$, defined by

$$
L_{\min }=\min \left(\chi_{R}, \chi_{S}\right)
$$

If $L_{\mathrm{TC}}<L_{\min }$, homogeneous shear dominates; if $L_{\mathrm{TC}}>$ $L_{\text {min }}$, either nucleation of slip pulses or molecular fracture dominates. Whether nucleation of slip pulses or molecular fracture dominates depends on which of the two, i.e., $\chi_{S}$ and $\chi_{R}$, is the smaller value (in accordance with Eq. (11)).

According to fracture mechanics, the work $\left(U_{\mathrm{TC}}\right)$ to separate the two TC molecules over $L_{\mathrm{CL}}$ is given by

$$
U_{\mathrm{TC}}=\int_{z=0}^{z=L_{\mathrm{CL}}} z \tau_{\mathrm{TC}} \mathrm{d} z
$$

This yields a simple expression for $U_{\mathrm{TC}}$, in terms of $L_{\mathrm{CL}}$ and $\tau_{\mathrm{TC}}$, as follows

$$
U_{\mathrm{TC}}=[1 / 2] L_{\mathrm{CL}} \tau_{\mathrm{TC}}^{2}
$$

This simple but important relationship suggests that the longer the TC molecule the more energy is required for shear deformation.

The predictions from the bi-molecular interaction model were developed further using MD simulation; the results of this investigation are plotted in Figures 6(B) and (C). Figure $6(\mathrm{C})$ reveals a mechanically saturated state for the normalized elastic strength of $F_{\max }$ at the $L_{\mathrm{TC}} \approx 200 \mathrm{~nm}$; this saturation reflects a change from homogeneous shear to propagation of slip pulses. The MD simulation reveals that $F_{\max } \approx 2.4 \times 10^{3} \mathrm{pN}$ and $\tau_{\mathrm{TC}} \approx 5.5 \mathrm{pN} / \mathrm{A}$; since this must be satisfied by $L_{\mathrm{TC}} / \chi_{S}=1$, we find that this corresponds to a critical molecular length $\chi_{S} \approx 200 \mathrm{~nm}$. From Eq. (10), $\chi_{R} \approx 436 \mathrm{~nm} .^{5}$ A simple analysis shows that $\chi_{S} / \chi_{R}=200 / 436 \approx 0.46<1$; in other words the nucleation of slip pulses could occur for the TC molecule.

The profile of the stress-strain curve from the bimolecular model (Fig. 6(B)) is sensitive to variation in $L_{\mathrm{TC}}$. Thus shorter $L_{\mathrm{TC}}$ yields smaller peak stress and vice versa. In other words, the longer the TC molecule the stronger is the interaction (higher stress uptake) between the TC molecules. Typically, $L_{\mathrm{TC}}$ is of the order of $300 \mathrm{~nm} .{ }^{5,123,124}$ One immediate consequence is that since $L_{\mathrm{TC}} \approx 300 \mathrm{~nm}>L_{\min }=\min (200 \mathrm{~nm}, 436 \mathrm{~nm})$, either nucleation of slip pluses (i.e., an intermolecular shear deformation) or molecular fracture dominates in Type I collagen molecule. More important, the Buehler model has provided the basis for understanding how TC molecules deform in tension when a SCT undergoes uniaxial extension in accordance with results from experiments (e.g., $\mathrm{XRD}^{106,107}$ and Raman spectroscopy ${ }^{19,121}$ ).

\section{Tropo-Collagen Mechanical Properties}

There are two important implications arising from the analysis of $E_{\mathrm{TC}}$ (see Section Molecular force-displacement curve and Eqs. (8) and (9)). First, it provides insight into 
the nature of the stiffness in TC molecules. From the perspective of X-ray crystallography, $E_{\mathrm{TC}}$ is attributed to the rate of change in the stress generated within the chain of amino acids with respect to the displacement (per unit length of the TC molecule) between neighbouring amino acids along the helical axis. ${ }^{106,107}$ Thus it is straightforward to determine $E_{\mathrm{TC}}$ from the stress-strain curve of a TC molecule. ${ }^{106,107}$ According to the principles of Brillouin scattering, the nature of $E_{\mathrm{TC}}$ is associated with the propagation of high frequency elastic waves along the TC molecule. ${ }^{125,126}$ The frequency of the elastic wave is of the order of $10^{10} \mathrm{~Hz}$ for collagen; in terms of wavelength, we find that this could range from $300 \mathrm{~nm}$ to $400 \mathrm{~nm}$ (which is consistent with the TC molecular length). Thus these elastic waves can interact with electromagnetic waves in the visible region. A simple relationship between $E_{\mathrm{TC}}$ and the elastic wave propagation velocity $(v)$ is given by

$$
E_{\mathrm{TC}}=\kappa v^{2}
$$

where $\kappa$ represents the density of the elastic (continuum) phase of the collagen. ${ }^{125,126}$ The MD simulation of the bimolecular interaction model predicts that $E_{\mathrm{TC}} \approx 7 \mathrm{GPa} .{ }^{123}$ To order of magnitude, this prediction corroborates the results derived from several studies, namely MD simulation study of a single TC molecule $\left(E_{\mathrm{TC}} \approx 6 \mathrm{GPa}\right.$, at a displacement of $1 \mathrm{~nm}$; see Fig. 5(D) inset), ${ }^{119}$ simultaneous mechanical testing and X-ray crystallography of whole tissue $\left(E_{\mathrm{TC}} \approx 2.9 \mathrm{GPa} \approx 3 \mathrm{GPa} \text {; see Fig. } 5(\mathrm{C})\right)^{106}$ and Brillouin scattering of whole tissue $\left(E_{\mathrm{TC}} \approx 5-9 \mathrm{GPa}\right){ }^{125,126}$

Second, $E_{\mathrm{TC}}$ is related to the persistence length, $L_{\mathrm{PL}}$. As explained elsewhere $L_{\mathrm{PL}}$ parameterizes (in terms of dimension) the tendency of the TC molecule to persist in a given direction, which has implication for how the TC molecule takes up load as it stretches. ${ }^{14,15}$ Accordingly, this leads to predictions concerning two deformation regimes. The first regime is known as the entropic elastic regime; it describes how the TC molecule undergoes displacement without stretching. A worm-like chain (WLC) argument presents the following relationship between $F$ and the extension experienced by the molecule, $u_{m}$

$$
\begin{aligned}
F\left(u_{m}\right)=k_{B} T /\left\{4 L_{\mathrm{PL}}\right\}[ & 1 /\left\{1-u_{m} / L_{\mathrm{con}}\right\}^{2} \\
+ & \left.4 u_{m} / L_{\mathrm{con}}-1\right]
\end{aligned}
$$

where $k_{B}$ is the Boltzmann constant, $T$ the absolute temperature and $L_{\text {con }}$ the contour length of the molecule when it is in a relaxed state (i.e., free from tension). ${ }^{16,72,128-130}$ Thus, $L_{\mathrm{PL}}$ underpins the relationship between $F$ and $u_{m}$. The second displacement regime is known as the intrinsic elastic regime. Here, the deformation of the TC molecule is modeled by ${ }^{72}$

$$
u_{m} / L_{\mathrm{con}}=1-\frac{1}{2} \sqrt{\frac{k_{B} T}{F\left(u_{m}\right) L_{\mathrm{PL}}}}+\frac{F\left(u_{m}\right)\left[2 r_{\mathrm{TC}}\right]^{2}}{16 k_{B} T L_{\mathrm{PL}}}
$$

By fitting the WLC equation to the force-extension measurements the value of $L_{\mathrm{PL}}$ was estimated as $\approx 14.5 \mathrm{~nm} .{ }^{14}$ To order of magnitude, the radius of the TC molecule, $r_{\mathrm{TC}}$, is identified with a range of values from $0.28 \mathrm{~nm}$ to $0.68 \mathrm{~nm}$ (depending on the model that is used to evaluate $\left.r_{\mathrm{TC}}\right) .{ }^{14}$ It follows that $E_{\mathrm{TC}}$ may be estimated from the following model ${ }^{14,131}$

$$
E_{\mathrm{TC}}=4 k_{B} T L_{\mathrm{PL}} /\left[\pi r_{\mathrm{TC}}^{4}\right]
$$

From Eq. (17), one finds that $E_{\mathrm{TC}}$ ranges from $350 \mathrm{MPa}$ (taking $r_{\mathrm{TC}} \approx 0.68 \mathrm{~nm}$ ) to $12.2 \mathrm{GPa}$ (taking $r_{\mathrm{TC}} \approx 0.28 \mathrm{~nm}$ ), assuming $L_{\mathrm{PL}}$ is equal to $14.5 \mathrm{~nm} .{ }^{14}$ We note that the prediction from this model (Eq. (17)) suggests that the $E_{\mathrm{TC}}$ of Type I collagen is sensitive to changes in $r_{\mathrm{TC}}$.

The measurement of the Young modulus for collagen is important from the point of view of understanding the pathology of patients that have been diagnosed with osteogenesis imperfect. It is said that certain mutations in type I collagen could lead to disruption of the interchain $\mathrm{H}$ bond, hence the unfolding of the collagen molecule. ${ }^{114,127}$ The unfolding of the TC molecule may lead to a mechanically unstable (inflated) structure with an overall increase in the radius of gyration of the molecule. To order of magnitude, we can identify the radius of gyration with $r_{\mathrm{TC}}$; in accordance with Eq. (17), we thus find that $E_{\mathrm{TC}}$ must decrease. A decrease in $E_{\mathrm{TC}}$ will affect the mechanical stability of the mutated molecule ${ }^{127}$ with consequential deleterious effects on fibrillogenesis, leading to the pathological changes.

\section{Role of Cross-Links}

The bi-molecular model (e.g., Fig. 6(A)) has been extended to investigate the modes of cross-link failure in collagen. ${ }^{45}$ Four different models corresponding to different loading and cross-link implementations were established to study the sensitivity of intermolecular crosslinks to the mechanical response of TC molecules; these are referred to as case 1,2, 3 and 4. Of note, (1) common to the four different models is that the end of the left TC molecule is held fixed; (2) cross-link was implemented at the C-terminal cross-link domain. Case 1 describes a model with no cross-link between the TC molecules and a force is applied to all three polypeptides of the rightmost TC molecule. Case 2 describes a model with cross-link between the TC molecules and a force acts on all three polypeptides of the rightmost TC molecule. Case 3 describes a model with cross-link between the TC molecules but the force acts only on the polypeptide chain that contains the cross-link. Lastly, case 4 describes a model with cross-link between the TC molecules but the force acts on the polypeptide chain that does not contain the cross-link. All models were solved to derive the forceelongation response curves (Fig. 6(D)).

In principle, the influence of cross-links on the mechanical response of the TC molecules is not appreciable at 
small elongations. ${ }^{5,45}$ However, beyond a critical axial displacement $(\approx 50 \AA)$, the force-elongation profile depends on the respective loading and cross-link implementations. ${ }^{45}$ With regards to case 1 versus case 2 and 3 , the results suggest that cross-link is responsible for enhancing the ability of the structure to take up high forces before failure. ${ }^{45}$ In case 2 and case 3, the intermediate plateau on the force-elongation curve is attributed to the unrolling of the telopeptide (i.e., at the end of an alpha helix chain, where cross-links are found). ${ }^{45}$ The unrolling of the end of the alpha helix chain has an immediate implication for facilitating the absorption of a proportion of the energy arising from the force to deform the tissue before stretching begins in the cross-linked domain. ${ }^{45}$ The force-elongation response curve of case 1 and 4 appear somewhat similar, albeit a small appreciable deviation at larger elongation. In the regime of large elongation, the response curve of case 2 features an initial plateau region (100-150 $)$ ), followed by an increase in the force up to $150 \AA$ before yielding occurs $(150-200 \AA)$; this is attributed to interstrand delamination. Additionally, case 4 also leads to interstrand delamination, which is observed to occur at a displacement of $\approx 150 \AA$.

\section{Microfibril Sliding Mechanics}

The supertwist and interdigitation features of the microfibril (see Section Microfibrils) are expected to contribute to the elastic behaviour of the collagen fibril during loading. From a design perspective, interdigitation with neighboring microfibrils enables collagen fibrils to absorb and transmit axial forces via the lysine-hydroxy-lysine crosslinks, by an analogy to a networked rope where each element of the array transmits force to the rest of the array via mechanical linkages. ${ }^{71}$ Interdigitation with neighbouring microfibrils could also facilitate the sliding of microfibrils with respect to each other, which is an important contributory factor to the viscoelasticity of a collagen fibril. ${ }^{73}$

Additionally, the characteristic right-handed twist of the microfibril provides structural stability to the microfibril system by absorbing torsional effects, shielding the reactive torque from disrupting the helical structure of the TC molecule. ${ }^{71,73}$ The mechanics of force transmission is explained by the turning moment on the super-twisted system which enables the torsional force to be transmitted through the intermolecular cross-links, e.g., homogeneous shear or slip-pulse, ${ }^{5,123,124}$ to neighboring TC molecules in neighboring microfibrils. ${ }^{63,71}$

Under normal physiological loading the shear action from sliding of TC molecules is limited by cross-links at the telopeptide region, i.e., the amino acid residue at the end of the alpha helix chain. ${ }^{73} \mathrm{~A}$ comparison of the contributions to sliding by the structural units, namely TC molecules and microfibrils, at the respective levels to the stress relaxation process of a collagen fibril reveals that the TC molecules slide more slowly (longer relaxation time) than the microfibrils. ${ }^{73}$ The differential relaxation times for TC molecules and microfibrils suggests that the process of reducing the stress taken up in collagen fibrils is regulated by microfibrils which takes place by relative sliding. ${ }^{73}$ As the microfibrils slide among themselves, since the sliding of TC molecules is slower than that of the microfibrils, this helps to regulate the mechanical stability of the microfibrils by slowly reducing the stress generated within a microfibril. ${ }^{73}$ These sliding behaviours at the respective levels of the microfibril and $\mathrm{TC}$ molecules are consistent with the argument that sliding will occur more readily between structural units that possess the weakest interaction. ${ }^{8}$ In principle, from molecular level upward, sliding occurs least readily between TC molecules owing to the relatively strong covalent crosslinks as compared to microfibrils (which possess fewer crosslinks between themselves as compared to TC molecules), collagen fibrils (in which DCN provides the mechanically linkage via weak bonds), collagen fibres (which interact with the interspersed cells and PG-rich matrix) and fascicles (in which interfascicular interactions occur at the endotendinous sheath). ${ }^{8}$

The discussion presented in these subsections has highlighted the results for the mechanics of low dimensional structural units, namely TC molecule and microfibril. In the case of the former, the discussion presented arguments for how the molecular stress uptake occurs when the molecule is stretched and when it interacts with neighbouring molecules. In the case of the latter, the argument focuses on the interaction of the microfibril with adjacent ones to help explain how this leads to torsional deformation and how collagen fibril uniaxial extension favours sliding between microfibril to sliding between TC molecules. In the next section, we discuss how stress uptake occurs in collagen fibril when it undergoes uniaxial extension.

\section{Collagen Fibril Mechanics}

\section{Fibril Stress-Strain Relationship}

A sketch of the graph of stress versus strain derived from stretching collagen fibrils using a MEMS is shown in Figure 7(A). ${ }^{132}$ The plot illustrates true stress versus Eulerian (Almansi) strain. Here, true stress is defined as the ratio of the force generated in the fibril over the instantaneous cross-sectional area of the fibril and Eulerian strain is expressed as

$$
\varepsilon_{\mathrm{EU}}=\left[\Delta L_{\mathrm{CF}} / L_{\mathrm{CF}}\right]\left\{1-\Delta L_{\mathrm{CF}} /\left[2 L_{\mathrm{CF}}\right]\right\}
$$

where $\Delta L_{\mathrm{CF}}$ represents the change in the half-length of the collagen fibril, $L_{\mathrm{CF}}$. From an engineering perspective, the magnitude of the true stress would be higher than that from the nominal stress parameter, owing to a shrinking cross-sectional area with increasing elongation. Of note, a shrinking cross-sectional area would implicate a continuous reduction in the disorder of the molecular packing. ${ }^{120,122}$ Nevertheless, the profile of the plot 


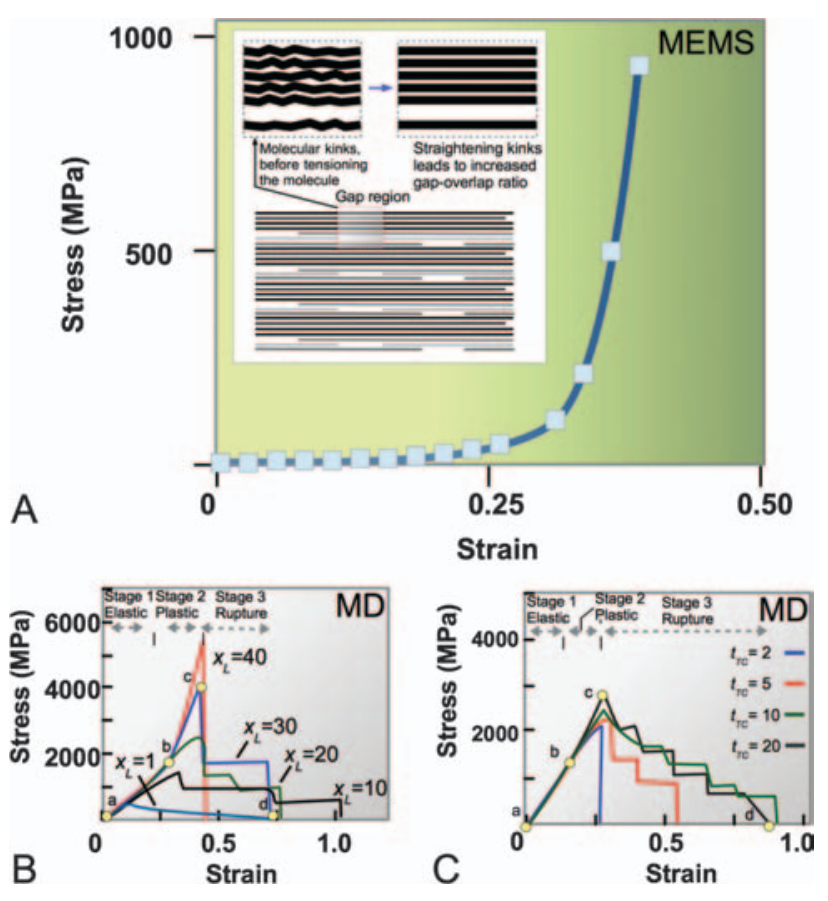

Figure 7. Stress-strain relationship of collagen fibrils. (A) A sketch of the graph of stress versus strain of collagen fibril derived from microelectromechanical system (MEMS). Reprinted with permission from [132], S. J. Eppell, et al., Nano measurements with micro-devices: Mechanical properties of hydrated collagen fibrils. J. R. Soc. Interface 3, 117 (2006). () 2006, Royal Society Publishing. (B) A sketch of the graph of stress versus strain for a network of tropo-collagen (TC) molecules to illustrate the effects of varying $X_{\mathrm{L}}$, a parameter for cross-link density. (C) A sketch of the graph of stress versus strain to illustrate the effects of varying $t_{\mathrm{TC}}$, a parameter for the lateral dimension of the TC molecular network. The results (B), (C) were derived from molecular dynamics (MD) simulation. Reprinted with permission from [135], Y. Tang, et al., Deformation micromechanisms of collagen fibrils under uniaxial tension. J. Roy. Soc. Interface 7, 839 (2010). ๑ 2010, Royal Society Publishing.

(Fig. 7(A)) is consistent with those obtained using the AFM system. ${ }^{46}$ The toe-to-heel region in these tissues is small and somewhat not appreciable. The toe-to-heel profile may be attributed to the relative axial displacement of the interdigitating microfibrils (see Section Microfibril sliding mechanics). At a lower level, it could also implicate the straightening of the helical structure of the TC molecule. ${ }^{133}$ Thereafter, as the load on the collagen fibril increases, a rapid increase in the stress in the collagen fibril is observed.

To understand how the underlying TC molecules contribute to the stress-strain relationship of the collagen fibril, a MD model comprising a three-dimensional network of staggered TC molecules, connected by crosslinks, was implemented to yield predictions of stress versus strain curves describing the mechanical response of the network of TC molecules. ${ }^{134-136}$ The model was evaluated by constraining the network in tension for different cross-link densities and network size. Here, crosslink density is parameterized by $X_{L}$, which is a dimensionless index for quantifying the extent of enhancing the adhesion forces between the beta-fold of the TC molecules. The network size was modeled to study the effects of varying the fibril lateral size on the mechanical response of a fibril. In this case the network size was parameterized by $t_{\mathrm{TC}} ; t_{\mathrm{TC}}$ quantifies the thickness of the network according to the number of stacked TC molecules.

A sketch of the graph of stress versus strain for a given longitudinal and lateral dimension of the molecular network to illustrate the effects of varying $X_{L}$ is shown in Figure 7(B). In addition, the effects of varying $t_{\mathrm{TC}}$ on the stress-strain relationship are shown in Figure $7(\mathrm{C})$ for a molecular network with a given $X_{L}(=20)$ and a longitudinal dimension that is similar to the network used in Figure 7(B). In general, the results suggest that cross-link density and fibril lateral size influence the strength (maximum stress) and toughness (strain energy density to rupture) of the collagen fibril. Although the maximum stress increases with increasing $X_{L}$ (as well as with increasing $t_{\mathrm{TC}}$ ), it appears that the maximum stress is more sensitive to the variations in $X_{L}$ than $t_{\mathrm{TC}}$. For instance, a four-fold increase in $X_{L}$ (from 10 to 40) produces a three-fold increase in the maximum stress (from 2000 to $6000 \mathrm{MPa}$ ). However, a ten-fold increase in $t_{T C}$ (from 2 to 20) leads only to 1.5 times increase in the maximum stress (from $2000 \mathrm{MPa}$ to $3000 \mathrm{MPa}$ ).

We present an argument to explain the underlying molecular mechanisms that direct the stress-strain profiles in Figures 7(B) and (C). This is illustrated using $X_{L}=20$ (Fig. 7(B)) and $t_{\mathrm{TC}}=20$ (Fig. 7(C)). In these instances, we note that the elastic stage (i.e., stage I) describes a somewhat linear elastic response from the origin (point a) to point b. The plastic stage (i.e., stage II) spans from point $\mathrm{b}$ to $\mathrm{c}$. The profile is characterized by an increase in the gradient. However, this increase in gradient is more appreciable in the case of $X_{L}=20$ (Fig. 7(B)) than in $t_{\mathrm{TC}}=20$ (Fig. 7(C)). Beyond the plastic stage, the stress decreases with further increase in strain; this effect is termed as strain softening. In most cases, strain softening depicts a gradual step-wise decrease in stress with increase in strain. Thus the molecular mechanism contributing to the deformation during the elastic stage (point a to b) is attributed to the molecular stretching arising from the uncoiling of the triple helical protein structures. ${ }^{100,131}$ Point b, which we refer to as the point of yielding, represents a critical transition from elastic state to plastic state. This transition defines the critical extension of a TC molecule such that the molecule would not be able to relax to its original state when the load acting on it is removed. Beyond point $b$, further increase in the applied load leads to the stretching of the covalent bonds in the collagen structure and this contributes to an increase in the gradient of the stress versus strain profile. ${ }^{134-136}$ Beyond the strain at maximum 


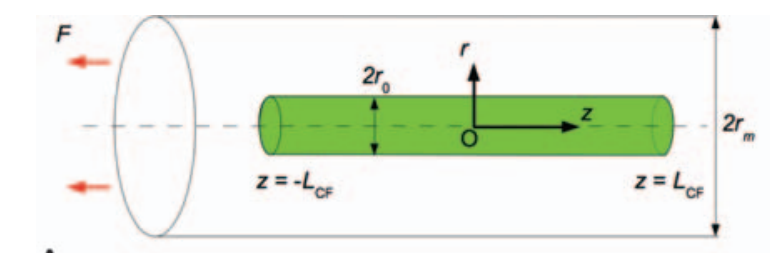

A

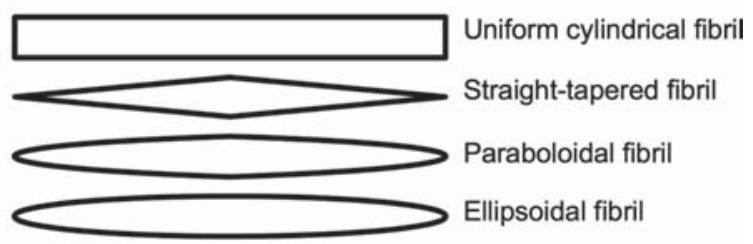

B

Figure 8. Schematics of collagen fibril. (A) A collagen fibril in $P G$-rich interfibrillar matrix. Symbols $z, r$ represent the axial and radial coordinates of the cylindrical polar coordinates system; $2 \mathrm{~L}_{\mathrm{CF}}, \mathbf{2} r_{0}$ : fibrillar length and diameter; $R$ is the interfibrillar distance; $F$ is the applied force on the tissue. (B) Collagen fibril profiles, namely uniform cylindrical shape, symmetrical straight-tapered, paraboloidal and ellipsoidal ends.

stress (i.e., point c), the strain softening effect is governed by a combination of rupture in the TC molecules (crosslinks disruption) and relative slip between TC molecules, leading to the failure of the network of TC molecules. Three possible dislocation-type slip systems, arising from the staggered nature of the network of TC molecules, have been identified. ${ }^{135}$

These stress-strain relationships of collagen fibrils provide the basis for our understanding of how collagen fibril takes up stress when uniaxially extended. In the following sections, we discuss how collagen fibril embedded in PGrich matrix (Fig. 8(A)) takes up stress at the different stages of the loading process. According to the order of occurrence, these stages are identified as elastic stress transfer, intermediate modes, plastic stress transfer and rupture. ${ }^{137-141}$ We highlight that the key parameters influencing the stress uptake at the respective stage are the fibril aspect ratio, $q$ (defined as the ratio of $L_{\mathrm{CF}}$ to the fibril radius at the fibril centre, $r_{0}$ ), the relative modulus of collagen to PG-rich interfibrillar matrix, $E_{f} / E_{P G}$, and the fibril shape. Consideration for the fibril shape assumes that uniform cylindrical fibrils, which forms the majority of the fibrils observed under an electron microscope, are found mainly in mature tissues while fibrils with tapered ends are found in developing tissues (Section Collagen fibrils). Although quantitative evidence has suggested that the ends resemble a paraboloid, ${ }^{41,43}$ the exact shape of the taper remain uncertain given that current techniques for isolating the fibrils could cause undue distortion to the taper, leading to the artifacts seen in the images from TEM. Nevertheless, we can attempt to understand how taper in fibrils enables the fibril for reinforcing ECM by studying a range of possible tapered shapes. Here, we focus on three different taper of symmetry at the fibril ends, namely the straight-tapered, paraboloidal and ellipsoidal ends (Fig. 8(B)). The results from these tapered ends would be compared with those from the uniform cylindrical fibrils to enable us to assess how taper influences the fibrillar stress uptake and hence, enabling insights into the sensitivity of stress uptake to varying fibril shapes to be gained.

\section{Elastic Stress Transfer}

The concept of elastic stress transfer ${ }^{142,143}$ has been applied to address how collagen fibrils reinforce ECM during initial loading, when the load acting on the SCT is small. ${ }^{140,141}$ Consider a collagen fibril embedded in and reinforcing PG-rich interfibrillar matrix (Fig. 8(A)) for the purpose of evaluating the elastic stress transfer stage. ${ }^{140,141}$ An external tensile load acts along the axis of the tendon to cause it to deform. Within the tendon, as the ECM deforms, the PG-rich interfibrillar matrix deforms in shear. Shear stress is generated on the surface of the collagen fibril. Since the PG-rich interfibrillar matrix is bonded to the collagen fibril, this causes the fibril to deform axially. The following assumptions are introduced. First, the PG-rich interfibrillar matrix and collagen fibril are both responding elastically to the external load; in other words, they are able to return to their original structural state when the load is removed. Second, the bonds (e.g., van Der Waals, hydrogen) are numerous and are distributed uniformly over the interface so that continuum mechanics can be used to analyze the problem. The axial elastic displacements in the fibril and in the PG-rich interfibrillar matrix will be different because of the difference in the elastic moduli of the two components. Shear strains are produced on all planes parallel to the axis of the fibrils in the direction of this axis. In order for the collagen fibrils to carry the bulk of the load acting on the tissue, the collagen fibril stiffness and strength would have to be larger than those of the PG-rich interfibrillar matrix. Consequently, it is expected that the deformation in the PG-rich interfibrillar matrix are larger than those in the collagen fibril. Adapting from the arguments based on engineering composites, ${ }^{142-144}$ if the tendon as a whole is subject to a strain of $\varepsilon$ in the direction of the fibril, the rate of change of the axial stress $\left(\sigma_{z}\right)$ along the fibril is proportional to the difference between the axial displacement of the fibril $\left(u_{f}\right)$ at any point within the fibril and the corresponding axial displacement of the PG-rich interfibrillar matrix at the same point if the fibril were not presence, $\left(u_{\mathrm{PG}}\right)$, i.e.,

$$
\mathrm{d} \sigma_{z}(Z) / \mathrm{d} Z=H\left[u_{f}-u_{\mathrm{PG}}\right]
$$

where $H$ is constant, and $Z\left(=z / L_{\mathrm{CF}}\right)$ is the normalized axial coordinate $(z)$ which parameterizes the axial distance of the fibril starting from the fibril centre $(z=0)$ and ending at the fibril end, i.e., $z=L_{\mathrm{CF}}$. Solving Eq. (19) for uniform cylindrical fibrils, we find that $\sigma_{z}$ and interfacial 
shear $(\tau)$ stress generated at the collagen fibrillar surface are given by

$$
\begin{gathered}
\sigma_{z}(Z)=E_{f} \varepsilon[1+\cosh (\beta\{1-Z\}) / \cosh (\beta)] \\
\tau(Z)=E_{f} \varepsilon \sqrt{\frac{G_{\mathrm{PG}}}{E_{f} 2 \ln \left(r_{\mathrm{PG}} / r_{0}\right)}} \frac{\sinh (\beta\{1-Z\})}{\cosh (\beta)}
\end{gathered}
$$

respectively, ${ }^{138,140,141,145}$ where,

$$
\beta=\sqrt{\frac{L_{\mathrm{CF}}^{2} H}{E_{f} A_{f}}}
$$

or, in terms of the collagen tensile stiffness, $E_{f}$, and shear modulus of the PG-rich interfibrillar matrix, $G_{\mathrm{PG}}$,

$$
\beta=\sqrt{\frac{G_{\mathrm{PG}} 2 \pi L_{\mathrm{CF}}^{2}}{E_{f} A_{f} \ln \left(r_{\mathrm{PG}} / r_{0}\right)}}
$$

Let $E_{\mathrm{PG}}$ be the tensile stiffness of the PG-rich interfibrillar matrix. Of note, $E_{f} / E_{\mathrm{PG}}$ may be expressed in terms of $G_{\mathrm{PG}} / E_{f}$, giving

$$
E_{f} / E_{\mathrm{PG}}=\left\{E_{f} / G_{\mathrm{PG}}\right\} /\left\{2\left[1+v_{\mathrm{PG}}\right]\right\}
$$

where $v_{\mathrm{PG}}$ is the Poisson ratio of the PG-rich interfibrillar matrix.
A finite element (FE) model of a uniform cylindrical collagen fibril similar to that shown in Figure 8(A), undergoing elastic stress transfer, was used to solve for the $\sigma_{z}$ and $\tau$ in the fibril to study the sensitivity of these stresses to variation in the fibril aspect ratio, $q\left(=L_{\mathrm{CF}} / r_{0}\right)$ and $E_{f} / E_{\mathrm{PG}} \cdot{ }^{138-141,146}$ A sketch of the normalized axial stress, $\sigma_{z} / \sigma_{c}$, versus $Z$ derived from the FE analysis is shown in Figure 9(A) for the case of $q=1000$ and $E_{f} / E_{\mathrm{PG}}=$ $10^{4} .^{138,146}$ (Here, $\sigma_{c}$ represents the applied stress on the tissue). A sketch of the corresponding normalized shear stress, $\tau / \sigma_{c}$, versus $Z$, is shown in Figure $9(\mathrm{~B})$. For the purpose of illustration, these values for $q$ and $E_{f} / E_{\mathrm{PG}}$ have been chosen to lie in between the extremes of $q$ and $E_{f} / E_{\mathrm{PG}}$ found in SCTs. ${ }^{138-141}$ These profiles of axial and shear stress distributions corroborate the predictions from Eqs. (20) and (21). Thus, the interfacial shear stress, $\tau / \sigma_{c}$, is a minimum at the fibril centre; the magnitude of the $\tau / \sigma_{c}$ increases non-linearly until it reaches a maximum at the fibril end. Concomitantly, the fibril axial stress, $\sigma_{z} / \sigma_{c}$, is greatest at the fibril centre and falls linearly to zero at the fibril end (Fig. 9(A)).

The stresses in a fibril with tapered ends, corresponding to symmetrical straight-tapered, paraboloidal and ellipsoidal ends, have also been investigated by FE analysis because no feasible analytical solutions have been

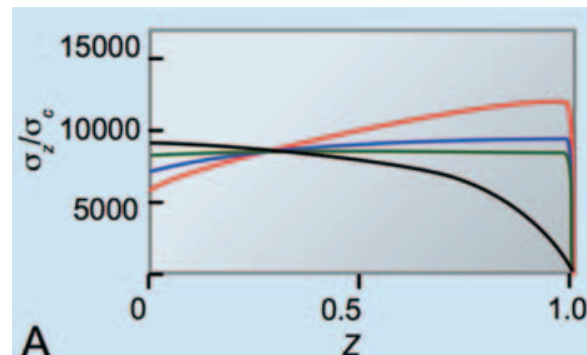

A

Z

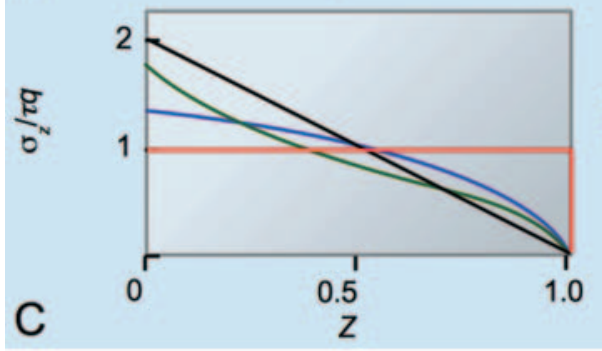

- Straight-taper — Paraboloidal

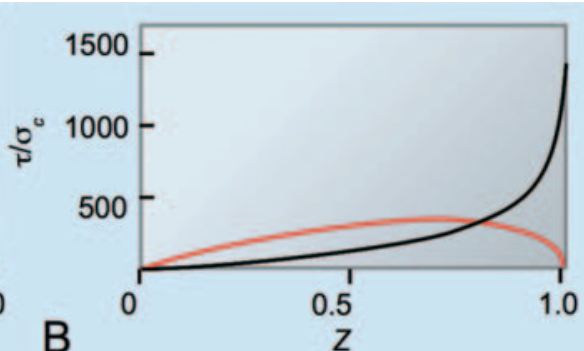

B

Z

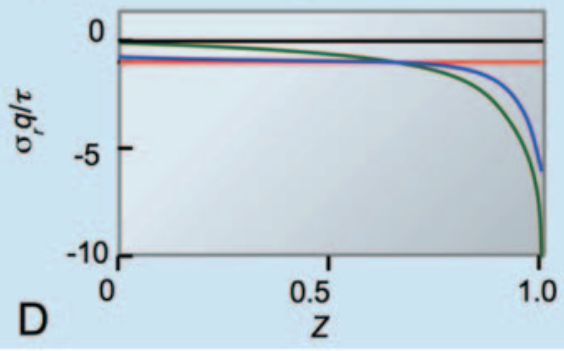

Ellipsoidal - Uniform cylindrical

Figure 9. Stresses in a collagen fibril during elastic (A), (B) and plastic (C), (D) stress transfer. (A) A sketch of the graph of normalized axial stress, $\sigma_{z} / \sigma_{c}$, versus fractional distance, $Z$, along the fibril. Reprinted with permission from [138], K. L. Goh, et al., Finite-element analysis of the effect of material properties and fibre shape on stresses in an elastic fibre embedded in an elastic matrix in a fibre-composite material. Proc. Roy. Soc. Lond. Math. Phys. Sci. 460, 2339 (2004). ( 2004, Royal Society Publishing. (B) A sketch of the graph of normalized interfacial shear stress, $\tau / \sigma_{c}$, versus $Z$. The shear stress distributions from the paraboloidal and ellipsoidal fibrils overlap the stress distribution of the uniform cylindrical fibril. Adapted with permission from [140], K. L. Goh, et al., Influence of fibril taper on the function of collagen to reinforce extracellular matrix. Proc. Roy. Soc. Lond. Biol. Sci. 272, 1979 (2005), ( $)$ 2005. (C) A sketch of the graph of normalized axial stress, $\sigma_{z} / \tau_{q}$, versus $Z$. (D) A sketch of the graph of normalized radial stress, $\sigma_{r} q / \tau$, versus $Z$. Symbol $q, \tau$ and $Z$ represent the fibril aspect ratio, the interfacial shear stress on the SCT and the fractional distance along the fibril axis from the centre $(Z=0)$ to the end $(Z=1)$ of the fibril, respectively. Reprinted with permission from [137], K. L. Goh, et al., Effect of fibre shape on the stresses within fibres in fibre-reinforced composite materials. Proc. Roy. Soc. Lond. Math. Phys. Sci. 455, 3351 (1999). @ 1999, Royal Society Publishing. 
found by solving the Eq. (19) for fibril with tapered ends. ${ }^{140,141,146}$ A sketch of the graphs of $\sigma_{z} / \sigma_{c}$ versus $Z$ for the respective fibril shapes is illustrated in Figure 9(A); the corresponding graphs of $\tau / \sigma_{c}$ versus $Z$ is illustrated in Figure $9(B)$. Consider the axial stress distributions in Figure 9(A). Thus the effect of a taper, in comparison with a uniform cross-section, is to reduce the axial stress at the fibril centre. ${ }^{138-141}$ As observed in all the fibrils with tapered ends, the magnitude of $\sigma_{z} / \sigma_{c}$ (starting from the fibril centre) increases as $Z$ increases; at the fibril end, $\sigma_{z} / \sigma_{c}$ falls rapidly to zero because no force transmission occurs across the fibril ends. Among the three different tapers, it was found that the $\sigma_{z} / \sigma_{c}$ distribution of the ellipsoidal fibril is the most uniform. ${ }^{138-141}$

Consider the shear stress distribution in Figure 9(B). The profile of the graph reveals a characteristic minimum value (i.e., $\left.\tau / \sigma_{c}=0\right)$ at the fibril centre $(Z=0)$ for all shapes. ${ }^{146}$ Thereafter, except for the fibril with straighttapered ends, the magnitude of $\tau / \sigma_{c}$ increases non-linearly to a maximum at the fibre end $(Z=1) .{ }^{146}$ In fact, the graphs of the $\tau / \sigma_{c}$ distribution for fibrils with ellipsoidal ends, paraboloidal ends and the uniform cylindrical fibril overlap for the most part of the fibril surface. ${ }^{146}$ For the fibril with straight-tapered ends, the magnitude of $\tau / \sigma_{c}$ peaks at a distance between the fibril end and the fibril centre. ${ }^{146}$ Altogether, the analysis of the $\tau / \sigma_{c}$ distributions of the respective fibril shape is important because it lends support to our understanding of how the interface could be disrupted, e.g., debonding and delamination, when the interface shear stress exceeds the shear strength.

The FE analysis also reveals the sensitivity of the $\sigma_{z} / \sigma_{c}$ distribution to variation in $q$ and $E_{f} / E_{\mathrm{PG}} \cdot{ }^{138-141}$ In particular, we highlight that (i) the profile of the $\sigma_{z} / \sigma_{c}$ distribution is sensitive to variation in $q$ and (ii) the magnitude of the $\sigma_{z} / \sigma_{c}$ is sensitive to variation in $E_{f} / E_{\mathrm{PG}}$. In other words, increasing $E_{f} / E_{\mathrm{PG}}$ for a fixed value of $q$ has an appreciable effect on the magnitude of the axial stress but little effect on the profile of the axial stress distribution along the fibril axis. ${ }^{138-141}$ Large values of $E_{f} / E_{\mathrm{PG}}$ result in greater stresses (for a fixed value of $q$ ) ${ }^{138-141}$ On the other hand, increasing $q$ (for a fixed value of $E_{f} / E_{\mathrm{PG}}$ ) has an appreciable effect on the profile of the axial stress distribution. Large values of $q$ result in more uniform distribution of axial stress along the fibril axis. ${ }^{138-141}$

One advantage of tapered ends over uniform cylindrical ends is that this enables the fibril to make more effective use of the collagen synthesized by the cells in the tissue. As pointed out in previous paragraph, for a tapered fibril, the $\sigma_{z}$ distribution is more uniform along its length. This greater uniformity enables greater use of the full length of the fibril in reinforcing the ECM. For a given $L_{\mathrm{CF}}$ and $r_{0}$, the volumes of the respective fibril shapes, namely uniform cylinder, straight-taper, paraboloid and ellipsoid are as follows: $\pi r_{0}^{2} L_{\mathrm{CF}},[1 / 3] \pi r_{0}^{2} L_{\mathrm{CF}},[1 / 2] \pi r_{0}^{2} L_{\mathrm{CF}}$ and $[2 / 3] \pi r_{0}^{2} L_{\mathrm{CF}}$. Thus, the volume of a uniform cylindrical fibril is twice that of a paraboloidal fibril. ${ }^{147}$ In other words, half as much collagen is required to make a paraboloidal fibril as a uniform cylindrical fibril of the same length. Interestingly, since all arguments point to fibrils with tapered ends as more effective than uniform cylindrical fibrils for reinforcing ECM, from an optimal design perspective, it follows that ECM of developing to old individuals should ideally be predominated by fibrils of the former shape.

\section{Intermediate Modes of Failure}

In the transition from elastic stress transfer (see Section Elastic stress transfer) to plastic stress transfer (see Section Plastic stress transfer), three possible intermediate modes of failure, termed mode $\alpha, \beta$ and $\gamma$, may occur. These intermediate modes of failure are analogous to those found in engineering composites. ${ }^{139}$ While these failure modes are well-known in engineering composites, it is still not well understood in SCTs. In the following paragraphs, we outline the possible scenarios that could happen at the respective failure modes.

Mode $\alpha$ is said to occur when the deforming PG-rich interfibrillar matrix yields and turns plastic but only in the region adjacent to the fibril surface where bonding exists at the interface. In the case of uniform cylindrical fibrils, stress concentrations in the PG interfibrillar matrix around the fibrillar end may lead to matrix yielding. ${ }^{148}$ Fibrils with straight-tapered ends may be less susceptible to mode $\alpha$ because of the lower stress concentrations in the PG-rich interfibrillar matrix in the vicinity of the fibrillar ends. ${ }^{149}$ Nevertheless, should mode $\alpha$ occur, the PGrich interfibrillar matrix responds like an elastic-plastic material. ${ }^{148,150,151}$ In other words, initially, the stress in the PG-rich interfibrillar matrix would increase with increasing strain (elastic) until yielding occurs, and this is followed by little or no increase in stress with increasing strain (plastic).

Mode $\beta$ is characterized by the initiation of interfacial debonding. Debonding starts at the fibril end and propagates along the interface. In addition, as the deforming PG-rich interfibrillar matrix slides over the fibril surface, this enables frictional stress transfer to take place. ${ }^{150}$ The rate of debonding increases with $E_{f} / E_{\mathrm{PG}}$, which parameterizes the elastic mismatch between the fibril and PG-rich interfibrillar matrix. ${ }^{152}$ Mode $\beta$ takes place when the $\tau$ (which arises from the stretching of bonds at the interface between the fibril and PG-rich interfibrillar matrix) increases until it reaches the yield point (parameterized by the yield stress in shear, $\tau_{y}$ ) as the load acting on the SCT increases. From continuum mechanics, it follows that $\tau_{y}$ is regulated by the frictional stress, $\mu N$ ( $\mu$, coefficient of friction; $N$, normal force acting on the interface) and a cohesive sliding resistance. As the load acting on the SCT increases further, eventually $\tau$ exceeds $\tau_{y}$ and delamination of the two surfaces occurs. Consequently, the PG-rich interfibrillar matrix can now slide easily over the fibril 
surface. During the process of sliding, the value of $\tau$ is constant throughout the interface, i.e., ${ }^{139}$

$$
\tau(Z)=\left\{\begin{array}{cc}
\tau_{0} & 0 \leq Z \leq 1 \\
-\tau_{0} & -1 \leq Z<0 \\
0 & \text { elsewhere }
\end{array}\right.
$$

Of note, at the molecular level, a constant value for the magnitude of $\tau$, along the interface implies a constant number of interactions per unit area between interfibrillar matrix and the fibril surface. ${ }^{140,141}$ In order for shear sliding to occur at the interface, this would involve overcoming the intermolecular forces at the interface. ${ }^{140,141}$ One may assume that the number of interactions per unit area is constant if there is no appreciable variation in the composition of the PG-rich interfibrillar matrix along the fibril surface. ${ }^{140,141}$ Nevertheless, according to continuum mechanics $\tau$ is regulated by the kinetic frictional stress under these circumstances. Additionally, $\tau$ would be smaller than $\tau_{y}$ at this stage.

Mode $\gamma$ is said to occur when the initiation of rupture at the debonded fibril end propagates into the PGrich interfibrillar matrix (see schematic in Fig. 11(A)) instead of along the interface between the fibril and the matrix. ${ }^{153}$ According to fracture mechanics, two different modes of rupture could result from mode $\gamma$ : cracking, i.e., parting of two surfaces (conventionally termed as mode I), and shear failure (conventionally termed as mode II). If the former occurs, this would reduce the effectiveness of stress transfer between the PG-rich interfibrillar matrix and fibril because stress transfer will not occur across the crack planes; if the latter occurs, this would enabled stress to be transferred via friction at the crack surfaces. ${ }^{153}$

A sketch of a graph of $\sigma_{z}$ versus $Z$ is shown in Figure 10(A). The corresponding graph of $\tau$ versus $Z$ is shown in Figure 10(B). These stress distributions represent a 'snap-shot' of the state of progression of deformation under an increasing applied load whereby one or more intermediate modes are occurring at the same time. In this case, the stress distributions feature three distinct zones on the fibrils. Starting with the zone (i.e., zone 1) near the fibril end $(Z=1)$, the stress profile is the result of mode $\beta$. We recall that the initiation of PG-rich interfibrillar matrix failure (mode $\alpha$ ) occurs at the fibril end. Thus, at the beginning of failure, it must be emphasized that only two zones are observed and they correspond to mode $\alpha$ and elastic stress transfer. However, when the high shear stress at the interface near the fibril end eventually leads to interfacial debonding (and so mode $\beta$ ensues), then mode $\alpha$ recedes from this zone and migrates to the adjacent zone (i.e., zone 2). Thus, regions around the fibril centre $(Z=0)$ will be free from high $\tau$. Accordingly elastic stress transfer dominates as long as the PG-rich interfibrillar matrix remains in the elastic state. (Of note, the stress distribution associated with fibril pullout (see Section Fibril pullout), may also be attributed to the mixed mode feature discussed here.) It must be emphasized that the $\sigma_{z}$ profile associated with mode $\beta$ is linear (Fig. 10(A)) because it is contributed by frictional shear stress at the interface, which arises from the sliding of the deforming PG-rich interfibrillar matrix over the fibril surface. Note also that mode $\alpha$ is associated with a linear profile of $\sigma_{z}$ versus $Z$; the corresponding $\tau$ is constant. These stress distributions are the simplest representations that we could use to model mode $\alpha$. Nevertheless, the constant shear in mode $\alpha$ is a reminder that the PG-rich interfibrillar matrix is behaving as a perfectly plastic material, i.e., the matrix yields under constant stress.

Finally, as pointed out earlier, mode $\gamma$ occurs when the plastically deforming PG-rich interfibrillar matrix (near the debonded fibril end) initiates a crack which originates from the debonded fibril end and propagates into the matrix. The crack morphology would look like a frustum, making an angle of less than $90^{\circ}$ with respect to the fibril axis. How mode $\gamma$ influences the stress distribution along the fibril axis is not well understood and is a subject for further investigation.

\section{Plastic Stress Transfer}

At higher loads, eventually the deforming PG-rich interfibrillar matrix around the fibril becomes plastic. Bonds at the fibril-matrix interface are disrupted and the PG-rich interfibrillar matrix 'shear-slides' over the surface of the fibril. ${ }^{137,140,154}$ Overall, plastic stress transfer is similar to mode $\beta$ but the key difference is that the bulk of the PG-rich interfibrillar matrix surrounding the delaminated interface is in a plastic state. According to continuum mechanics, normal forces are generated on the surfaces of the PG-rich interfibrillar matrix and the fibril; the corresponding frictional forces acts to resist the sliding action. Let $r$ represents the fibril radius; in general this is a function of $Z$. Of note, for uniform cylindrical fibrils, $r$ is constant $\left(=r_{0}\right)$. We further note that the product of $\sigma_{z}$ and $r^{2}$ is related to the axial reaction acting along the fibril. It follows that the rate of change of $\sigma_{z} r^{2}$ with respect to $Z$ is proportional to $\tau$ and $q,{ }^{137}$ i.e.,

$$
d\left[\sigma_{z} r^{2}\right] / \mathrm{d} Z=2 \tau q
$$

The corresponding radial stress, $\sigma_{r}$, at any point on the surface of the fibril, is proportional to the rate of change of $r$ at that point with respect to $Z,{ }^{137}$ i.e.,

$$
\sigma_{r}(Z)=-\tau \mathrm{d} r / \mathrm{d} Z
$$

Equation (26) is a generalization of the form for a uniform cylinder. ${ }^{143,154}$ To solve the differential equations, we note that flow of the PG-rich interfibrillar matrix along the fibril induces an interfacial (constant) shear stress given by Eq. (25). ${ }^{137,140,154}$ Solving the differential equation, 
Table I. Solutions to the plastic stress transfer problem.*

\begin{tabular}{lccc}
\hline Fibril shape & $r$ & $\sigma_{z}$ & $\sigma_{r}$ \\
\hline Uniform cylinder & $L_{\mathrm{CF}} / q$ & $2 \tau q\{1-Z\}$ & 0 \\
Straight-taper & {$\left[L_{\mathrm{CF}} / q\right]\{1-Z\}$} & $\tau q$ & $-\tau / q$ \\
Paraboloidal & {$\left[L_{\mathrm{CF}} / q\right] \sqrt{1-Z}$} & {$[4 / 3] \tau q \sqrt{1-Z}$} & $-(1 / 2)[\tau / q]\{1 / \sqrt{1-Z}\}$ \\
Ellipsoidal & {$\left[L_{\mathrm{CF}} / q\right] \sqrt{1-Z^{2}}$} & $\tau q\left\{\frac{\pi / 2-\sin ^{-1} Z}{1-Z^{2}}-\frac{Z}{\sqrt{1-Z^{2}}}\right\}$ & $-\frac{\tau}{q}\left\{\frac{Z}{\sqrt{1-Z^{2}}}\right\}$ \\
\hline
\end{tabular}

Notes: *Here, $\tau$ represents the interfacial shear stress during plastic stress transfer, $\sigma_{z}$ and $\sigma_{r}$ parameterise the axial and radial (surface) stresses in a collagen fibril as a function of the fractional axial distance, $Z\left(=z / L_{\mathrm{CF}}\right), L_{\mathrm{CF}}$ one-half the fibril length, $q$ the fibril aspect ratio $\left(L_{\mathrm{CF}} / r_{0}\right), r_{0}$ the radius at the fibril centre.

one arrives at expressions for the axial and radial stresses shown in Table I. ${ }^{137}$ These expressions were used to generate the graphs of $\sigma_{z}$ versus $Z$ for the respective fibril shapes as shown in Figure 9(C); the corresponding graphs of $\sigma_{r}$ versus $Z$ are shown in Figure 9(D). Consider the $\sigma_{z}$ distributions in Figure 9(C). For a uniform cylindrical fibril, the distribution of $\sigma_{z}$ yields a maximum $\sigma_{z}$ at $Z=0$ but this linearly decreases to 0 at $Z=1$. For a fibril with paraboloidal ends the stress rises more rapidly near the ends but less steeply near the centre. The overall result is that fibrils with tapered ends generate more even stress distribution along the fibril length and that the stress at the centre of the fibril is smaller than that in the uniform cylindrical fibril. Indeed the stress at the centre of a uniform cylindrical fibril is 1.5 times that at the centre of a fibril with straight-tapered ends.

Consider the $\sigma_{r}$ distributions in Figure 9(D). For a fibril with straight-tapered ends, $\sigma_{r}$ decreases linearly with $Z$, leading to a uniform compressive stress distribution. However, at the end of the fibre $\sigma_{r}=0$, so that there is a discontinuity in the distribution. For the paraboloidal and ellipsoidal fibrils, the compressive stress distribution is non-uniform and tends to infinity as $\sigma_{r}$ tends to zero. However, for the uniform cylindrical fibril, $\sigma_{r}$ is zero throughout the fibril surface. The prediction of $\sigma_{r}$ has an important and immediate consequence concerning the compressive stress acting on the TC molecules during accretion. It was pointed out that lateral accretion of mass occurs throughout the fibril (i.e., from the central shaft to the tip), resulting in an overall constant tip shape (see Section Collagen fibrils). We speculate that the constant tip shape of a growing fibril could be assisted by a constant (non-zero) compressive stress, ensuring that any TC molecules generated during accretion are drawn sufficiently near to the fibril surface in order for bonding to take place. On the other hand, how the non-uniform compressive stress in fibrils with paraboloidal and ellipsoidal ends, as well as the high compressive stresses at the tips, could assist in regulating tip growth is not well understood.

\section{Fibril Pullout}

Fibril pullout and fibril rupture can occur around the ruptured site of the PG-rich interfibrillar matrix. A schematic illustrating these failure patterns is shown in Figure 11(B). The fracture morphology has been a subject of intensive study. ${ }^{108,109,155-157}$ Fibril pullout may occur when fibrils are drawn out from the faces of a crack in the PG-rich interfibrillar matrix. One possible route to fibril pullout could come directly from the intermediate modes, namely a combination of modes $\beta$ and $\gamma$ (see Section Intermediate modes of failure), by-passing the plastic stress transfer stage (see Section Plastic stress transfer). However, in the absence of mode $\beta$, the crack in the PG-rich interfibrillar matrix arising from mode $\gamma$ may propagate to neighbouring fibrils. Given that the fibrils are now responsible for bridging the ruptured sites in the PG-rich interfibrillar matrix, this could lead to fibril pullout as the load on the SCT increases. Ultimately how a fibril bridges a PG-rich matrix ruptured site depends on the nature of the interface, ${ }^{154,158}$ the fibril strength, $\sigma_{f},{ }^{158}$ and $E_{f} / E_{\mathrm{PG}} \cdot{ }^{139}$ If bonding between the fibril and PG-rich matrix is sustained at the interface, then the fibril bridging the crack will slide out when the interfacial shear stress equals $\tau_{y}$ (i.e., when debonding occurs). Under these circumstances, how the fibril maintains the bridge across the ruptured site depends on the sliding friction. ${ }^{154,159}$ When fibril pullout occurs the rupture in the PG-rich interfibrillar matrix widens but this may be temporarily halted by neighbouring fibrils. In other words, the propagation of the rupture may be 'deflected' by these fibrils, and consequently, the rupture is constrained to propagate along the interface of these fibrils (mode $\beta$ ). ${ }^{143}$ However, if fibril pullout did not take place, then fibril fracture may occur when attempts to bridge the ruptured site leads to high stress uptake beyond $\sigma_{f}$. We shall discuss fibril fracture in the next section.

A sketch of the graph of $\sigma_{z}$ versus $Z$ for a collagen fibril undergoing pullout is shown in Figure $10(\mathrm{C})$. The corresponding graph of $\tau$ versus $Z$ is shown in Figure 10(D). These graphs are compiled from results from Raman spectroscopy, FE analysis and analytical models. ${ }^{159-161}$ The graphs describe the stress distribution within the fibril, starting from the embedded fibril end, $Z=1$, and terminating at the point of exit at the matrix crack plane, $Z=0$. The $\sigma_{z}$ distribution may be divided into three zones. The linear dependence of $\sigma_{z}$ with $Z$ in zones 2 and 3 is 


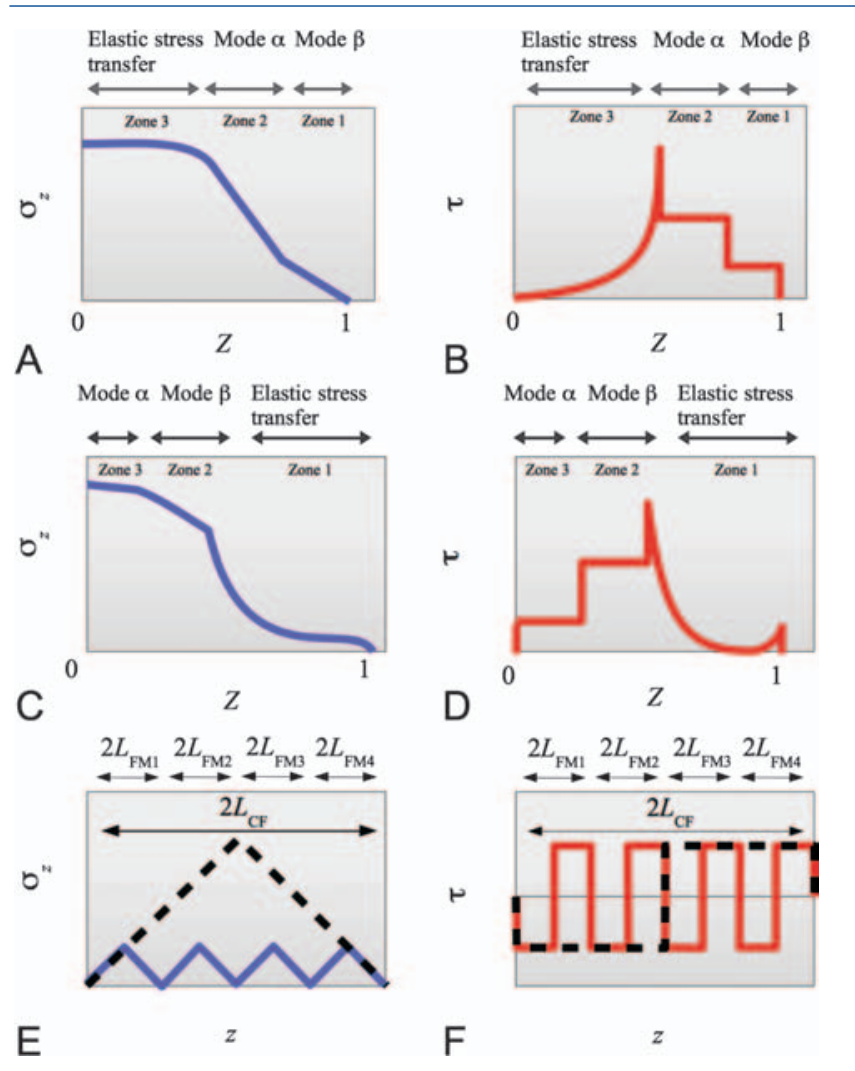

Figure 10. Stresses in a collagen fibril during the intermediate modes of stress transfer $(A),(B)$ and during fibril pullout (C), (D) and rupture (E)-(F). (A) A sketch of the graph of fibril axial stress, $\sigma_{z}$, and (B) the corresponding interfacial shear stress, $\tau$, versus the fractional axial distance, $Z$. A combination of elastic stress transfer, mode $\alpha$ and $\beta$ lead to the stress up-take in the fibril. (C) A sketch of a graph of $\sigma_{z}$ and (D) the corresponding graph of $\tau$ versus $Z$, during fibril pullout. Similarly, a combination of elastic stress transfer, mode $\alpha$ and $\beta$ lead to the stress up-take in the fibril. (E) A sketch of the graphs of $\sigma_{z}$ and $(F)$ the corresponding graphs of $\tau$ versus axial distance, $z$, of an intact fibril (dashed line) and after it ruptured into four fragments (solid line) lined end-to-end. Symbol $Z$ represents the fractional distance along the fibril axis from the centre $(Z=0)$ to the end $(Z=1)$ of the fibril. In the case of fibril rupture, the axes of the intact fibril and fibril fragments define the $z$-axis of the cylindrical polar coordinate system; $z$ represents the coordinate of the $z$-axis. Symbols $2 L_{1}$, $2 L_{2}, 2 L_{3}$ and $2 L_{4}$ represent the lengths of the respective fragments. Reprinted with permission from [139], K. L. Goh, et al., Review: Finite element analysis of stress transfer in short-fibre composite materials. Compos. Sci. Technol. 64, 1091 (2004). (c) 2004, Elsevier.

attributed to sliding action when debonding at the interface has occurred (i.e., mode $\beta$ ); the stress distribution in zone 1 arises from elastic stress transfer. ${ }^{159}$ Interestingly, the different gradients at zones 2 and 3 correspond to different levels of frictional sliding. The magnitude of $\sigma_{z}$ at the point of transition from zone 2 to 1 then rapidly decreases (non-linearly) with increasing $Z$ but instead of decreasing to zero, the stress plateaus out for a part of the fibril length before decreasing to zero at the fibril end. For the corresponding $\tau$ distribution in Figure 10(D), a peak value is located at the point of transition from zone 2 to 1 . In principle, as the load on the fibril increases the interfacial shear stress at the point of transition increases. Eventually debonding occurs at the point of transition when the magnitude of $\tau$ becomes comparable to the interfacial shear strength.

We now discuss a fibril pullout model for predicting the load in a collagen fibril and the energy required to pull out a fibril. ${ }^{158}$ Consider the embedded fibril end at a distance $Z\left(=z / L_{\mathrm{PO}}\right.$ where $L_{\mathrm{PO}}$ is the embedded fibril
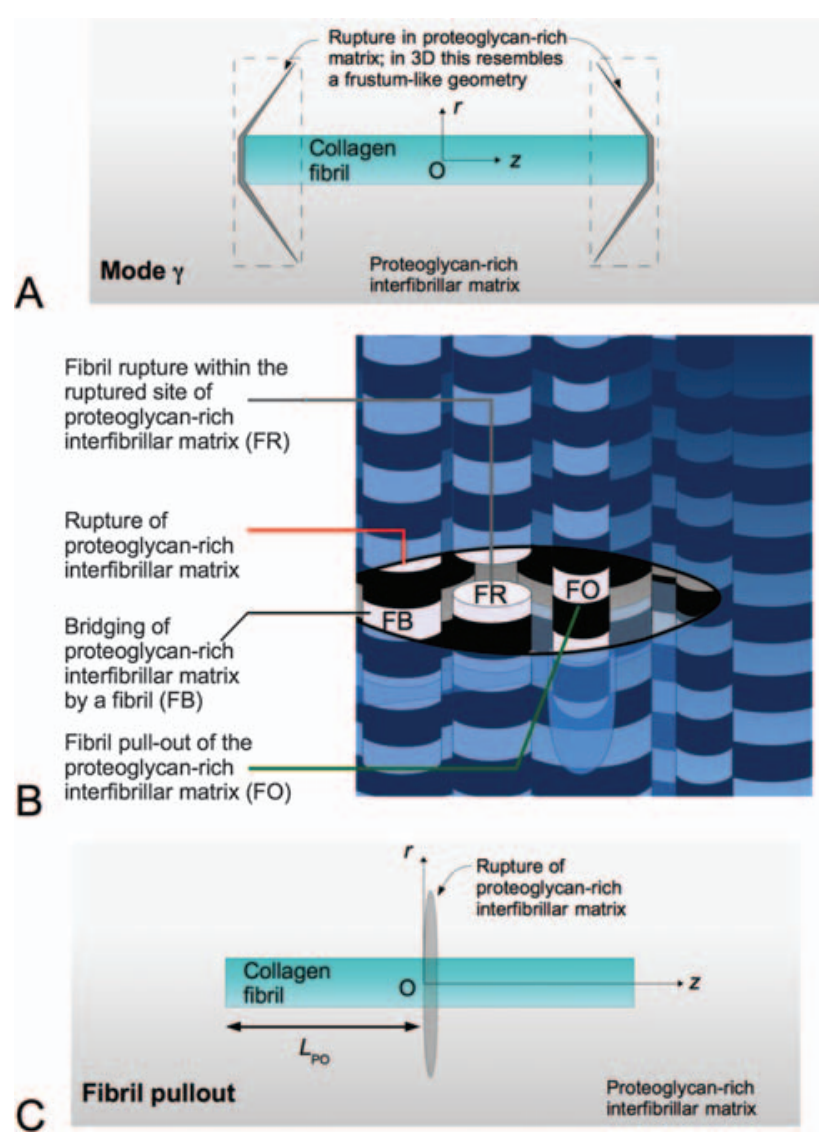

Figure 11. Failure in extracellular matrix (ECM). (A) Schematic of rupture in proteoglycan(PG)-rich interfibrillar matrix in the vicinity of a collagen fibril. The rupture is shown to propagate from the fibril ends (mode $\gamma$ ). The three-dimensional geometry of the rupture resembles a frustum. (B) Schematic of collagen fibrils in ECM, depicting a snap-shot of the various scenarios of failure, namely collagen fibril rupture (FR), pullout (FO), and collagen fibril bridging PG-rich interfibrillar matrix (FB) at the ruptured site of the interfibrillar matrix. Reprinted with permission from [61], $\mathrm{K}$. L. Goh, et al., Bimodal collagen fibril diameter distributions direct age-related variations in tendon resilience and resistance to rupture. J. Appl. Physiol. 113, 1 (2012). ( ) 2012, American Physiological Society. (C) Schematic of fibril pullout at the site of rupture in PG-rich interfibrillar matrix. Symbol $L_{\text {po }}$ represents the embedded length of the fibril; $O$ represents the origin of the cylindrical polar coordinate system where $r$ and $z$ are the radial and axial coordinates. 
length) from the origin, $\mathrm{O}$, which corresponds to the entry point into the PG-rich interfibrillar matrix (Fig. 11(C)). Within the embedded section of the fibril, $\sigma_{z}$ can be determined by solving Eq. (26). This leads to $\sigma_{z}$ expressions of the respective fibril shapes that differ from those derived from the plastic stress transfer problem (Table I) by a negative sign in the factor, $\tau q$. The force applied to the fibril at $\mathrm{O}$ may be written as ${ }^{158}$

$$
F_{\mathrm{PO}}(Z)=\pi r(Z)^{2} \sigma_{z}(Z)
$$

Equation (28) is the general expression for relating $F_{\mathrm{PO}}$ at $\mathrm{O}$ to $\sigma_{z} ; F_{\mathrm{PO}}$ depends on how far the point of application of the force is from the fibril (embedded) end. Another important parameter is the maximum energy to pull a fibril out of the matrix, $G_{\mathrm{PO}}$. We find

$$
G_{\mathrm{PO}}=L_{\mathrm{PO}} \int_{1}^{0} F_{\mathrm{PO}}(Z) \mathrm{d} Z
$$

which expresses $G_{\mathrm{PO}}$ in the form of the work done to pull a length $L_{\mathrm{PO}}$ of fibril embedded in the matrix. ${ }^{158}$ $G_{\mathrm{PO}}$ is an important parameter because it quantifies the energy transfer to overcome frictional forces at the fibril-matrix interface using an energy density parameter defined by

$$
g_{\mathrm{PO}}=G_{\mathrm{PO}} / A_{\mathrm{PO}}
$$

where $A_{\mathrm{PO}}$ is the surface area of the embedded fibril. ${ }^{158}$ For simplicity, only one half of a fibril embedded in the PG-rich matrix is considered so that $L_{\mathrm{PO}}=L_{\mathrm{CF}}{ }^{158}$ (This simple approach is justified because it allows the model to investigate the energy transfer at the extreme of values. Nevertheless the symbol $L_{\mathrm{PO}}$ will be retained throughout this discussion to emphasize the pullout problem.) This area is given by ${ }^{158}$

$$
A_{\mathrm{PO}}=2 \pi \int_{0}^{1} r(Z) \mathrm{d} Z
$$

For each of the fibril shapes, namely uniform cylindrical, straight-tapered, paraboloidal and ellipsoidal ends (Fig. 8(B)), $A_{\mathrm{PO}}$ is obtained by substituting the appropriate expression for $r(Z)$ into Eq. (31). The general expression for the energy transfer at the fibril-matrix interface for the four fibril shapes can be expressed in the form

$$
g_{\mathrm{PO}}=\beta \tau L_{\mathrm{PO}}
$$

where $\beta$ is a numerical factor that depends on the shape of the fibril. ${ }^{158}$ Table II lists the predictions of $g_{\mathrm{PO}} / \tau L_{\mathrm{PO}}$ for the respective fibril shape. Thus, $\beta$ is a constant for the uniform cylindrical fibril; for the fibrils with tapered ends, $\beta$ depends on $q .{ }^{158}$ A plot of $g_{\mathrm{PO}} / \tau L_{\mathrm{PO}}$ versus $q$, reveals that the $g_{\mathrm{PO}} / \tau L_{\mathrm{PO}}$ of tapered fibres increases non-linearly with $q$ at small $q$ values. ${ }^{158}$ (Note, uniform cylindrical fibril yields a constant $g_{\mathrm{PO}} / \tau L_{\mathrm{PO}}$.) Beyond $q=10, g_{\mathrm{PO}} / \tau L_{\mathrm{PO}}$ converges to $0.106,0.126$ and 0.134 , for the straight-tapered, paraboloidal and the ellipsoidal ends, respectively. ${ }^{158}$ We note that these magnitudes of $g_{P O} / \tau L_{\mathrm{PO}}$ are appreciably lower than that of the uniform cylindrical fibril. These results are reasonable: the energy to pull out a tapered fibril is small relative to that of a uniform cylindrical fibril because the energy transfer at the fibril-matrix interface to overcome friction is small. By a simple analogy, less energy is needed to puncture a material using a tapered probe than one with a blunt end and this argument applies for pulling out the probes. Finally, we shall like to point out that the limiting value of $g_{\mathrm{PO}} / \tau L_{\mathrm{PO}}$ in tapered fibrils corresponds to the beginning of a rapidly increasing $A_{\mathrm{PO}}$ as observed in the plots of $A_{\mathrm{PO}}$ versus $q$ of the respective tapered ends, ${ }^{158}$ suggesting that $A_{\text {PO }}$ plays an important role in the energy transfer during fibril pullout. ${ }^{158}$

\section{Fibril Rupture}

Fibrils reinforcing the PG-rich matrix during plastic stress transfer may fragment, producing shorter segments, at the points where the fracture stress is reached. Eventually the fragmentation process terminates because the subsequent fragments generated would not be long enough to take up stress to the level of its fracture stress; the stress transferred to the fibril fragment is insufficient to cause

Table II. Solutions to the fibril pullout problem.*

\begin{tabular}{lcc}
\hline Fibril shape & $g_{\mathrm{PO}} / \tau L_{\mathrm{PO}}$ & $A_{\mathrm{PO}}$ \\
\hline Uniform cylinder & $\frac{1}{3 \pi} \frac{q}{\sqrt{1+q^{2}}}$ & $2 \pi r_{0} L_{\mathrm{PO}}$ \\
Straight-taper & $\frac{16}{5 \pi} \frac{q^{3}}{\left\{1+4 q^{2}\right\}^{3 / 2}-1}$ & $\pi r_{0} \sqrt{r_{0}^{2}+L_{\mathrm{PO}}^{2}}$ \\
Paraboloidal & $\frac{2}{3 \pi} q\left\{1+\frac{q^{2}}{\sqrt{q^{2}-1}} \sin ^{-1}\left(\frac{\sqrt{q^{2}-1}}{q}\right)\right\}^{-1}$ & $\frac{\pi r_{0}}{6 L_{\mathrm{PO}}^{2}}\left\{\left[r_{0}^{2}+4 L_{\mathrm{PO}}^{2}\right]^{3 / 2}-r_{0}^{3}\right\}$ \\
Ellipsoidal & $\pi r_{0}^{2}+\frac{\pi r_{0} L_{\mathrm{PO}}^{2}}{\sqrt{L_{\mathrm{PO}}^{2}-r_{0}^{2}}} \sin ^{-1}\left(\frac{\sqrt{L_{\mathrm{PO}}^{2}-r_{0}^{2}}}{L_{\mathrm{PO}}}\right)$ \\
\hline
\end{tabular}

Notes: *Here, $g_{\mathrm{PO}}$ parameterizes the energy transfer (essentially an energy density quantity) during fibril pullout from the PG-rich matrix; $A_{\mathrm{PO}}$ the surface area of the embedded fibril, $\tau$ the interfacial shear stress; $L_{\mathrm{PO}}$ the fibril embedded length (this is identified with $L_{\mathrm{CF}}$ in the formulation); $r_{0}$ the radius at the fibril centre and $q$ the fibril aspect ratio. 
further fragmentation. ${ }^{162,163}$ In this simplified treatment of fracture, we assume that fracture occurs predictably. In other words, for a given point on the fibril, when the stress at this point reaches the $\sigma_{f}$ then this initiates fibril fracture at this point. A sketch of a graph of $\sigma_{z}$ versus axial distance, $z$, corresponding to the respective fibril fragments (lined up in series) undergoing the plastic stress transfer process, is shown in Figure 10(E). The corresponding graph of $\tau$ versus $z$ is shown in Figure 10(F). Here, we note that: (1) $\sigma_{z}$ increases from the end of a fibril fragment to a peak value at the fibril center; (2) the original fibril possesses a higher peak stress than the short fragments. (We shall explain \#2 in the next paragraph.)

The above findings would require further explanation using the concept of critical length $\left(L_{c}\right)$ of the fibril. ${ }^{143,164,165}$ According to this concept, $L_{c}$ is defined as the minimum length that a fibril must have for the stress at its centre to be equal to $\sigma_{f}$. For a uniform cylindrical fibril, the $\sigma_{z}$ equation (Table I) predicts that $\sigma_{z}$ rises linearly from the ends $\left(Z=0 ; \sigma_{z}=0\right)$, to a maximum at the centre. ${ }^{143}$ In particular, $\sigma_{z}$ reaches a maximum value of

$$
\sigma_{z}(0)=\sigma_{f}=\tau L_{\mathrm{CF}} / r_{0}
$$

At the centre of the fibril. Next, we recall that $\tau$ is constant during plastic stress transfer and acts over the entire fibril. ${ }^{137,140,154}$ For a given tensile stress acting on the fibril, according to Eq. (33), increasing $L_{\mathrm{CF}}$ then increases $\sigma_{z}(0)$ - the increase in $\sigma_{z}(0)$ is expected to continue until the fibril fractures. (As pointed out in previous paragraph, it follows that an increase in $L_{\mathrm{CF}}$ yields a corresponding increase in the magnitude of $\sigma_{z}$, given all things being the same, i.e., $r_{0}$ and $\tau$, in all fragments.) Thus, for effective reinforcement, $L_{C F}$ should be large but less than $L_{c} .{ }^{165,168}$ Although the discussion on the concept of $L_{c}$ is concerned with lending support to the argument of how collagen fibrils provide reinforcement to unidirectional SCTs such as tendons ${ }^{166,146}$ it would interest the reader to know of the wider applicability of the critical length concept to other tissues, namely articular cartilage ${ }^{145}$ and cornea. ${ }^{167}$ In the case of tendons, it is thought that as the tissue (e.g., tendon) grows during the development process, eventually the $L_{c}$ of collagen fibrils must exceed $30 \mu \mathrm{m}$ in order for the tissue to be able to provide optimal mechanical response to an external load. ${ }^{165}$ Unfortunately, the detailed knowledge of the length of collagen fibrils, ${ }^{167}$ let alone $L_{c}$, is still a contentious subject owing to difficulties in isolating collagen fibrils from ECM. ${ }^{81}$ A better understanding of the length of collagen fibril could have important implications on existing models (such as the elastic and plastic stress transfer models described in previous subsections) in which the basis of interfacial shear for enabling collagen fibril stress uptake underpins the assumption that the fibrils are short (discontinuous) in relation to the whole tissue.
Table III. Solutions to the fibril critical length $\left(L_{c}\right)$ and critical volume $\left(V_{c}\right)$ * $^{*}$

\begin{tabular}{lcc}
\hline Fibril shape & \multicolumn{1}{c}{$L_{c}$} & $V_{c}$ \\
\hline Uniform cylinder & $r_{0} \sigma_{f} / \tau$ & $\pi r_{0}^{2} L_{c}=\pi\left\{r_{0}^{3} \sigma_{f} / \tau\right\}$ \\
Straight-taper & $2 r_{0} \sigma_{f} / \tau$ & $(1 / 3) \pi r_{0}^{2} L_{c}=(2 \pi / 3)\left\{r_{0}^{3} \sigma_{f} / \tau\right\}$ \\
Paraboloidal & $(3 / 2) r_{0} \sigma_{f} / \tau$ & $(1 / 2) \pi r_{0}^{2} L_{c}=(3 \pi / 4)\left\{r_{0}^{3} \sigma_{f} / \tau\right\}$ \\
Ellipsoidal & $(4 / \pi) r_{0} \sigma_{f} / \tau$ & $(2 / 3) \pi r_{0}^{2} L_{c}=(8 / 3)\left\{r_{0}^{3} \sigma_{f} / \tau\right\}$ \\
\hline
\end{tabular}

Notes: ${ }^{*}$ Here, $\sigma_{f}$ represents the maximum stress in a fibril before rupture, $\tau$ the interfacial shear stress during plastic stress transfer, and $r_{0}$ the radius at the fibril centre.

Equation (33) can be solved analytically to determine expressions for the $L_{c}$ of fibrils for both uniform cylindrical shape and tapered ends as listed in Table III. ${ }^{168}$ The results have an important and immediate consequence: tapered fibrils have longer $L_{c}$ than uniform cylindrical fibrils given all things being equal, i.e., the value of $r_{0}$. In particular, the $L_{c}$ of a fibril with straight-tapered ends is two times longer than that of a uniform cylindrical fibril. Additionally, by an analogy to engineering composites, ${ }^{143}$ it is believed that the longer the collagen fibrils, the tougher, stronger and stiffer will be the SCT, given all things being the same (i.e., $\left.r_{0}\right) .{ }^{146,165}$ In previous section (Section Elastic stress transfer), it was pointed out that a fibril with tapered ends requires less volume of collagen material to synthesize as compared to uniform cylindrical fibrils, for a given $L_{\mathrm{CF}}$ and $r_{0}$. Advancing this argument further to account for fracture in the context of the critical volume of the fibril, $V_{c}$, which is the volume of a fibril whose length is equal to $2 L_{c}$, the results of $V_{c}$ for the four fibril shapes are determined and listed in Table III. Altogether, these findings are important because they address the critical fracture-related dimensions for the different fibril shapes in terms of the $L_{c}$ and $V_{c}$.

The discussion presented in these subsections has highlighted the key areas of collagen fibril biomechanics, from how a single collagen fibril takes up stress when it undergoes uniaxial extension to how the interaction between the collagen fibril and the PG-rich interfibrillar matrix enables stress to be transferred to the fibril and finally, how collagen fibril fails by pullout and rupture. An immediate and important implication of the stress transfer arguments is the molecular basis underpinning fibril-fibril interaction via the fibril-bound biomacromolecules such as PGs. To the best of our knowledge, the origin of these fibril-bound biomacromolecules remains contentious. The findings that supported the molecular basis have sought to implicate the filament-like GAG side-chains associated with fibril-bound DCN for the role of regulating the fibril-fibril interaction. These findings stems from several sources, namely

(i) structural analyses by electron microscopy, ${ }^{18,50,51,57,169}$ (ii) direct mechanical testing of the elastic stiffness and strength of DCN-DCN interaction using optical tweezers ${ }^{170}$ and DCN deficient SCTs versus controls ${ }^{48}$ as 
well as the dynamic stiffness governing the viscoelasticity of the SCT, ${ }^{169}$ and

(iii) computer modeling of GAGs as interfibrillar bridges. ${ }^{62,171}$

On the other hand, some studies on the SCT viscoelasticity have yielded negative results of the influence of GAGs on the quasi-static stiffness of SCT. ${ }^{172-175}$ Nevertheless, these findings suggest that more studies are needed to uncover the mechanical contribution of other ECM components to fibril-fibril interaction. Moving on, in the next section we discuss the mechanics of collagen fibres leading to tissue extension under uniaxial loading.

\section{Collagen Fibre Sliding Mechanics \\ Non-Uniform Loading of Fibres}

This section is concerned with the loading behavior of collagen fibres. From a structural perspective, variation exists in the structural features, e.g., lengths, diameters and orientations among the fibre bundles of SCTs, ${ }^{91,176}$ particularly the relative orientations of the fibres at the insertion site of the junction between a tendon and muscle ${ }^{98,99}$ and the junction between a ligament/tendon and bone. ${ }^{96,97}$ In previous section (Section Collagen fibre), we have pointed out that if the collagen fibre length spans the tissue length, this implicates that tissue extension is attributed to fibre extension. However, if the fibres span only the midsection of the tissue but a proportion of these terminate at the junction of the adjoining tissue (e.g., bone), tissue extension could be explained by fibre sliding. FE analyses of the biomechanical models of anterior cruciate ligament have predicted that these structural variations contribute to the variation in the stress uptake along the fibre bundles ${ }^{91,95}$ in other words collagen fibre bundles in the SCT are nonuniformly loaded during joint motions. The basis of nonuniform loading may be attributed to the simultaneous 'stiffening' of the fibres of the posterolateral bundle and relaxation of the fibres of the anteriomedial bundle during hyperextension. ${ }^{177}$ Consequently, the regions of high stress concentration in the anterior cruciate ligament are found at the attachment sites and the presence of stress concentration here could be a contributory factor to tissue rupture, in accordance with observation of rupture in the ligament near the femoral attachment when the knee is in hyperextension. ${ }^{155,178}$

We have not come across any report describing the experimental study of the influence of the relative displacements of the fibres (at the insertion sites) on the stress uptake by the individual collagen fibres. A starting point to closing the gap in this area is to investigate the stress distribution along a collagen fibre, taking into consideration its interactions with adjacent fibres and the attachment sites. This could be realized by adapting an approach, known as the local strain measurement, to evaluate the contribution of collagen fibre sliding and extension to the overall tissue extension. ${ }^{8,9}$ The method employs cells that are present along the collagen fibre as strain markersthe underlying assumption in these experiments is that each cell is able to bind to the collagenous matrix. ${ }^{87,179}$ By monitoring these cells during loading (Fig. 12(C)) the strains contributed by each fibre and the relative (sliding) displacement between fibres can be determined., ${ }^{8,9}$ Overall, local strain within a collagen fibre increases with applied strain (Fig. 12(A)). Interestingly, the local strain appears to plateau out (at about 0.015 of the applied strain; Fig. 12(A)) as the applied strain increases up to 0.08 (i.e., in the elastic region; Fig. 4(A)). The relative displacement (sliding) of collagen fibre bundles also increases with applied strain (Fig. 12(B)). However, unlike the local strain within the fibre, the relative displacement increases (up to $5 \%$ of the applied displacement; Fig. 12(B)) without
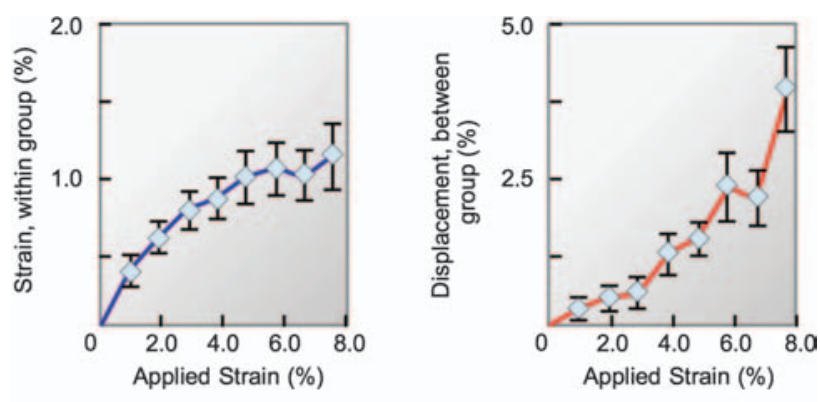

A

B

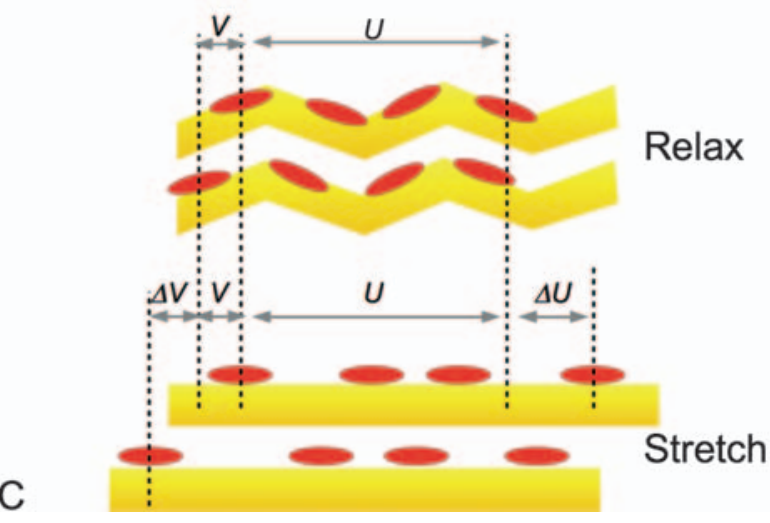

Figure 12. Local strain measurements in extracellular matrix. (A) Graph of the within-group strains versus applied strain. (B) Graph of the between-group displacements (expressed as a percentage of the overall fascicle displacement for all fascicles tested) versus the applied strain. Reprinted with permission from [8], H. R. C. Screen, et al., Local strain measurement within tendon. Strain 40, 157 (2004). @ 2004, John Wiley and Sons. (C) Schematic of cells on collagen fibres, depicting the location of the cells in relation to other cells on the same collagen fibre and on different collagen fibre. Top panel, when the tissue is in a relax state; bottom panel when a tissue is subjected to an external load. For the purpose of illustration the cells are numbered. Figure adapted with permission from [9], H. R. C. Screen, et al., An investigation into the effects of the hierarchical structure of tendon fascicles on micromechanical properties. Proc. IME H. J. Eng. Med. 218, 109 (2004). (C) 2004. Displacement between group $=[100 \Delta V / \Delta L]$; strain within group $=[100 \Delta U / U]$. 
appearing to plateau out at large applied strain. This suggests that the extension of the tissue up to the elastic region is largely contributed by the shear action between collagen fibres. ${ }^{8,9}$ Additionally, it is reported that collagen fibre sliding behaviour is reversible for as long as the applied strains do not exceed a value of $0.05 .^{8,9}$ Although the two parameters local strain and relative sliding displacements are not directly comparable, the issue concerning which of the two mechanisms is a major contributor to the tissue extension is debatable. Nevertheless, these findings clearly indicate that both mechanisms are responsible for tissue extension. ${ }^{8,9,94}$ Additionally collagen fibre sliding may also be a contributory factor to the increase in tissue length during growth. ${ }^{94}$

\section{Role of Microscopic Crimps}

The mechanics of collagen fibres sliding at low strains (0.03-0.04; Fig. 12(A)) is of interest as these strains encompass the toe-to-heel region where the SCT undergoes decrimping as it stretches. Here, the upper limit of the region is consistent with quantitative measurement of the extinction of crimp pattern, which shows up as bands in images taken using optical coherent tomography, in tendons from rat tail at strain $\approx 0.03 .{ }^{180}$ Crimp is thought to play two important roles, namely as a shock absorber on initial loading and for elastic recoil. ${ }^{181-184}$ It is thought that crimp is a consequence of the contraction of cells (e.g., fibroblasts) bound on collagen fibres, thus creating a pull force on the fibres. ${ }^{185}$ This pulling force is facilitated by the large differential stiffness between the collagen fibres and the interfibre matrix; the larger the relative stiffness ratio of collagen to PG-rich matrix the more pronounced is the crimp. ${ }^{185}$ Injured tissues exhibit sub-optimal mechanical response to an external load and this could be attributed to crimp disruption arising from the physical injury. ${ }^{186}$ If cells play an important role in the formation of crimps by pulling on the fibres as suggested in a report, ${ }^{185}$ then crimp disruption could also be attributed to the presence of a lower cell density because a large number of cells would have died from avascularity at the ruptured sites. Altogether these factors suggest that crimps have important implications for tissue healing and regeneration. ${ }^{185}$

From the cell perspective, the disruption of crimps in injured tissue could in turn affect the mechanotransduction pathways. Whereas the mechanical signals of these pathways are thought to be sensitive to the local environment, ${ }^{21}$ particularly the variation in the strain at any point along the collagen fibre and the relative displacement of the fibres, ${ }^{8,9,85,86}$ the magnitude of the local strain and the displacement of the fibres in turn depend on the local mechanical properties, e.g., stiffness, resilience of the fibre. How the cell may be mechanically stimulated to undergo deformation depends on how these properties influence the key cellular processes via interaction with integrin receptors. ${ }^{187}$ Nevertheless, the mechanisms by which mechanical forces direct cellular biochemical and molecular responses remain undefined. In particular, further investigations are needed to link the specific processes, namely fibre decrimping, fibre extension and fibrefibre sliding during uniaxial extension of the SCT, to the mechanotransduction pathways.

\section{Origin of Fibre-Fibre Sliding}

Although the shear behaviour of the interfibre matrix is believed to play an important role in the mechanics of collagen fibre sliding, the identity of the underlying ECM components responsible for the shearing behavior is still not clear. Removal of GAG side-chains by enzyme Chondroitinase $\mathrm{ABC}$, which was intended to investigate the effects at the level corresponding to collagen fibril, led to significant swelling of collagen fibrils and the surrounding matrix. However, it yielded no appreciable effect on collagen fibre extension and fibre-fibre sliding. ${ }^{85,86}$ This suggests that fibril-associated PG GAGs on the peripheral of the fibre play no part in anchoring the fibre with the interfibre matrix. The swelling of fibrils and the surrounding matrix is attributed to water gelation as demonstrated by MD simulation ${ }^{59}$ - water plays an important role in the viscoelasticity of the SCT right down to the molecular level by mediating the shearing between TC molecules. ${ }^{114}$ Although the Hyp residues in the Gly-ProHyp amino acid sequence (see Section Fibrillogenesis) are able to form hydrogen bonds with water molecules, ${ }^{59,114}$ binding between the peptides and water molecules are dominated by electrostatic interactions and van Der Waals interactions. ${ }^{59}$

\section{Elasticity and Fracture Toughness of Structural Units \\ Scale-Dependent Mechanical Properties}

The intent of this section is to discuss the mechanical properties of the structural units of the respective levels of the hierarchical architecture and the interconnection of the mechanical properties from a molecular level upward. Of course the mechanical properties of whole tissues have been well reported. However the arguments for linking the mechanical properties of ECM structural units at the respective levels to those of whole tissue seems to be less established. We begin our argument by referring the reader to the stress-strain curve of the whole tissue in relation to that of collagen fibril and TC molecule (Fig. 13(A)). ${ }^{107}$ The results were obtained from simultaneous mechanical testing and XRD to measure the mechanical response of the two structural units, TC molecules and collagen fibrils in tendons (rat tail) from initial loading until the whole tissue reached a strain of about $0.06 .^{107}$ The profile of the stress-strain curve of the whole tissue is typical of those reported elsewhere, e.g., Figure 4(A) (the strain of 0.06 lies in the elastic region). Of note the toe-to-heel region is attributed to the continuous process 
involving the straightening of the microscopic crimps (see Section Microscopic crimps). Since energy is needed to straighten the crimps, these crimps act as a shock absorber against undue damage to the collagen fibre bundle (see Section Role of microscopic crimps). At higher loads, when the crimps are extinguished, extension proceeds elastically (this occurs at a strain of 0.02). At any given strain point up to 0.06 , the gradient (a measure of the stiffness) of the stress-strain curve of the structural units increases as the level of the hierarchical architecture decreases. In other words, the TC molecule possesses the largest stiffness; the stiffness of collagen fibril lies in between that of the TC molecule and whole tissue. From the perspective of the strain parameter, for a given stress the strain value of the corresponding stress increases as the level of the hierarchical architecture increases. Thus, this suggests that the TC molecule is least extensible (compared to collagen fibril and whole tissue). We note that the ability of the collagen fibril to deform by extension under load lies somewhat between that of the TC molecule and the whole tissue.

We have illustrated the different mechanical responses of the structural units at two levels, comparing them with those from whole tissue, to suggest the trend in the magnitudes of the mechanical properties with decreasing level from whole tissue to the molecular level. To investigate the possible scale-dependent mechanical properties we review the findings from literature on uniaxial extension studies. Here we focus on the following mechanical parameters termed simply as stiffness, strength, extensibility and toughness. To ensure consistency in parameterizing the mechanical properties, we consider the studies that have investigated the mechanical properties in the following way:

(1) the tangent to the stress-strain curve at any point between the toe-to-heel and the yield point parameterizes stiffness;

(2) the maximum stress parameterizes strength;

(3) the maximum strain parameterizes extensibility;

(4) the strain energy density to rupture parameterizes toughness.

The findings from the literature on the stiffness, strength, extensibility and toughness of whole tissue, fascicles, collagen fibrils and TC molecule are listed in Table A1 (see Section Appendix). We have chosen to represent the numerical data from mean values, or from values of individual specimens or by order of magnitude estimates from stress-strain relationships, depending on what was reported. In particular, order of magnitude estimates from stress-strain relationships were carried out when the magnitudes of the respective mechanical parameters were not reported explicitly in the study. For estimating stiffness, we note that stiffness is of the order of strength divided by the strain at maximum stress. This seems reasonable when the toe-to-heel region is not appreciably visible; otherwise the gradient from the linear region of the stress-strain curve would be evaluated to estimate the stiffness. For estimating the toughness, we note that toughness is identified with one half of the product of strength and extensibility. In particular, where the estimates concern the TC molecule, we note that (1) strength is of the order of the ratio of $F$, the force generated in the TC molecule (Eq. (2)), to $\alpha_{\text {mol }}\left(=\pi r_{\mathrm{TC}}^{2}\right)$, where $r_{\mathrm{TC}}$ is set equal to a practical value of $0.28 \mathrm{~nm} ;{ }^{14,15}$ it seems reasonable to adopt this value because this is the smallest value that we could find so far that can account for the largest possible strength, (2) extensibility is of the order of the ratio of the axial displacement of the molecule to $L_{\text {con }}$, the molecular contour length, which is $\approx 310 \mathrm{~nm}^{14,15}$ (see Section Tropo-collagen mechanical properties). In our simplified treatment of the numerical data from the literature, we have made no distinction in the data from specimens with regards to age groups, anatomical types, species and strain rates. Inevitably, this leads to wide variability in the magnitudes of the respective mechanical properties at each level. However, we find that the variability of the respective mechanical properties at each level paled into insignificance compared to the variability in the mechanical properties across different length scales. To better account for the scale-dependency in the mechanical properties, we use a normalized parameter to represent the mechanical property of the respective levels. To this end, we determine the average values of the mechanical properties for stiffness, strength, extensibility and toughness of the respective levels base on the values taken from all the studies listed in that level in Table A1. The normalized mechanical properties for stiffness, strength, extensibility and toughness of the respective levels are identified with the ratio of the corresponding average value of the mechanical property to a predetermined theoretical value of the corresponding mechanical property of the TC molecule. Here, the theoretical values for stiffness, strength, extensibility and toughness of the TC molecule may be determined from the WLC model (Eq. (15)). Considering that stress is identified with the ratio of $F$ to $\alpha_{\text {mol }}$ and strain is identified with the ratio of axial displacement to $L_{\text {con }}$, by evaluating Eq. (15) we obtained a plot of the theoretical stress versus strain of the TC molecule as shown in Figure 13(B). We then estimated the theoretical strength of the TC molecule by setting it equal to 0.99 of the strain at unity; with this practical value, it seems reasonable to take the theoretical extensibility $=0.99$. The theoretical stiffness is set equal to the gradient over the linear region. The theoretical toughness is identified with the area under the theoretical stress-strain curve of the TC molecule from strain $=0$ to 0.99 .

Figures 13(C) to (F) show bar charts of normalized stiffness, strength, extensibility and toughness versus the respective structural units. We then find that the TC molecule yields the largest normalized stiffness. One may be tempted to generalize that the lower levels of the hierarchical architecture of ECM are stiffer than the higher 

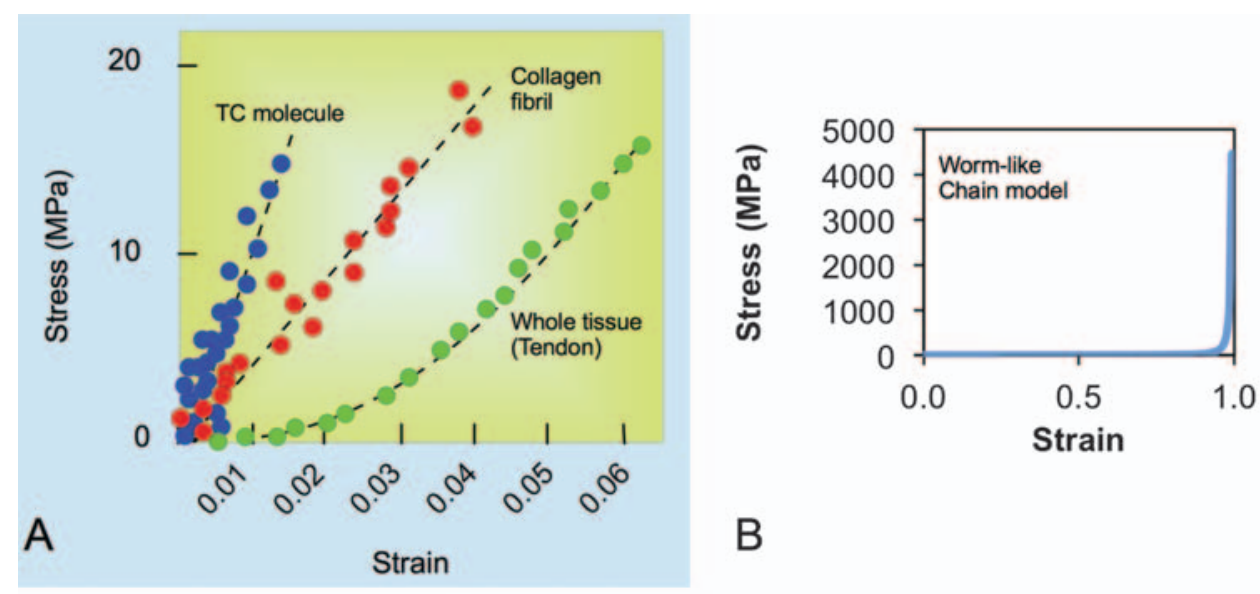

B
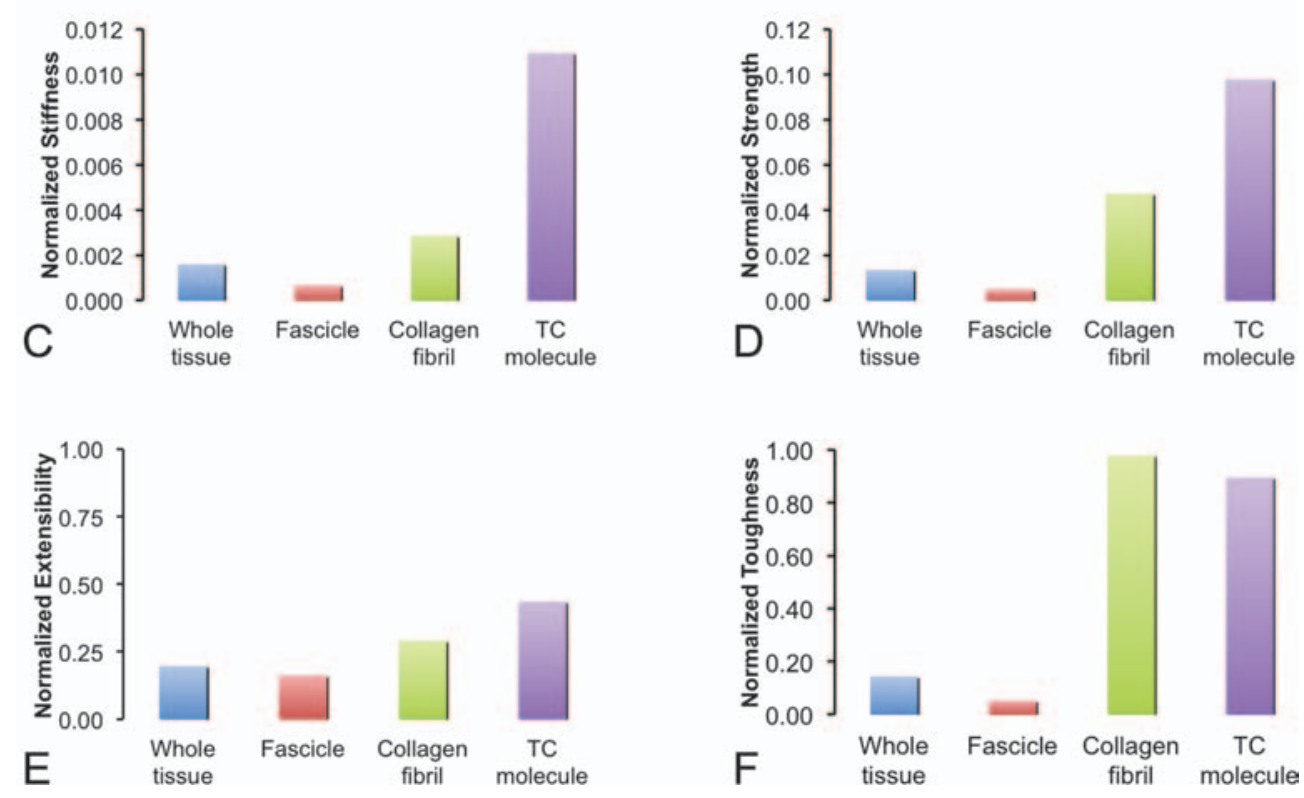

Figure 13. Scale-dependent mechanical properties. (A) Graphs of stress versus strain for tropo-collagen (TC) molecule, collagen fibril and tendon. The results for the TC molecule and collagen fibril were obtained from simultaneous mechanical testing and $\mathrm{X}$-ray diffraction experiment. For the TC molecule, the results were obtained by measuring the strain corresponding to changes in the breadth of the meridional reflections (distance between neighbouring amino acids). For the collagen fibril, the results were obtained by measuring the changes in the $D$ period. Reprinted with permission from [107], N. Sasaki and S. Odajima, Elongation mechanism of collagen fibrils and force-strain relationship of tendon at each level of structural hierarchy. J. Biomech. 29,1131 (1996). ( 1996 , Elsevier. (B) Graph of the theoretical stress versus strain of TC molecule derived from the worm-like-chain model. Bar charts of normalized mechanical properties, namely $(C)$ stiffness, $(D)$ strength, $(E)$ extensibility and $(F)$ toughness versus the structural unit of the respective levels of the hierarchical architecture of extracellular matrix.

levels. ${ }^{12,133}$ However, while this holds for TC molecule versus collagen fibril and fascicle, it appears that the normalized stiffness of whole tissue is somewhat larger than that of fascicle. From an experimental perspective, we speculate that undue damage could have been inflicted to the fascicles during the process of isolating and preparing the fascicles for mechanical testing, leading to a lower magnitude in the mechanical properties. ${ }^{188}$ The damage could be inflicted in the collagen fibrils, manifesting as microscopic lacerations along the fascicles that may not be appreciably visible under a microscope, when teasing the fascicles out from the tendon. ${ }^{188}$ Under these circumstances, it is clear that the damage would have an influence on all mechanical properties. Incidentally, the larger estimates of the mechanical properties, namely stiffness, strength, extensibility and toughness, for whole tissue as compared to fascicle are also consistent with those reported for stress at failure, strain at failure and stiffness. ${ }^{93}$ Moving on, we find that the TC molecule also yields the largest normalized strength and extensibility. Of note, in the case of extensibility it may also be argued that the differences in the normalized extensibility values across the different levels are only marginal. Additionally, the findings for extensibility appears somewhat surprising 
in that it suggests that large extensibility is a consequence of a combination of high stiffness, strength and toughness. With regards to toughness, it turns out that the collagen fibril yields the largest normalized toughness $(\approx 0.98)$ but the difference may be regarded as marginal (TC molecule trails behind at $\approx 0.90$ ) when one compares these values to those of whole tissue $(\approx 0.14)$ and fascicle $(\approx 0.05)$. Nevertheless, the comparative analysis of the mechanical properties from whole tissue to TC molecule reveals that the mechanical properties are scale-dependent.

\section{Hydrogelator Model}

Let us examine more fundamentally how the variations in the mechanical properties of the structural units reconcile with the tissue mechanical properties. As a special case, we consider the stiffness parameter for the purpose of our discussion. For simplicity and for reasons that will become clear, we develop our argument based on stiffness that reflects normal physiological condition, whereby water is present in large proportion in ECM; water serves to hydrate the structural units and support the transport of nutrients from the capilliary vessels to the cells via ECM. However, water also plays an important role in regulating the mechanical properties of the collagen-based structural units. $^{45,133}$ (Thus, we shall also refer to the extreme condition corresponding to dehydration to make useful comparison, where needed.) We recall the amino acid sequence, Gly-Pro-Hyp, as the molecular motif for a TC molecule (see Section Fibrillogenesis). Here, the hydrophobic region is found on Pro and the hydrophilic region is found on Hyp (attributed to the hydroxyl group). ${ }^{59}$ Since the presence of the hydrophobic and hydrophilic regions on collagen satisfies the characteristic of amphiphilicity of a hydrogelator, ${ }^{59}$ we find that in fact, the physical combination of the two components, namely water and collagen, forms a hydrogelator system. Making use of the rule-of-mixture for modelling the stiffness of SCT, ${ }^{61,167,189}$ accordingly, at the level corresponding to collagen fibril the stiffness, $E_{\mathrm{CF}}$, depends on the two components, namely TC molecules and the hydrated (gel-like) matrix surrounding the TC molecules. ${ }^{59}$ We thus predict that to order of magnitude,

$$
E_{\mathrm{CF}}=E_{\mathrm{TC}} V_{\mathrm{TC}}+E_{\mathrm{H}_{2} \mathrm{O}} V_{\mathrm{H}_{2} \mathrm{O}}
$$

where $E_{\mathrm{TC}}$ and $E_{\mathrm{H}_{2} \mathrm{O}}$ are the respective stiffness of the TC molecule (see Section Tropo-collagen mechanical properties) and the gel-like matrix surrounding the TC molecule $\left(E_{\mathrm{TC}}>>E_{\mathrm{H}_{2} \mathrm{O}}\right)$ and $V_{\mathrm{TC}}$ and $V_{\mathrm{H}_{2} \mathrm{O}}$ are respectively the volume fraction of the TC molecule and the gel-like matrix (i.e., $\left.V_{\mathrm{TC}}+V_{\mathrm{H}_{2} \mathrm{O}}=1\right)$. For a dehydrated fibril, $E_{\mathrm{CF}} \approx E_{\mathrm{TC}}$ because $V_{\mathrm{TC}} \approx 1\left(V_{\mathrm{H}_{2} \mathrm{O}} \approx 0\right)$. However, for a hydrated fibril, assuming that $V_{\mathrm{H}_{2} \mathrm{O}}>>V_{\mathrm{TC}}$, it follows that water in the local environment could contribute to reducing the magnitude of the term, i.e., $E_{\mathrm{TC}} V_{\mathrm{TC}}$, substantially. Consequently, this reduces the magnitude of $E_{C F}$ and we find $E_{\mathrm{TC}}>$ $E_{\mathrm{CF}}$. Going up to the next level, i.e., fascicle, we apply a similar argument for the stiffness, $E_{\mathrm{FAS}}$, of the hydrogelator comprising collagen fibrils and the PG-rich (hydrated) interfibrillar matrix. We thus predict to order of magnitude that

$$
E_{\mathrm{FAS}}=E_{\mathrm{CF}} V_{\mathrm{CF}}+E_{\mathrm{PG}} V_{\mathrm{PG}}
$$

where $E_{\mathrm{CF}}$ and $E_{\mathrm{PG}}$ are the respective stiffness of the collagen fibril and the PG-rich interfibrillar matrix $\left(E_{\mathrm{CF}} \gg\right.$ $\left.E_{\mathrm{PG}}\right)$ and $V_{\mathrm{CF}}$ and $V_{\mathrm{PG}}$ are respectively the volume fraction of the collagen fibril and the PG-rich interfibrillar matrix (i.e., $V_{\mathrm{CF}}+V_{\mathrm{PG}}=1$ ). The situation corresponding to a dehydrated fascicle leads to $E_{\mathrm{FAS}} \approx E_{\mathrm{CF}}$ because $V_{\mathrm{CF}} \approx 1$ $\left(V_{\mathrm{PG}} \approx 0\right)$. However, for a hydrated fascicle, assuming that $V_{\mathrm{PG}}>V_{\mathrm{CF}}$, it follows that water in the local environment could contribute to reducing the magnitude of the term, i.e., $E_{\mathrm{CF}} V_{\mathrm{CF}}$, substantially. Under these circumstances, this reduces the magnitude of $E_{\mathrm{FAS}}$ and we find $E_{\mathrm{CF}}>E_{\mathrm{FAS}}$. Extending the hydrogelator argument to the case of fascicle versus whole tissue, we find that the stiffness of the hydrogelator, $E_{\mathrm{WT}}$, is regulated by two components, namely the fascicle and the hydrated intrafascicular matrix. We thus predict to order of magnitude that,

$$
E_{\mathrm{WT}}=E_{\mathrm{FAS}} V_{\mathrm{FAS}}+E_{\mathrm{IFM}} V_{\mathrm{IFM}}
$$

where $E_{\mathrm{FAS}}$ and $E_{\mathrm{IFM}}$ are the respective stiffness of the fascicle and the hydrated intrafascicular matrix $\left(E_{\mathrm{FAS}} \gg\right.$ $\left.E_{\mathrm{IFM}}\right)$ and $V_{\mathrm{FAS}}$ and $V_{\mathrm{IFM}}$ are respectively the volume fraction of the fascicle and hydrated intrafascicular matrix (i.e., $\left.V_{\mathrm{FAS}}+V_{\mathrm{IFM}}=1\right)$. Since one usually finds densely packed parallel arrays of fibre bundles in hydrated SCTs, when observation is carried out under a microscope (Fig. 1(B)), it may not be unreasonable to assume that the volume of the hydrated intrafascicular matrix is appreciably smaller than that of the fascicles. Under this circumstance, to order of magnitude we may identify $E_{\mathrm{WT}}$ with $E_{\mathrm{FAS}}$. In fact, this is not an unrealistic assumption given the normalized stiffness of whole tissue is, within the margin of experimental errors, only marginally larger than that of fascicle when one compares the normalized stiffness from molecular level upwards.

To wrap up the discussion, according to the predictions from the hydrogelator model, the stiffness of the structural units of the respective level could be much larger than whole tissue (or fascicle). This simple analysis suggests that water could be a major contributory factor to regulating the mechanical properties of the structural units from molecular level to fascicle level. In accordance with the rule of mixture for strength and extensibility, ${ }^{164}$ a similar argument may be adapted for reconciling the variation in the strength and extensibility in the structural units with that of whole tissue. Although this simple argument may not reproduce the complexity of the ECM organization in terms of the specific structural feature, it nevertheless captures a fundamental aspect of the hierarchical architecture of ECM that indicates that the magnitude of the mechanical properties could increase with decreasing level. 


\section{The Way Forward}

\section{ECM Mechanics Framework}

In envisioning the goal to a complete understanding of the structure-function relationship of ECM, it may be important to establish a framework for ECM mechanics that describes the mechanisms of stress uptake in the structural units reinforcing ECM at the respective levels of the hierarchical architecture in a consistent manner. Such a framework could allow for comparison of these mechanisms and for making predictions concerning the interconnection of these mechanisms that can also assist in the identification of new mechanical pathways. The framework will underscore the integrative approach that draws on findings from various disciplines using a wide range of experimental, computer modeling and analytical techniques to address the respective length scales from molecular level upwards.

A schematic of such a framework is shown in Figure 14. The starting point of the schematic is the macroscopic mechanical response of a SCT, represented by a stressstrain curve. The curve is divided into five regions corresponding to the respective mechanical processes, namely toe-to-heel, elastic deformation, yielding, plastic deformation and rupture. Each process connects to the processes in the next level. Here we have associated the next lower level with collagen fibre. The processes occurring

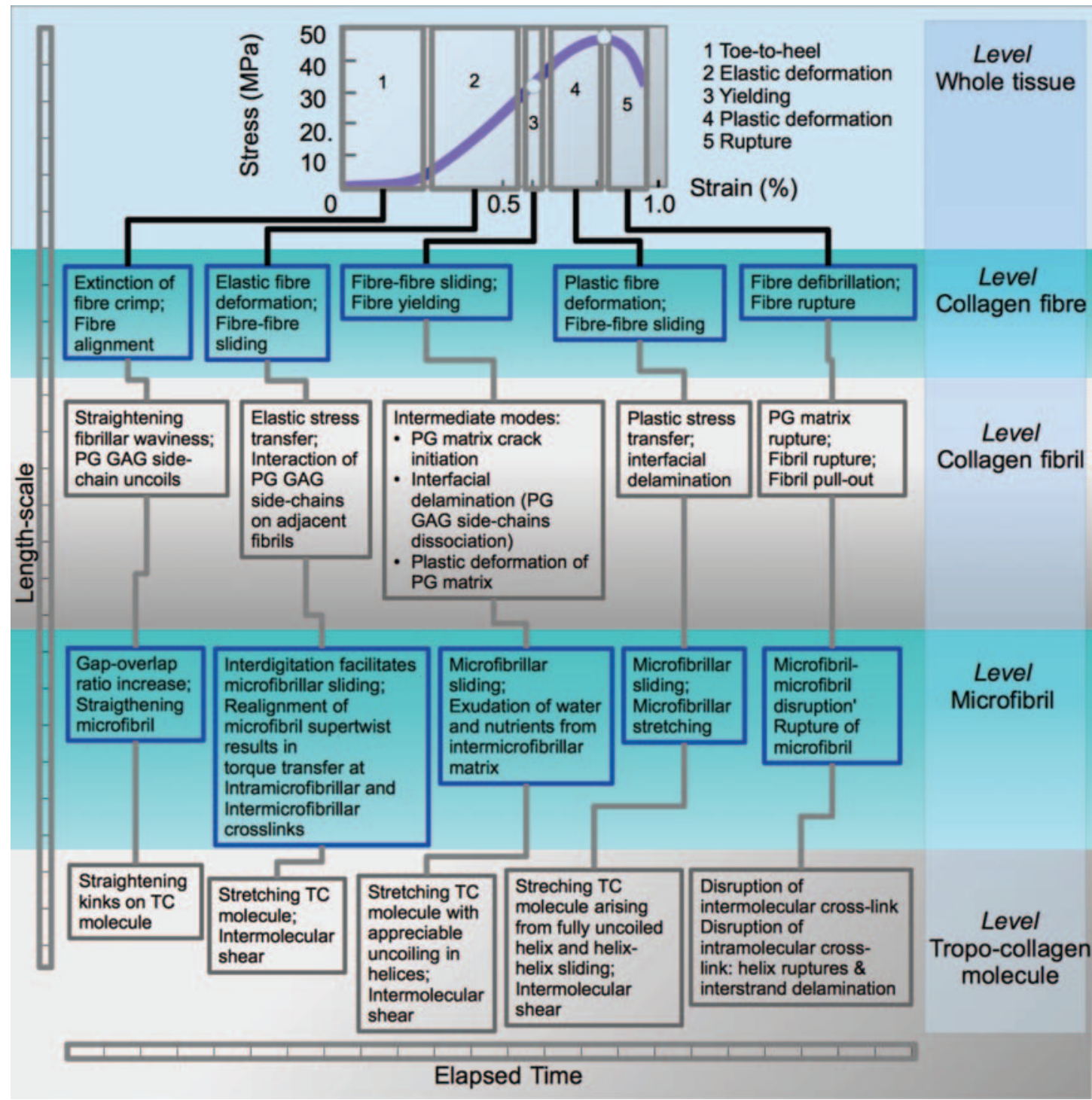

Figure 14. A framework for the mechanics of extracellular matrix (ECM) in soft connective tissue. The framework integrates the findings from low-dimensional to macroscopic studies, to describe the mechanisms of stress uptake in ECM structural units at the respective levels of the hierarchical architecture. The aim of the framework is both to allow for comparison and to make prediction of the interconnection (mechanical pathway, highlighted by thick gray lines) of the mechanisms that can also assist in the identification of new mechanical pathways. PG, proteoglycan; GAG, glycosaminoglycan; TC, tropo-collagen. 
at the collagen fibre level involve decrimping, elastic deformation by fibre-fibre sliding, fibre yielding, plastic deformation, and fibre defibrillation or rupture. Each of these processes in turn connects to corresponding processes at the collagen fibril level. The processes occurring at the collagen fibril level are the extinction of fibril waviness, uncoiling of the fibril-associated PG GAG side-chains, elastic stress transfer (accompanied by the interaction of the PG GAG side-chains on adjacent fibrils), intermediate modes (PG-rich interfibrillar matrix cracks, partial delamination of interface between PG and fibril, PG-rich interfibrillar matrix undergoes plastic deformation), plastic stress transfer (with complete delamination of interface between PG-rich matrix and fibril), rupture of PG-rich interfibrillar matrix, fibril rupture and fibril pullout. Again, each of these processes in turn connects to processes at the microfibril level. The processes occurring at the microfibril level are the straightening of microfibrils (increasing gap-overlap ratio), microfibrillar sliding and realignment of microfibril from its supertwist, exudation of water and nutrients from intermicrofibrillar matrix, microfibrillar stretching, disruption of microfibrilmicrofibril interactions and microfibril rupture. Finally, each of these processes in turn connects to processes at the TC molecular level. These molecular processes are the straightening of kinks on the TC molecule, TC molecular stretching (involving axial deformation of the backbone, uncoiling the helices and helix-helix sliding) and intermolecular shear (nucleation of slip-pulse), and disruption to the intramolecular cross-links and intermolecular cross-links.

An important and immediate consequence of this framework is the prediction of mechanical pathways. On Figure 14, the mechanical pathways are illustrated using lines connecting from one process to the next. For convenience we have named these using the terms designated for the five macroscopic processes. Thus, we have the

(1) toe-to-heel,

(2) elastic deformation,

(3) yielding,

(4) plastic deformation and

(5) rupture pathways.

For the purpose of this discussion we highlight the elastic deformation pathway. At the macroscopic level, this involves the elastic deformation of the SCT. (As the name suggests, upon removal of the applied load, the tissue is expected to relax to its original state.) Extending the pathway to the collagen fibre level, we find that this involves fibre-fibre sliding as well as fibre stretching. Extending the pathway to the collagen fibril level, we find that this involves the elastic stress transfer mechanism whereby both fibril and PG-rich interfibrillar matrix deform elastically and stress transfer occurs at the interface via interactions between the PG GAGs on adjacent fibrils. Extending the pathway to the microfibrillar level, we find that the processes regulating microfibrillar sliding are also involved in regulating the realignment of the microfibril supertwist in the direction of the load acting on the fibril. Finally, extending the pathway down to TC molecular level reveals that the basis of elastic deformation involves the stretching of TC molecules-this is manifested as the deformation of the molecule backbone chains-and the intermolecular shear (by nucleation of slip-pulse). One key feature of the framework is that it enables the respective mechanical pathways to be defined uniquely (through the interconnection of the various levels) and in a consistent manner that allows for comparison among the pathways. For instance, as we compare one pathway against another, we find that while several pathways share similar molecular mechanisms, e.g., stretching and intermolecular shear, the differentiation become more apparent as one interconnects up the levels to the macroscopic processes.

In principle, such a framework could allow for the eventual identification of all possible gaps in the knowledge that are fundamental to understanding the complex ECM. Of immediate concern is a paucity of experimental data to guide our understanding of the mechanisms of TC molecular interaction, microfibril-microfibril interaction, fibril-fibril interaction and fascicle-fascicle interaction. In particular, there are many studies on the structural aspects of fibril-associated PGs but little emphasis has been given to the modeling of the functional aspects of these PGs for fibril-fibril interaction. If fibril-fibril interaction studies are envisioned in the near future, the key parameters for describing the organization (e.g., packing distribution) of collagen fibrils, namely the fibril-fibril lateral (side-to-side) separation and fibril-fibril overlap distance must be addressed. For a comprehensive analysis of the parameters for describing fibril-fibril interaction, this would include studies on the sensitivity of the fibril axial stress uptake during elastic stress transfer, plastic stress transfer and during rupture to variation in the fibril-fibril lateral (side-to-side) separation and fibril-fibril overlap distance parameters. Recognition of such a gap then allows for further investigation to be carried out by experiment. Additionally, the insights gain from integrating the findings within this framework could aid in the design of new models to provide for quantifiable as well as qualitative predictions. The models would be used for analyzing complex interactions before further experiments are to be carried out. One possible model is the multiscale model ${ }^{72}$ which is discussed in the next section.

\section{Multiscale Model}

In the previous subsection, we have pointed out that the insights gain from integrating the findings within the ECM mechanics framework could aid in the design of new models. In principle, this would require a multiscale model of 
ECM to link the mechanical properties of the lower structural units to the macroscopic mechanical properties of the whole tissue. As a simple case, we shall discuss the Annovazzi and Genna multiscale model. ${ }^{72}$

The Annovazzi and Genna model relies on an engineering constitutive approach to predict the stress-strain relationship of SCTs. The underlying implementation addresses the SCT as a parallel arrangement of structural (fibrous) units for the respective levels. In this simplified treatment, to deal with a 'recurring' structure, each unit describes a parallel arrangement of sub-units, and so on until we arrive at the level corresponding to the TC molecule. According to this argument, we see that the mechanical properties of the TC molecule are crucial inputs for the multiscale model. For modelling the mechanics of the TC molecule, to order of magnitude, we identify the axial force generated in the molecule with the $F\left(u_{m}\right)$ (i.e., a function of $u_{m}$ ) of the WLC model (Eqs. (15) and (16)). For the Annovazzi and Genna model, all structural units are assumed extensible (and crimped) at the start. ${ }^{72}$ Consequently, at the molecular level we can assume that the intrinsic elastic deformation predominates (over the entropic deformation). Accordingly, at the start of the stretching process, the length of a structural unit in the unstretched (and uncoiled) state is a random variable, with a predetermined probability distribution function. By integrating over the statistical distribution of the unstretched (and uncoiled) length of all structural units, at all levels, a stress-strain curve can be derived for the tissue (or for any structural unit of any level). Consider a model of $n$ levels whereby the lowest level is designated $n$. For example, if we consider the levels listed in Figures 13(C)-(F), we find that the whole tissue, fascicle, collagen fibril and TC molecule correspond to level $i=1,2,3$ and 4, respectively $(n=4)$. The deformation of the structural unit at level $i$ is expressed as

$$
u_{i}=u_{1}+L_{\text {bundle }}\left(1-\Pi_{j=1}^{i-1} x_{j}\right)
$$

where $x_{j}$ is the ratio of the unstretched length of the $i$ th structural unit, to the length of its projection along the structural unit axis, $u_{1}$ is the axial displacement of the structural unit at the upper-most level (i.e., whole tissue), and $L_{\text {bundle }}$ the unstretched length of the bundle. The corresponding stress generated in the bundle at the $i$ th level is expressed as

$$
\begin{aligned}
\sigma_{i}= & \frac{K_{\mathrm{TC}}}{\alpha_{\mathrm{TC}}} u_{1} P_{n}\left(1-\int_{j=1}^{u_{1}-E\left(\bar{u}_{n}\right) P_{N}\left(u_{1}\right)} p_{\text {fail }}(u) \mathrm{d} u\right) \\
& \times \Pi_{j=n(i<n)}^{i+1} P_{j}
\end{aligned}
$$

where $K_{\mathrm{TC}}$ is the elastic stiffness (force/displacement) of the TC molecule, $\alpha_{\mathrm{TC}}$ the cross-sectional area of the TC molecule (Eq. (2)), $P_{n}$ the probability that the structural unit at the $n$ level is stretched, $p_{\text {fail }}$ the probability distribution function of the variable, $u_{\text {fail }}\left(u_{\text {fail }}\right.$ is the displacement corresponding to the molecular chain failure), $u_{i}$ the displacement of the structural unit at the $i$ th level (correspondingly, the $(i-1)$ th level describes the structural unit that is fully uncoiled but unstretched) and $\bar{u}_{i}$ the value of $u_{i}$ that causes for the first time the complete uncoiling of the structural unit at the $i$ th level. We note that the stress generated at the $n$th level is $\sigma_{n}=\sigma_{\mathrm{TC}}$ (Eq. (2)).

The usefulness of the Annovazzi and Genna model for describing the mechanical response of SCT is now examined. The model has been implemented to evaluate the stress-strain relationship of a tendon (rat tail). ${ }^{72}$ For the purpose of this discussion, the reader is directed to Figure 4(A) for a typical (experimental) stress-strain curve of a tendon from the rat tail. A comparison of the predictions from the model with the experimental stress-strain curve of the tendon reveals that the predicted stresses corroborate the experimental result for the toe-to-heel region, the elastic deformation region and the rupture region. ${ }^{72}$ However, for the regions of yielding and plastic deformation, the stresses predicted by the model are substantially higher than those derived from experiment. This is not surprising because the model incorporates an assumption specifying that the macroscopic stress arises from the stresses generated by uniaxial extension of the ECM structural units at the respective levels. Complementing this assumption is that elastic deformation is sustained throughout the loading process until the maximum stress is reached, resulting in the rupture of the SCT. However, as we have seen in previous sections, shear sliding between the structural units in the respective levels of ECM hierarchical architecture can occur during the entire loading process. One may then argue that (i) shear sliding between the structural units in the respective levels is the main contributory factor to the macroscopic extension of the tissue during yielding and plastic deformation, and (ii) the shear sliding leads to lower macroscopic stresses (compared to the uniaxial elastic extension). Nevertheless, the mechanism by which shear sliding at the respective levels contributes to the capacity of the SCT to resist an external load acting to pull it apart remains to be resolved. Closing this gap will undoubtedly provide new insights into the interconnection of the mechanisms from molecular level upwards.

\section{Summary}

We conclude our discussion on how ECM takes up stress with a summary as follows.

(1) In general, for a SCT undergoing uniaxial extension, stress-strain relationships of ECM structural units at the respective levels of the hierarchical architecture reveal similar profiles. These profiles feature a gentle slope that reflects a slowly increasing stress with increasing strain, followed by a steeper slope that reflects rapidly increasing stress with increasing strain. Eventually, the maximum stress is reached; beyond this point, the stress decreases as the structural unit ruptures into two. 
(2) For a TC molecule, when a tensile load is applied on the molecule, initially molecular kinks are straightened. With increasing extension, this leads to bond and angular stretching of the backbone (arising from the uncoiling of the triple helix); yielding occurs in the (possibly) peptide bonds as the interstrands delaminate and finally molecular rupture occurs. The fundamental mode of deformation governing the interaction of the TC molecules addresses the nucleation of slip-pulse (intermolecular shear) and molecular stretching, leading to rupture.

(3) For a microfibril, the supertwist and interdigitation play an important role in contributing to the mechanical behavior of the collagen fibril. During normal physiological loading, interdigitation allows for microfibril sliding and this helps to regulate collagen fibril extension. The supertwist allows the microfibrils to realign themselves in the direction of the applied load acting on the collagen fibril, thus shielding the TC molecules from undue torsional stress that could disrupt the helical structure of the molecule.

(4) For collagen fibril, crosslinks and fibril lateral dimension play an important in regulating the stress-strain relationship of the fibril. Collagen fibril reinforcing the PG-rich interfibrillar matrix is regulated by different mechanisms of stress transfer during the entire loading process. These mechanisms are elastic stress transfer, intermediate modes (partial delamination, plastic PG matrix, crack initiation at the fibril end), plastic stress transfer, PG matrix rupture, collagen fibril pullout and collagen fibril rupture.
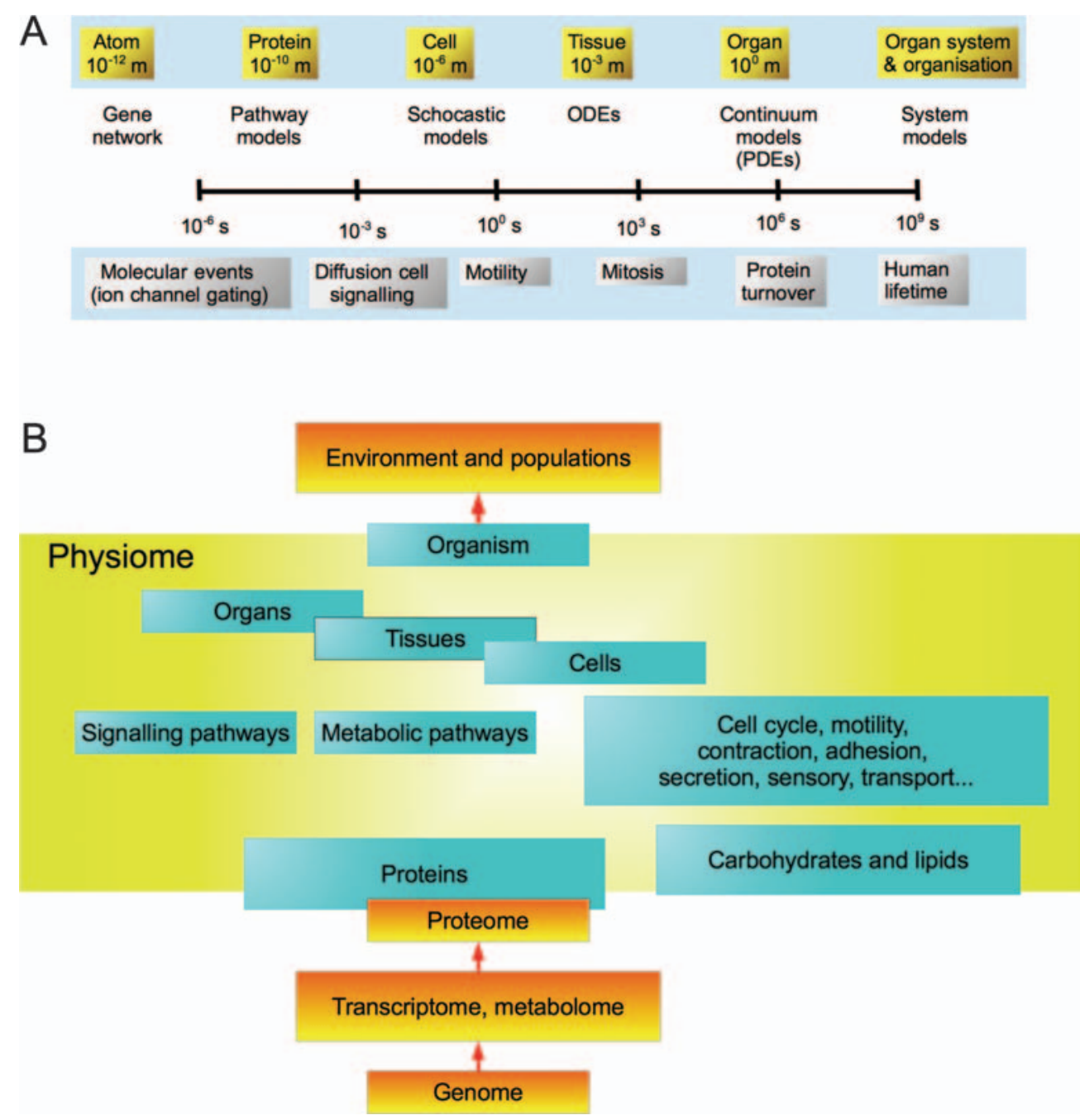

Figure 15. The Human Physiome project. (A) The scope of the Human Physiome Project as depicted by the spatial (top) and temporal (bottom) scales. The mathematical models appropriate to each spatial scale are also indicated along with the scales. (B) The Physiome in relation to the other 'omic' areas. These 'omic' areas are associated with the respective biological organization, e.g., genome is associated with the gene, transcriptome with the transcription of RNA, metabolme with metabolites and proteome with proteins. Reprinted with permission from [190], P. J. Hunter and T. K. Borg, Integration from proteins to organs: the Physiome project. Nature 4, 237 (2003). @ 2003, Nature Publishing Group. 
For both elastic and plastic stress transfer mechanisms, collagen fibrils with tapered ends lead to a more uniform distribution of axial tensile stress along the fibril than would be generated if it were uniform cylindrical. From a design perspective, taper enables the fibril to reinforce ECM more effectively that if it were uniform cylindrical because (i) a fibril with tapered ends is less likely to fracture than a uniform cylindrical fibril of the same length in a tissue subjected to the same mechanical force, (ii) the tapered fibril requires less volume of collagen than a uniform cylindrical fibril of the same length and the stress is shared more evenly along its length. However, taper provides no advantage to the fibril for reinforcing ECM when the PG-rich interfibrillar matrix eventually ruptures; tapered fibrils are more predisposed to pullout than uniform cylindrical fibrils.

(5) Collagen fibre bundles are subjected to non-uniform stresses when the SCT undergoes uniaxial extension. The mechanism of fibre-fibre sliding (and, to some extent, fibre stretching) directs the stress uptake in these bundles. Microscopic crimps are important for absorbing shock and for elastic recoil. Additionally, because collagen fibres are important for providing structural support to cells, it follows that collagen fibres play an important role in the mechanotransduction process.

(6) The mechanical properties of the ECM structural units from molecular level upwards are scale-dependent. In general, TC molecule exhibits the highest stiffness, strength and extensibility among all the known structural units. Estimates of fascicle stiffness, strength, extensibility and toughness are in good order-of-magnitude agreement with the respective mechanical properties of whole tissue. A hydrogelator model has been proposed to reconcile the scale-dependence of the mechanical properties.

(7) A framework has been proposed to facilitate the description of the mechanisms of stress uptake in ECM structural units at the respective levels of the hierarchical architecture in a consistent manner both to allow comparison of these mechanisms and make prediction concerning the interconnection of these mechanisms that can also assist in the identification of new mechanical pathways.

\section{CONCLUSION AND PROSPECTS}

There have been many studies of the structure and function of ECM of SCTs over the past several decades. These studies have covered whole tissue, collagen fibre, collagen fibril and TC molecules to address the different levels of the hierarchical architecture of ECM. This review has described an integrative approach to the subject of ECM mechanics-underlying the structure-function relationships of structural units at the respective levels of ECM-based on findings from nano to macroscopic studies. Of particular interest is the development of the ECM mechanics framework that allows for comparison of the mechanisms of reinforcement across all levels and for making prediction concerning the interconnection of these mechanisms that can also assist in the identifications of new mechanical pathways. Additionally, future study envisions a step in the direction of a more predictive application for addressing the mechanical properties of the structural units at the respective levels of the hierarchical architecture. Altogether, these developments may be further directed to tackle the major challenges in biology such as understanding the deleterious effects of tissue degeneration ${ }^{22-24}$ and injury ${ }^{22,25,26}$ on the mechanical integrity of SCTs so that effective strategies may be developed to combat the changes in ECM organization as well as to enhance the mechanical properties of SCTs via therapeutic procedures. ${ }^{25-27}$

One possible strategy for bringing us closer to this direction addresses the integration of the ECM mechanics framework into the Human Physiome project (http://physiomeproject.org/). ${ }^{190}$ The Physiome project was established with the vision to achieve a complete understanding of Human physiology. To this end, the Physiome provides a framework for an integrative approach to the understanding of the physiology of the individual based on findings derived from biochemistry, biophysics and anatomy of cells, tissues and organs by computer simulation. ${ }^{190}$ A characteristic feature of the Physiome framework is the description of mechanismsencompassing different levels of biological organization from genes, to proteins, cells, tissues, organs and finally the whole organism - over different length scales and time scales (Fig. 15(A)). ${ }^{190}$ The Physiome framework in relation to the other frameworks of biological organizations is shown in Figure 15(B). Here, the other biological organizations refer to: (i) genome (the genes encoded in DNA), (ii) transcriptome (the messenger RNA produced by gene expression), (iii) metabolme (metabolites) and (iv) proteome (proteins). Of note, population and interactions with the environment is considered to be the topmost organization. ${ }^{190}$ In accordance with the Physiome, the ECM mechanics framework may be designated within the 'tissue' category of the Physiome framework, where it will fulfill the role of providing the findings (e.g., from sequencing biomechanical data) on the key mechanical pathways that regulate the mechanical properties of ECM. The findings from ECM mechanics could be incorporated in the cells models as well as the organ models of the Physiome project. We recognise the magnitude of the challenge to bring us closer to the direction for tackling the major biological challenges in terms of the skills range and discipline range. We also recognise that we are only at the beginning of this study with a long way to go in the development of a complete set of mechanical pathways. 


\section{APPENDIX}

Table Al. Mechanical properties* of the structural unit at the respective levels of the hierarchical architecture of extracellular matrix.

\begin{tabular}{|c|c|c|c|c|}
\hline Tissue & Stiffness (MPa) & Strength (MPa) & Extensibility & Toughness (MPa) \\
\hline & \multicolumn{4}{|c|}{ Whole tissue } \\
\hline \multirow[t]{2}{*}{ Anterior cruciate ligament (Human) ${ }^{192}$} & 65.3 & 13.3 & 0.485 & 3.2 \\
\hline & 111.0 & 37.8 & 0.603 & 11.4 \\
\hline Anterior cruciate ligament (monkey) ${ }^{192}$ & 186.0 & 66.1 & 0.600 & 19.8 \\
\hline Patellar tendon (Human) ${ }^{193}$ & 305.5 & 58.3 & 0.270 & 9.9 \\
\hline Patellar tendon (Human) ${ }^{194}$ & 307.0 & 43.7 & 0.230 & 5.0 \\
\hline Patellar tendon (Human) ${ }^{195}$ & 121.0 & 47.4 & 0.170 & 4.0 \\
\hline \multirow[t]{9}{*}{ Medial collateral ligament (rabbit) ${ }^{196}$} & 700.0 & & & \\
\hline & 630.0 & 46.0 & 0.112 & 2.6 \\
\hline & 1180.0 & 84.4 & 0.108 & 4.6 \\
\hline & 740.0 & 77.7 & 0.129 & 5.0 \\
\hline & 750.0 & & & \\
\hline & 590.0 & & & \\
\hline & 950.0 & 75.8 & 0.094 & 3.6 \\
\hline & 710.0 & 78.6 & 0.133 & 5.2 \\
\hline & 520.0 & 68.9 & 0.119 & 4.1 \\
\hline \multirow[t]{2}{*}{ Patellar tendon (Human) ${ }^{197}$} & 660.0 & 64.7 & 0.140 & 4.3 \\
\hline & 504.0 & 53.6 & 0.150 & 3.7 \\
\hline \multirow[t]{2}{*}{ Achilles tendon (Human) ${ }^{198}$} & 500.0 & 50.0 & 0.050 & 1.3 \\
\hline & 1000.0 & 110.0 & 0.150 & 8.3 \\
\hline \multirow[t]{2}{*}{ Patellar tendon (rabbit) ${ }^{199}$} & 822.6 & 106.4 & 0.155 & 8.4 \\
\hline & 722.2 & 74.3 & 0.134 & 4.8 \\
\hline Patellar tendon (mouse) 200 & 462.8 & 26.1 & & \\
\hline \multirow[t]{2}{*}{ Flexor digitorum longus tendon (mouse) ${ }^{60}$} & 900.0 & 40.0 & & \\
\hline & 1500.0 & 75.0 & & \\
\hline \multirow[t]{2}{*}{ Patellar tendon (Human) 201} & 1700.0 & 51.0 & 0.061 & 1.6 \\
\hline & 2200.0 & 65.0 & 0.069 & 2.2 \\
\hline \multirow[t]{2}{*}{ Tail tendon (mouse) $)^{61,189}$} & 417.1 & 26.1 & & 3.7 \\
\hline & 691.8 & 65.9 & & 8.9 \\
\hline \multirow[t]{3}{*}{ Tail tendons (mouse) ${ }^{202}$} & 434.2 & 55.0 & 0.199 & 8.6 \\
\hline & 644.5 & 79.5 & 0.202 & 11.4 \\
\hline & \multicolumn{4}{|c|}{ Fascicle } \\
\hline Patellar tendon (rabbit) ${ }^{188}$ & 216.0 & 17.2 & 0.109 & 0.9 \\
\hline \multirow[t]{3}{*}{ Tail tendon (mouse) 203} & 250.8 & 6.4 & 0.050 & 2.6 \\
\hline & 423.5 & 26.4 & 0.111 & 2.6 \\
\hline & 530.8 & 34.8 & 0.145 & 2.6 \\
\hline Patellar tendon (rabbit) ${ }^{204}$ & 225.0 & 20.0 & 0.125 & 1.3 \\
\hline \multirow[t]{3}{*}{ Anterior cruciate ligament (swine) ${ }^{205}$} & 110.0 & 20.0 & 0.225 & 2.3 \\
\hline & 70.0 & 7.5 & 0.150 & 0.6 \\
\hline & 20.0 & 7.5 & 0.325 & 1.2 \\
\hline Posterior cruciate ligament (swine) ${ }^{205}$ & 100.0 & 20.0 & 0.325 & 3.3 \\
\hline \multirow[t]{2}{*}{ Tail tendon (mouse) ${ }^{206}$} & 202.0 & 7.0 & & \\
\hline & 467.0 & 29.0 & & \\
\hline \multirow[t]{2}{*}{ Tail tendon (mouse) ${ }^{110}$} & 200.0 & 7.5 & & \\
\hline & 450.0 & 27.5 & & \\
\hline Tail tendon (rat) ${ }^{9}$ & 571.4 & 45.0 & 0.170 & 3.8 \\
\hline \multirow[t]{3}{*}{ Tail tendon (rat) ${ }^{85}$} & & & 0.138 & \\
\hline & & & 0.190 & \\
\hline & & & 0.174 & \\
\hline \multirow[t]{3}{*}{ Tail tendon (rat) ${ }^{86}$} & & & 0.122 & \\
\hline & & & 0.141 & \\
\hline & & & 0.145 & \\
\hline Patellar tendon (rabbit) ${ }^{207}$ & 175.0 & 17.5 & 0.125 & 1.1 \\
\hline Superficial digital flexor tendon (equine) ${ }^{93}$ & 335.8 & 37.4 & 0.127 & 2.4 \\
\hline Common digital flexor tendon (equine) ${ }^{93}$ & 310.2 & 40.1 & 0.164 & 3.3 \\
\hline Anterior cruciate ligament (bovine) ${ }^{27}$ & 307.0 & & & \\
\hline
\end{tabular}


Table Al. Continued.

\begin{tabular}{|c|c|c|c|c|}
\hline Tissue & Stiffness (MPa) & Strength (MPa) & Extensibility & Toughness (MPa) \\
\hline & \multicolumn{4}{|c|}{ Collagen fibril } \\
\hline \multirow[t]{3}{*}{ Dermis (sea cucumber) ${ }^{132}$} & 300.0 & 700.0 & & \\
\hline & 400.0 & & & \\
\hline & 600.0 & & & \\
\hline \multirow[t]{3}{*}{ Achilles tendon (bovine) ${ }^{208}$} & 6000.0 & 90.0 & 0.015 & 0.7 \\
\hline & 2666.7 & 80.0 & 0.030 & 1.2 \\
\hline & 457.1 & 16.0 & 0.035 & 0.3 \\
\hline \multirow[t]{4}{*}{ Dermis (sea cucumber) ${ }^{12}$} & 666.7 & 200.0 & 0.300 & 30.0 \\
\hline & 1166.7 & 350.0 & 0.400 & 70.0 \\
\hline & 250.0 & 150.0 & 1.000 & 75.0 \\
\hline & 1200.0 & 600.0 & 0.550 & 165.0 \\
\hline Dermis (sea cucumber) ${ }^{13}$ & 470.0 & 230.0 & 0.800 & 140.0 \\
\hline MD (hydrated) ${ }^{133}$ & 1333.3 & 200.0 & 0.250 & 25.0 \\
\hline MD (Dehydrated) ${ }^{133}$ & 2000.0 & 200.0 & 0.100 & 10.0 \\
\hline \multirow[t]{4}{*}{ Achilles tendon (bovine) ${ }^{73}$} & 416.7 & 25.0 & 0.060 & 0.6 \\
\hline & 600.0 & 60.0 & 0.100 & 3.0 \\
\hline & 545.5 & 60.0 & 0.110 & 3.3 \\
\hline & \multicolumn{4}{|c|}{ Tropo-collagen (TC) molecule } \\
\hline Tail tendon (rat) ${ }^{125}$ & 9000.0 & & & \\
\hline Tail tendon (rat) ${ }^{126}$ & 5100.0 & & & \\
\hline Skin $(\text { rat })^{209}$ & 4100.0 & & & \\
\hline \multirow[t]{2}{*}{ Skin (calf) $)^{210}$} & 3000.0 & & & \\
\hline & 5100.0 & & & \\
\hline Achilles tendon (bovine) $)^{106}$ & 2666.7 & 40.0 & 0.015 & 0.3 \\
\hline Human procollagen I (fibroblast cultures) $)^{14}$ & 259.8 & 48.7 & 0.938 & 5.7 \\
\hline Type I collagen from tail tendon (rat) ${ }^{16}$ & 7086.7 & 1218.0 & 0.344 & 104.7 \\
\hline
\end{tabular}

Notes: *For an explanation of the respective mechanical properties see Section Scale-dependent mechanical properties.

Acknowledgments: Support for this work was provided by grants from the Merlion-France Programme 5.03.07 under the French Ministere des Affaires Etrangeres et Europeenes.

\section{REFERENCES}

1. A. J. Bailey, Molecular mechanisms of ageing in connective tissues. Mech. Ageing Dev. 122, 735 (2001).

2. P. P. Purslow, The structure and functional significance of variations in the connective tissue within muscle. Comp. Biochem. Physiol. Physiol. 133, 947 (2002).

3. J. A. Trotter, Structure-function considerations of muscle-tendon junctions. Comp. Biochem. Physiol. Physiol. 133, 1127 (2002).

4. D. E. Birk and R. L. Trelstad, Extracellular compartments in tendon morphogenesis: Collagen fibril, bundle, and macroaggregate formation. J. Cell Biol. 103, 231 (1986).

5. M. J. Buehler, S. Keten, and T. Ackbarow, Theoretical and computational hierarchical nanomechanics of protein materials: Deformation and fracture. Progr. Mater. Sci. 53, 1101 (2008).

6. A. Kureshi, U. Cheema, T. Alekseeva, A. Cambrey, and R. Brown, Alignment ierarchies: Engineering architecture from the nanometer to the micrometer scale. J. R. Soc. Interface 7, S707 (2010).

7. J. Diamant, A. Keller, E. Baer, M. Litt, and R. G. C. Arridge, Collagen: Ultrastructure and its relation to mechanical properties as a function of ageing. Proc. Roy. Soc. Lond. Biol. Sci. 180, 293 (1972).

8. H. R. C. Screen, D. L. Bader, D. A. Lee, and J. C. Shelton, Local strain measurement within tendon. Strain 40, 157 (2004).

9. H. R. C. Screen, D. A. Lee, D. L. Bader, and J. C. Shelton, An investigation into the effects of the hierarchical structure of tendon fascicles on micromechanical properties. Proc. IME H J. Eng. Med. 218109 (2004).

10. J. P. R. O. Orgel, J. D. San Antonio, and O. Antipova, Molecular and structural mapping of collagen fibril interactions. Connect. Tissue Res. 52, 2 (2011).

11. J. P. R. O. Orgel, O. Antipova, I. Sagi, A. Bitler, D. Qiu, R. Wang, Y. Xu, and J. D. San Antonio, Collagen fibril surface displays a constellation of sites capable of promoting fibril assembly, stability, and hemostasis. Connect. Tissue Res. 52, 18 (2011).

12. Z. L. Shen, M. R. Dodge, H. Kahn, R. Ballarini, and S. J. Eppell, Stress-strain experiments on individual collagen fibrils. Biophys. $J$. 95, 3956 (2008).

13. Z. L. Shen, M. R. Dodge, H. Kahn, R. Ballarini, and S. J. Eppell, In vitro fracture testing of submicron diameter collagen fibril specimens. Biophys. J. 99, 1986 (2010).

14. Y.-L. Sun, Z.-P. Luo, A. Fertala, and K.-N. An, Direct quantification of the flexibility of type I collagen monomer. Biochem. Biophys. Res. Comm. 295, 382 (2002).

15. Y.-L. Sun, Z.-P. Luo, A. Fertala, and K.-N. An, Stretching type II collagen with optical tweezers. J. Biomech. 37, 1665 (2004).

16. L. Bozec and M. Horton. Topography and mechanical properties of single molecules of type I collagen using atomic force microscopy. Biophys. J. 88, 4223 (2005).

17. H. R. C. Screen and S. L. Evans, Measuring strain distributions in the tendon using confocal microscopy and finite elements. Journal of Strain Analysis 44, 327 (2009).

18. G. J. Parfitt, C. Pinali, R. D. Young, A. J. Quantock, and C. Knupp, Three-dimensional reconstruction of collagen-proteoglycan interactions in the mouse corneal stroma by electron tomography. J. Struct. Biol. 170, 392 (2010).

19. M. Gasior-Glogowska, M. Komorowska, J. Hanuza, M. Ptak, and M. Kobielarza, Structural alteration of collagen 
fibres-spectroscopic and mechanical studies. Acta Bioeng. Biomech. 12, 55 (2010).

20. M. R. Doschak and R. F. Zernicke, Structure, function and adaptation of bone-tendon and bone-ligament complexes. J. Миsсuloskelet. Neuronal Interact. 5, 35 (2005).

21. A. J. Engler, S. Sen, H. L. Sweeney, and D. E. Discher, Matrix elasticity directs stem cell lineage specification. Cell 126, 677 (2006).

22. M. Pope, K. L. Goh, and M. Magnusson, Spine ergonomics. Annu. Rev. Biomed. Eng. 4, 49 (2002).

23. J. P. G. Urban and S. Roberts, Degeneration of the intervertebral disc. Arthritis Res. Ther. 5, 120 (2003).

24. H. J. Wang, A. Listrat, B. Meunier, M. Gueugneau, C. CoudyGandilhon, L. Combaret, D. Taillandier, C. Polge, D. Attaix, C. Lethias, K. Lee, K. L. Goh, and D. Bechet, Apoptosis in capillary endothelial cells in ageing skeletal muscle. Ageing Cell (2013), DOI: 10.1111/acel.12169

25. G. Fessel, J. Wernli, Y. Li, C. Gerber, and J. G. Snedeker, Exogenous collagen cross-linking recovers tendon functional integrity in an experimental model of partial tear. J. Orthop. Res. 30, 973 (2012).

26. G. Fessel, C. Gerber, and J. G. Snedeker, Potential of collagen cross-linking therapies to mediate tendon mechanical properties. J. Shoulder Elbow Surg. 21, 209 (2012).

27. K. L. Goh, S. Y. Chen, and K. Liao, A thermomechanical framework for reconciling the effects of ultraviolet radiation exposure time and wavelength on connective tissue elasticity. Biomech. Model. Mechanobiol. (2014), 10.1007/s10237-013-0551-7.

28. K. Beck and B. Brodsky, Supercoiled protein motifs: The collagen triple-helix and the alpha-helical coiled coil. J. Struc. Biol. 122, 17 (1998).

29. K. E. Kadler, C. Baldock, J. Bella, and R. P. Boot-Handford, Collagens at a glance. J. Cell Sci. 120, 1955 (2007).

30. S. Ozbek, P. G. Balasubramanian, R. Chiquet-Ehrismann, R. P. Tucker, and J. C. Adams, The evolution of extracellular matrix. Mol. Biol. Cell 21, 4300 (2010).

31. D. E. Birk, E. I. Zycband, D. A. Winkelmann, and R. L. Trelstad, Collagen fibrillogenesis in situ: Fibril segments are intermediates in matrix assembly. Proc. Natl. Acad. Sci. USA 86, 4549 (1989).

32. K. E. Kadler, D. F. Holmes, J. A. Trotter, and J. A. Chapman, Collagen fibril formation. Biochem. J. 316, 1 (1996).

33. K. E. Kadler, A. Hill, and E. G. Canty-Laird, Collagen fibrillogenesis: Fibronectin, integrins, and minor collagens as organizers and nucleators. Curr. Opin. Cell Biol. 20, 495 (2008).

34. G. Zhang, B. B. Young, Y. Ezura, M. Favata, and L. J. Soslowsky, S. Chakravarti, D. E. Birk, Development of tendon structure and function: Regulation of collagen fibrillogenesis. J. Musculoskel. Neuronal Interact. 5, 5 (2005).

35. J. B. Dacks, A. A. Peden, and M. C. Field, Evolution of specificity in the eukaryotic endomembrane system. Int. J. Biochem. Cell Biol. 41, 330 (2009).

36. E. G. Canty, Y. Lu, R. S. Meadows, M. K. Shaw, D. F. Holmes, and K. E. Kadler, Coalignment of plasma membrane channels and protrusions (fibripositors) specifies the parallelism of tendon. J. Cell Biol. 165, 553 (2004).

37. E. G. Canty and K. E. Kadler, Procollagen trafficking, processing and fibrillogenesis. Journal of Cell Science 118, 1341 (2005).

38. D. J. Hulmes, Building Collagen molecules, fibrils, and suprafibrillar structures. J. Struct. Biol. 137, 2 (2002).

39. G. N. Ramachandran and G. Sasisekharan, Structure of collagen. Nature 176, 593 (1955).

40. Z. Kapacee, S. H. Richardson SH, Y. Lu, T. Starborg, D. F. Holmes, K. Baar, and K. E. Kadler, Tension is required for fibripositor formation. Matrix Biol. 27, 371 (2008).

41. D. F. Holmes, J. A. Chapman, D. J. Prockop, and K. E. Kadler, Growing tips of Type I collagen fibrils formed in vitro are nearparaboloidal in shape, implying a reciprocal relationship between accretion and diameter. Proc. Natl. Acad. Sci. USA 89, 9855 (1992).
42. D. F. Holmes, H. K. Graham, and K. E. Kadler, Collagen fibrils forming in developing tendon show an early and abrupt limitation in diameter at the growing tips. J. Mol. Biol. 283, 1049 (1998).

43. D. F. Holmes, A. Tait, N. W. Hodson, M. J. Sherratt, and K. E. Kadler, Growth of collagen fibril seeds form embryonic tendon: Fractured fibril ends nucleate new tip growth. J. Mol. Biol. 399, 9 (2010).

44. J. A. Trotter, K. E. Kadler, and D. F. Holmes, Echinoderm collagen fibrils grow by surface-nucleation-and-propagation from both centers and ends. J. Mol. Biol. 300, 531 (2000).

45. S. G. M. Uzel and M. J. Buehler, Molecular structure, mechanical behavior and failure mechanism of the C-terminal cross-link domain in type I collagen. J. Mech. Behav. Biomed. Mater. 4, 153 (2011).

46. H. K. Graham, D. F. Holmes, R. B. Watson, and K. E. Kadler, Identification of collagen fibril fusion during vertebrate tendon morphogenesis. The process relies on unipolar fibrils and is regulated by collagen-proteoglycan interaction. J. Mol. Biol. 295, 891 (2000).

47. T. Starborg, Y. Lu, A. Huffman, D. F. Holmes, and K. E. Kadler, Electron microscope 3D reconstruction of branched collagen fibrils in vivo. Scand. J. Med. Sci. Sports 19, 547 (2009).

48. K. G. Danielson, H. Baribault, D. F. Holmes, H. Graham, K. E. Kadler, and R. V. Iozzo, Targeted disruption of decorin leads to abnormal collagen fibril morphology and skin fragility. J. Cell Biol. 136, 729 (1997)

49. D. R. Keene, J. D. San Antonio, R. Mayne, D. J. McQuillan, G. Sarris, S. A. Santoro, and R. V. Iozzo, Decorin binds near the C terminus of Type I collagen. J. Biol. Chem. 275, 21801 (2000).

50. P. N. Lewis, C. Pinali, R. D. Young, K. M. Meek, A. J. Quantock, and C. Knupp, Structural interactions between collagen and proteoglycans are elucidated by three-dimensional electron tomography of bovine cornea. Structure 18, 239 (2010).

51. J. E. Scott, Elasticity in extracellular matrix 'shape modules' of tendon, cartilage, etc. A sliding proteoglycan-filament model. J. Physiol. 553, 35 (2003).

52. J. P. R. O. Orgel, A. Eid, O. Antipova, J. Bella, and J. E. Scott, Decorin core protein (Decoron) shape complements collagen fibril surface structure and mediates its binding. PLOS ONE 4, e7028 (2009).

53. S. Perumal, O. Antipova, and J. Orgel, Collagen fibril architecture, domain organization, and triple-helical conformation govern its proteolysis. Proc. Natl. Acad. Sci. USA 105, 2824 (2008).

54. A. J. Quantock and R. D. Young, Development of the corneal stroma, and the collagen-proteoglycan associations that help define its structure and function. Dev. Dynam. 237, 2607 (2008).

55. K. M. Meek and C. Boote, The use of X-ray scattering techniques to quantify the orientation and distribution of collagen in the corneal stroma. Progr. Retin. Eye Res. 28, 369 (2009).

56. A. H. Plaas, L. A. West, E. J. Thonar, Z. A. Karcioglu, C. J. Smith, G. K. Klintworth, and V. C. Hascall, Altered fine structures of corneal and skeletal keratan sulfate and chondroitin/dermatan sulfate in macular corneal dystrophy. J. Biol. Chem. 276, 39788 (2001).

57. J. E. Scott, Structure and function in extracellular matrices depend on interactions between anionic glycosaminoglycans. Pathol. Biol. 49, 284 (2001).

58. A. Asanbaeya, J. Tam, B. L. Schumacher, S. M. Klisch, K. Masuda, R. L. Sah, Articular cartilage tensile integrity: Modulation by matrix depletion is maturation-dependent. Arch. Biochem. Biophys. 474, 175 (2008).

59. J.-W. Handgraaf and F. Zerbetto, Molecular dynamics study of onset of water gelation around the collagen triple helix. Protein Struct. Funct. Genet. 64, 711 (2006).

60. G. Zhang, Y. Ezura, I Chervoneva, P. S. Robinson, D. P. Beason, E. T. Carine, L. J. Soslowsky, R. V. Iozzo, and D. E. Birk, Decorin 
regulates assembly of collagen fibrils and acquisition of biomechanical properties during tendon development. J. Cell. Biochem. 98, 1436 (2006).

61. K. L. Goh, D. F. Holmes, Y. Lin, P. P. Purslow, K. E. Kadler, D. Bechet, and T. J. Wess, Bimodal collagen fibril diameter distributions direct age-related variations in tendon resilience and resistance to rupture. J. Appl. Physiol. 113, 1 (2012).

62. A. Redaelli, S. Vesentini, M. Soncini, P. Vena, S. Mantero, and F. M. Montevecchi, Possible role of decorin glycosaminoglycans in fibril to fibril force transfer in relative mature tendons-A computational study from molecular to microstructural level. J. Biomech. 36,1555 (2003).

63. P. G. Scott, P. A. McEwan, C. M. Dodd, E. M. Bergmann, P. N. Bishop, and J. Bella, Crystal structure of the dimeric protein core of decorin, the archetypal small leucine-rich repeat proteoglycan. Proc. Natl. Acad. Sci. USA 101, 15633 (2004).

64. P. G. Scott, J. G. Grossmann, C. M. Dodd, J. K. Sheehan, and P. N. Bishop, Light and X-ray scattering show decorin to be a dimer in solution. J. Biol. Chem. 278, 18353 (2003).

65. S. Goldoni, R. T. Owens, D. J. McQuillan, Z. Shriver, R. Sasisekharan, D. E. Birk, S. Campbell, and R. V. Iozzo, Biologically active decorin is a monomer in solution. J. Biol. Chem. 279, 6606 (2004).

66. P. G. Scott, C. M. Dodd, E. M. Bergmann, J. K. Sheehan, and P. N. Bishop, Crystal structure of the biglycan dimer and evidence that dimerization is essential for folding and stability of class I small leucine-rich repeat proteoglycans. J. Biol. Chem. 281, 13324 (2006).

67. M. Islam, J. Gor, S. J. Perkins, Y. Ishikawa, H. P. Bächinger, and E. Hohenester, The concave face of decorin mediates reversible dimerization and collagen binding. J. Biol. Chem. 288, 35526 (2013).

68. J. Rainey and M. Goh, A statistically derived parameterization for the collagen triple-helix. Protein Sci. 11, 2748 (2002).

69. S. Iwasaki, Y. Hosaka, T. Iwasaki, K. Yamamoto, A. Nagayasu, H. Ueda, Y. Kokai, and K. Takehana, The modulation of collagen fibril assembly and its structure by decorin: An electron microscopic study. Arch. Histol. Cytol. 71, 37 (2008).

70. V. Ottani, D. Martini, M. Franchi, A. Ruggeri, and M. Raspanti, Hierarchical structures in fibrillar collagens. Micron 33, 587 (2002).

71. J. P. R. O. Orgel, T. C. Irving, A. Miller, and T. J. Wess, Microfibrillar structure of type I collagen in situ. Proc. Natl. Acad. Sci. USA 103, 9001 (2006).

72. L. Annovazzi and F. Genna, An engineering, multiscale constitutive model for fibre-forming collagen in tension. J. Biomed. Mater. Res. 92A, 254 (2010).

73. L. Yang, K. O. van der Werf, P. J. Dijkstra, J. Feijen, and M. L. Bennink, Micromechanical analysis of native and cross-linked collagen type I fibrils supports the existence of microfibrils. J. Mech. Behav. Biomed. Mater. 6, 148 (2012).

74. D. J. S. Hulmes, A. Miller, D. A. D. Parry, K. A. Piez, and J. Woodhead-Galloway, Analysis of the primary structure of collagen for the origins of molecular packing. J. Mol. Biol. 79, 137 (1973).

75. D. J. S. Hulmes, T. J. Wess, D. J. Prockop, and P. Fratzl, Radial packing, order, and disorder in collagen fibrils. Biophys. J. 68, 1661 (1995).

76. D. F. Holmes and K. E. Kadler, The $10+4$ microfibril structure of thin cartilage fibrils. Proc. Natl. Acad. Sci. USA 103, 17249 (2006).

77. D. F. Holmes, C. J. Gilpin, C. Baldock, U. Ziese, A. J. Koster, and K. E. Kadler, Corneal collagen fibril structure in three dimensions: Structural insights into fibril assembly, mechanical properties, and tissue organization. Proc. Natl. Acad. Sci. USA 98, 7307 (2001).

78. D. E. Birk, R. A. Hahn, C. Y. Linsenmayer, and E. I. Zycband, Characterization of collagen fibril segments from chicken embryo cornea, dermis and tendon. Matrix Biol. 15, 111 (1996).
79. D. E. Birk, E. I. Zycband, S. Woodruff, D. A. Winkelmann, and R. L. Trelstad, Collagen fibrillogenesis in situ: Fibril segments become long fibrils as the developing tendon matures. Dev. Dynam. 208, 291 (1997).

80. J. A. Trotter and T. Koob, Collagen and proteoglycan in a sea urchin ligament with mutable mechanical properties. Cell Tissue Res. 258, 527 (1989).

81. J. E. deVente, G. E. Lester, J. A. Trotter, and L. E. Dahners, Isolation of intact collagen fibrils from healing ligament. J. Electron Microsc. 46, 353 (1997).

82. J. A. Trotter, J. A. Chapman, K. E. Kadler, and D. F. Holmes, Growth of sea cucumber collagen fibrils occurs at the tips and centers in a coordinated manner. J. Mol. Biol. 284, 1417 (1998).

83. D. Silver, J. Miller, R. Harrison, D. J. Prockop, Helical model of nucleation and propagation to account for the growth of type I collagen fibrils from symmetrical pointed tips: A special example of self-assembly of rod-like monomers. Proc. Natl. Acad. Sci. USA 89, 9860 (1992).

84. D. H. Elliott, Structure and function of mammalian tendon. Biol. Rev. 40, 392 (1965).

85. H. R. C. Screen, J. C. Shelton, V. H. Chhaya, M. V. Kayser, D. L. Bader, and D. A. Lee, The influence of noncollagenous matrix components on the micromechanical environment of tendon fascicles. Ann. Biomed. Eng. 33, 1090 (2005).

86. H. R. C. Screen, V. H. Chhaya, S. E. Greenwald, D. L. Bader, D. A. Lee, and J. C. Shelton, The influence of swelling and matrix degradation on the microstructural integrity of tendon. Acta Biomaterialia 2, 505 (2006).

87. J. R. Ralphs, Cell biology of tendons. Eur. Cells Mater. 4, 39 (2002).

88. S. S. Chi, J. B. Rattner, P. Sciore, R. Boorman, and I. K. Y. Lo, Gap junctions of the medial collateral ligament: Structure, distribution, associations and function. J. Anat. 207, 145 (2005).

89. J. M. Mellado, J. Calmet, M. Olona, J. Gine, and A. Sauri, Magnetic resonance imaging of anterior cruciate ligament tears: Reevaluation of quantitative parameters and imaging findings including a simplified method for measuring the anterior cruciate ligament angle. Knee Surg. Sports Traumatol. Arthrosc. 12, 217 (2004).

90. H. Steckel, G. Vadala, D. Davis, and F. H. Fu, 2D and 3D 3-tesla magnetic resonance imaging of the double bundle structure in anterior cruciate ligament anatomy. Knee Surg. Sports Traumatol. Arthrosc. 14, 1151 (2006).

91. V. S. Cheong, C. L. Poh, K. S. A. Yew, D. T. T. Lie, K. Sheah, and K. L. Goh, Magnetic resonance imaging of the human anterior cruciate ligament: Three-dimensional computer reconstruction and structural analysis. J. Med. Imaging Health Inf. 2, 378 (2012).

92. O. Basso, D. P. Johnson, and A. A. Amis, The anatomy of the patellar tendon. Knee Surg. Sports Traumatol. Arthrosc. 9, 2 (2001).

93. C. T. Thorpe, C. P. Udeze, H. L. Birch, P. D. Clegg, and H. R. C. Screen, Specialization of tendon mechanical properties results from interfascicular differences. J. Roy. Soc. Interface 9, 3108 (2012).

94. M. L. Wood, G. E. Lester, and L. E. Dahners, Collagen fibre sliding during ligament growth and contracture. J. Orthop. Res. 16, 430 (1998).

95. S. Hirokawa and R. Tsuruno, Three-dimensional deformation and stress distribution in an analytical/computational model of the anterior cruciate ligament. J. Biomech. 33, 1069 (2000).

96. M. Benjamin, H. Toumi, J. R. Ralphs, G. Bydder, T. M. Best, and S. Milz, Where tendons and ligaments meet bone: Attachment sites ('entheses') in relation to exercise and/or mechanical load. J. Anat. 208, 471 (2006).

97. R. Newsham-West, H. Nicholson, M. Walton, and P. Milburn, Long-term morphology of a healing bone-tendon interface: A histological observation in the sheep model. J. Anat. 210, 318 (2007).

98. T. Finni, Structural and functional features of human muscletendon unit. Scand. J. Med. Sci. Sports 16, 147 (2006). 
99. B. Charvet, F. Ruggiero, and D. Le Guellec, The development of the myotendinous junction. A review. Muscles Ligaments Tendons J. 2, 53 (2012).

100. C. Zhu, G. Bao, and N. Wang, Cell mechanics: Mechanical response, cell adhesion, and molecular deformation. Аnпи. Rev. Biomed. Eng. 2, 189 (2000).

101. M. J. Sharratt, Tissue elasticity and the ageing elastic fibre. Age 31, 305 (2009)

102. C. M. Kielty, M. J. Sherratt, and C. A. Shuttleworth, Elastic fibres. J. Cell Sci. 115, 2817 (2002).

103. J. Gosline, M. Lillie, E. Carrington, P. Guerette P, C. Ortlepp, and K. Savage, Elastic proteins: Biological roles and mechanical properties. Phil. Trans. Roy. Soc. Lond. B 357, 121 (2002).

104. T. M. Ritty, K. Ditsios, and B. C. Starcher, Distribution of the elastic fiber and associated proteins in flexor tendon reflects function. Anat. Rec. 268, 430 (2002).

105. K. D. Smith, A. Vaughan-Thomas, D. G. Spiller, J. F. Innes, P. D. Clegg, and E. J. Comerford, The organization of elastin and fibrillins 1 and 2 in the cruciate ligament complex. J. Anat. 218, 600 (2011).

106. N. Sasaki and S. Odajima, Stress-strain curve and Young's modulus of a collagen molecule as determined by the X-ray diffraction technique. J. Biomech. 29, 655 (1996).

107. N. Sasaki and S. Odajima, Elongation mechanism of collagen fibrils and force-strain relationship of tendon at each level of structural hierarchy. J. Biomech. 29, 1131 (1996).

108. G. Azangwe, K. J. Mathias, and D. Marshall, Macro and microscopic examination of the ruptured surfaces of anterior cruciate ligaments of rabbits. J. Bone. Joint Surg. 82-B, 450 (2000).

109. G. Azangwe, K. Fraser, K. J. Mathias, and A.M. Siddiqui, In vitro monitoring of rabbit anterior cruciate ligament damage by acoustic emission. Med. Eng. Phys. 22, 279 (2000).

110. P. S. Robinson, T. W. Lin, P. R. Reynolds, K. A. Derwin, R. V. Iozzo, and L. J. Soslowsky, Strain-rate sensitive mechanical properties of tendon fascicles from mice with genetically engineered alterations in collagen and decorin. J. Biomech. Eng. 126, 252 (2004).

111. H. R. C. Screen, J. Seto, S. Krauss, P. Boeseckec, and H. S. Gupta, Extrafibrillar diffusion and intrafibrillar swelling at the nanoscale are associated with stress relaxation in the soft collagenous matrix tissue of tendons. Soft Matter. 7, 11243 (2011).

112. R. Puxkandl, I Zizak, O. Paris, J. Keckes, W. Tesch, S. Bernstorf, P. Purslow, and P. Fratzl, Viscoelastic properties of collagen: Synchrotron radiation investigations and structural model. Phil. Trans. Roy. Soc. Lond. B 357, 191 (2002).

113. D. M. Elliott, P. S. Robinson, J. A. Gimbel, J. J. Sarver, J. A. Abboud, R. V. Iozzo, and L. J. Soslowsky, Effect of altered matrix proteins on quasilinear viscoelastic properties in transgenic mouse tail tendons. Ann. Biomed. Eng. 31, 599 (2003).

114. A. Gautieri, S. Vesentini, A. Redaelli, and M. J. Buehler, Viscoelastic properties of model segments of collagen molecules. Matrix Biol. 31, 141 (2012).

115. A. Minajeva, M. Kulke, J. M. Fernandez, and W. A. Linke, Unfolding of titin domains explains the viscoelastic behavior of skeletal myofibrils. Biophys. J. 80, 1442 (2001).

116. S. Hayward and R. A. Lee, Improvements in the analysis of domain motions in proteins from conformational change: DynDom version 1.50. J. Mol. Graph. Model. 21, 181 (2002).

117. G. P. Poornam, A. Matsumoto, H. Ishida, and S. Hayward, A method for the analysis of domain movements in large biomolecular complexes. Proteins 76, 201 (2009).

118. S. Zhuang, Q. Peng, Y. Cao, and H. Li, Modulating the mechanical stability of extracellular matrix protein tenascin-C in a controlled and reversible fashion. J. Mol. Biol. 390, 820 (2009).

119. P. J. in't Veld and M. J. Stevens, Simulation of the mechanical strength of a single collagen molecule. Biophys. J. 95, 33 (2008).
120. P. Fratzl, K. Misof, I. Zizak, G. Rapp, H. Amenitsch, and S. Bernstorff, Fibrillar structure and mechanical properties of collagen. J. Struct. Biol. 122, 119 (1998).

121. Y.-N. Wang, C. Galiotis, and D. L. Bader, Determination of molecular changes in soft tissues under strain using laser Raman microscopy. J. Biomech. 33, 483 (2000).

122. T. Gutsmann, G. E. Fantner, J. H. Kindt, M. Venturoni, S. Danielsen, and P. K. Hansma, Force spectroscopy of collagen fibers to investigate their mechanical properties and structural organization. Biophys. J. 86, 3186 (2004).

123. M. J. Buehler, Nature designs tough collagen: Explaining the nanostructure of collagen fibrils. Proc. Natl. Acad. Sci. USA 103, 12285 (2006).

124. M. J. Buehler, Molecular architecture of collagen fibrils: A critical length scale for tough fibrils. Curr. Appl. Phys. 8, 440 (2008).

125. R. Harley, D. James, A. Miller, and J. W. White, Phonons and the elastic moduli of collagen and muscle. Nature 267, 285 (1977).

126. S. Cusack and A. Miller, Determination of the elastic constants of collagen by Brillouin tight scattering. J. Mol. Biol. 135, 39 (1979).

127. E. Makareeva, W. A. Cabral, J. C. Marini, and S. Leikin, Molecular mechanism of $\alpha 1$ (I)-osteogenesis imperfecta/Ehlers-Danlos syndrome. J. Biol. Chem. 281, 6463 (2006).

128. I. T. S. Li and G. C. Walker, Interfacial free energy governs single polystyrene hain collapse in water and aqueous solutions. $J$. Am. Chem. Soc. 132, 6530 (2010).

129. I. T. S. Li and G. C. Walker, Signature of hydrophobic hydration in a single polymer. Proc. Natl. Acad. Sci. USA 108, 16527 (2011).

130. C. Bustamante, S. B. Smith, J. Liphardt, and D. Smith, Singlemolecule studies of DNA mechanics. Curr. Opin. Struct. Biol. 10, 279 (2000).

131. M. J. Buehler and S. Y. Wong, Entropic elasticity controls nanomechanics of single tropocollagen molecules. Biophys. J. 93, 37 (2007).

132. S. J. Eppell, B. N. Smith, H. Kahn, and R. Ballarini, Nano measurements with micro-devices: Mechanical properties of hydrated collagen fibrils. J. R. Soc. Interface 3, 117 (2006).

133. A. Gautieri, S. Vesentini, A. Redaelli, and M. J. Buehler, Hierarchical structure and nanomechanics of collagen microfibrils from the atomistic scale up. Nano Lett. 11, 757 (2011).

134. H. Tang, M. J. Buehler, and B. Moran, A constitutive model of soft tissue: From nanoscale collagen to tissue continuum. Ann. Biomed. Eng. 37, 1117 (2009).

135. Y. Tang, R. Ballarini, M. J. Buehler, and S. J. Eppell, Deformation micromechanisms of collagen fibrils under uniaxial tension. J. Roy. Soc. Interface 7, 839 (2010).

136. M. J. Buehler, Nanomechanics of collagen fibrils under varying cross-link densities: Atomistic and continuum studies. J. Mech. Behav. Biomed. Mater. 1, 59 (2008).

137. K. L. Goh, R. M. Aspden, K. J. Mathias, and D. W. L. Hukins, Effect of fibre shape on the stresses within fibres in fibre-reinforced composite materials. Proc. Roy. Soc. Lond. Math. Phys. Sci. 455, 3351 (1999).

138. K. L. Goh, R. M. Aspden, K. J. Mathias, and D. W. L. Hukins, Finite-element analysis of the effect of material properties and fibre shape on stresses in an elastic fibre embedded in an elastic matrix in a fibre-composite material. Proc. Roy. Soc. Lond. Math. Phys. Sci. 460, 2339 (2004).

139. K. L. Goh, R. M. Aspden, and D. W. L. Hukins, Review: Finite element analysis of stress transfer in short-fibre composite materials. Compos. Sci. Technol. 64, 1091 (2004).

140. K. L. Goh, J. R. Meakin, R. M. Aspden, and D. W. L. Hukins, Influence of fibril taper on the function of collagen to reinforce extracellular matrix. Proc. Roy. Soc. Lond. Biol. Sci. 272, 1979 (2005). 
141. K. L. Goh, J. R. Meakin, R. M. Aspden, and D. W. L Hukins, Stress transfer in collagen fibrils reinforcing connective tissues: Effects of collagen fibril slenderness and relative stiffness. J. Theor. Biol. 245, 305 (2007).

142. H. L. Cox, The elasticity and strength of paper and other fibrous materials. Bri. J. Appl. Phys. 3, 72 (1952).

143. A. Kelly and M. H. Macmillan, Strong Solids, 3rd edn., Clarendon Press, Oxford (1986)

144. A. S. Carrara and F. J. McGarry, Matrix and interface stresses in a discontinuous fibre composite model. J. Compos. Mater. 2, 222 (1968).

145. R. M. Aspden, Fibre reinforcing by collagen in cartilage and soft connective tissues. Proc. Roy. Soc. Lond. B 258, 195 (1994).

146. K. L. Goh, J. R. Meakin, and D. W. L. Hukins, Influence of fibre taper on the interfacial shear stress in fibre-reinforced composite materials during elastic stress transfer. Compos. Interfac. 17, 75 (2010).

147. J. A. Trotter, F. A. Thurmond, and T. Koob, Molecular structur and functional morphology of echinoderm collagen fibrils. Cell Tissue Res. 275, 451 (1994)

148. D. Tripathi, F. P. Chen, and F. R. Jones, The effect of matrix plasticity on the stress fields in a single filament composite and the value of interfacial shear strength obtained from the fragmentation test. Proc. Roy. Soc. Lond. Math. Phys. Sci. 452, 621 (1996).

149. D. M. Schuster and E. Scala, The mechanical interaction of sapphire whiskers with a birefringent matrix. TMS-AIME 230, 1635 (1964).

150. R. B. Nath, D. N. Fenner, and C. Galiotis, The progressional approach to interfacial failure in carbon reinforced composites: Elasto-plastic finite element modeling of interface cracks. Compos. Appl. Sci. Manuf. 31, 929 (2000).

151. S. A. Hayes, R. Lane, and F. R. Jones, Fibre/matrix stress transfer through a discrete interphase, Part 1: Single-fibre model composites. Compos. Appl. Sci. Manuf. 32, 379 (2001).

152. D. R. J. Owen and L. F. Lyness. Investigation of bond failure in fibre-reinforced materials by the finite element method. Fibre Sci. Technol. 5, 129 (1972).

153. S. Sirivedin, D. N. Fenner, R. B. Nath, and C. Galiotis, Matrix crack propagation criteria for model short-carbon fibre/epoxy composites. Compos. Sci. Technol. 60, 2835 (2000).

154. S. E. Szczesny and D. M. Elliott, Interfibrillar shear stress is the loading mechanism of collagen fibrils in tendon. Acta Biomaterialia (2014), http://dx.doi.org/10.1016/j.actbio.2014.01.032.

155. G. Azangwe, K. J. Mathias, and D. Marshall, The effect of flexion angle on the macro and microscopic appearance of the rupture surface of the ACL of rabbits. Knee 8, 29 (2001).

156. G. Azangwe, K. J. Mathias, and D. Marshall, Preliminary comparison of the rupture of human and rabbit anterior cruciate ligaments. Clin. Biomech. 16, 913 (2001).

157. G. Azangwe, K. J. Mathias, and D. Marshall, The effect of torsion on the appearance of the rupture surface of the ACL of rabbits. Knee 9, 31 (2002).

158. X. W. Ng, D. W. L. Hukins, and K. L. Goh, Influence of fibre taper on the work of fibre pull-out in short fibre composite fracture. J. Mater. Sci. 45, 1086 (2010).

159. J. A. Bennett and R. J. Young, The effect of fibre-matrix adhesion upon crack bridging in fibre reinforced composites. Compos. Appl. Sci. Manuf. 29, 1071 (1998).

160. X. Zhang, H. Y. Liu, Y. W. Mai, and X. X. Diao, On steady-state fibre pull-out I-The stress field. Compos. Sci. Technol. 59, 2179 (1999).

161. D. J. Bannister, M. C. Andrews, A. J. Cervenka, and R. J. Young, Analysis of the single-fibre pull-out test by means of Raman spectroscopy: Part II. Micromechanics of deformation for an aramid/epoxy system. Compos. Sci. Technol. 53, 411 (1995).
162. G. Rauchs, M. Preuss, and P. J. Withers, Micromechanical analysis of internal stress development during single-fibre fragmentation testing of Ti/SiCf. Acta Mater. 50, 2477 (2002).

163. D. Tripathi and F. R. Jones, Measurement of the load-bearing capability of the fibre/matrix interface by single-fibre fragmentation. Compos. Sci. Technol. 57, 925 (1997).

164. D. Agarwal and L. J. Broutman, Analysis and Performance of Fibre Composites, 2nd edn., Wiley, New York (1990).

165. K. L. Goh, R. M. Aspden, and D. W. L. Hukins, Critical length of collagen fibrils in extracellular matrix. J. Theor. Biol. 223, 259 (2003).

166. D. E. Birk, J. F. Southern, E. I. Zycband, J. T. Fallon, and R. L. Trelstad, Collagen fibril bundles: A branching assembly unit in tendon morphogenesis. Development 107, 437 (1989).

167. C. Boote, S. Dennis, R. H. Newton, H. Puri, and K. M. Meek, Collagen fibrils appear more closely packed in the prepupillary cornea: Optical and biomechanical implications. Investig. Ophthalmol. Vis. Sci. 44, 2941 (2003).

168. K. L. Goh, A. M. A. Huq, R. M. Aspden, and D. W. L. Hukins, Nano-fibre critical length depends on shape. Adv. Compos. Lett. 17, 131 (2008).

169. A. A. Dunkman, M. R. Buckley, M. J. Mienaltowski, S. M. Adams, S. J. Thomas, L. Satchell, A. Kumar, L. Pathmanathan, D. P. Beason, R. V. Iozzo, D. E. Birk, and L. J. Soslowsky, Decorin expression is important for age-related changes in tendon structure and mechanical properties. Matrix Biol. 32, 3 (2013).

170. X. Liu, M.-L. Yeh, J. L. Lewis, and Z.-P. Luo, Direct measurement of the rupture force of single pair of decorin interactions. Biochem. Biophys. Res. Comm. 338, 1342 (2005).

171. P. Ciarletta and M. B. Amar, A finite dissipative theory of temporary interfibrillar bridges in the extracellular matrix of ligaments and tendons. J. R. Soc. Interface 6, 909 (2009).

172. T. J. Lujan, C. J. Underwood, H. B. Henninger, B. M. Thompson, and J. A. Weiss. Effect of dermatan sulfate glycosaminoglycans on the quasi-static material properties of the human medial collateral ligament. J. Orthop. Res. 25, 894 (2007).

173. T. J. Lujan, C. J. Underwood, N. T. Jacobs, and J. A. Weiss. Contribution of glycosaminoglycans to viscoelastic tensile behavior of human ligament. J. Appl. Physiol. 106, 423 (2009).

174. G. Fessel and J. G. Snedeker, Evidence against proteoglycan mediated collagen fibril load transmission and dynamic viscoelasticity in tendon. Matrix Biol. 28, 503 (2009).

175. G. Fessel and J. G. Snedeker, Equivalent stiffness after glycosaminoglycan depletion in tendon-An ultra-structural finite element model and corresponding experiments. J. Theor. Biol. 268, 77 (2011).

176. A. Edwards, A. M. J. Bull, and A. A. Amis, The attachments of the anteromedial and posterolateral fibre bundles of the anterior cruciate ligament. Knee Surg. Sports Traumatol. Arthrosc. 16, 29 (2008).

177. T. Zantop, W. Petersen, J. K. Sekiya, V. Musahl, and F. H. Fu, Anterior cruciate ligament anatomy and function relating to anatomical reconstruction. Knee Surg. Sports Traumatol. Arthrosc. 14, 982 (2006).

178. C. Sommer, N. Friederich, and W. Muller, Improperly placed anterior cruciate ligament grafts: Correlation between radiological parameters and clinical results. Knee Surg. Sports Traumatol. Arthrosc. 8, 207 (2000).

179. R. de Wreede and J. R. Ralphs, Deposition of collagenous matrices by tendon fibroblasts in vitro: A comparison of fibroblast behavior in pellet cultures and a novel three-dimensional long-term scaffoldless culture system. Tissue Eng. 15, 2707 (2009).

180. K. A. Hansen, J. A. Weiss, and J. K. Barton, Recruitment of tendon crimp with applied tensile strain. J. Biomech. Eng. 124, 72 (2002).

181. R. F. Ker, The implications of the adaptable fatigue quality of tendons for their construction, repair and function. Comp. Biochem. Physiol. Physiol. 133, 987 (2002). 
182. M. Franchi, M. Fini, M. Quaranta, V. De Pasquale, M. Raspanti, G. Giavaresi, V. Ottani, and A. Ruggeri, Crimp morphology in relaxed and stretched rat Achilles tendon. J. Anat. 210, 1 (2007).

183. M. Benjamin, E. Kaiser, and S. Milz, Structure-function relationships in tendons: A review. J. Anat. 212, 211 (2008).

184. T. M. Hammoudi and J. S. Temenoff, Biomaterials for Tissue Engineering Applications, edited by J. A. Burdick and R. L. Mauck, Springer-Verlag, Wien (2011), pp. 307-341.

185. A. Herchenhan, N. S. Kalson, D. F. Holmes, P. Hill, K. E. Kadler, and L. Margetts, Tenocyte contraction induces crimp formation in tendon-like tissue. Biomech. Model. Mechanobiol. 11, 49 (2012).

186. T. A. Jarvinen, T. L. Jarvinen, P. Kannus, L. Jozsa, and M. Jarvinen, Collagen fibres of the spontaneously ruptured human tendons display decreased thickness and crimp angle. J. Orthop. Res. 22, 1303 (2004).

187. F. J. Alenghat and D. E. Ingber, Mechanotransduction: All signals point to cytoskeleton, matrix, and integrins. Science STKE 119, 1 (2002).

188. E. Yamamoto, K. Hayashi, and N. Yamamoto, Mechanical properties of collagen fascicles from the rabbit patellar tendon. J. Biomech. Eng. 121, 124 (1999).

189. K. L. Goh, D. F. Holmes, H.-Y. Lin, S. Richardson, K. E. Kadler, P. P. Purslow, and T. J. Wess, Ageing changes in the tensile properties of tendons: Influence of collagen fibril volume fraction. J. Biomech. Eng. 130, 021011 (2008).

190. P. J. Hunter and T. K. Borg, Integration from proteins to organs: The Physiome project. Nature 4, 237 (2003).

191. J. Kastelic, A. Galeski, and E. Baer, The multicomposite structure of tendon. Connect. Tissue Res. 6, 11 (1978).

192. F. R. Noyes and E. S. Grood, The strength of the anterior cruciate ligament in humans and rhesus monkeys. J. Bone Joint. Surg. 58, 1074 (1976)

193. D. L. Butler, E. S. Grood, and F. R. Noyes, Effects of structure and strain measurement technique on the material properties of young human tendons and fascia. J. Biomech. 17, 579 (1984).

194. R. C. Haut, A. C. Powlison, O. W. Rutherford, and J. R. Kateley, Some effects of donor age and sex on the mechanical properties of patellar tendon graft tissues. ASME Adv. Bioeng. 8, 75 (1988).

195. E. P. France, L. E. Paulos, I. D. Rosenberg, and C. D. Harner, Prosthetic Ligament Reconstruction of the Knee, edited by M. J. Friedman and R. D. Ferkel, W. B. Saunders, Philadelphia (1988), pp. $180-185$.

196. S. L.-Y. Woo, K. J. Ohlan, and J. A. Weiss, Aging and sex-related changes in the biomechanical properties of the rabbit medial collateral ligament. Mech. Ageing Dev. 56, 129 (1990).
197. G. A. Johnson, D. M. Tramaglini, R. E. Levine, K. Ohno, N.-Y. Choi, and S. L.-Y. Woo, Tensile and viscoelastic properties of human patellar tendon. J. Orthop. Res. 12, 796 (1994).

198. T. A. L. Wren, S. A. Yerby, G. S. Beaupre, and D. R. Carter, Mechanical properties of the human achilles tendon. Clin. Biomech. 16, 245 (2001)

199. M. R. Dressler, D. L. Butler, R. Wenstrup, H. A. Awad, F. Smith, and G. P. Boivin, A potential mechanism for age-related declines in patellar tendon biomechanics. J. Orthop. Res. 20, 1315 (2002).

200. T. W. Lin, L. Cardenas, and L. J. Soslowsky, Tendon properties in interleukin-4 and interleukin-6 knockout mice. J. Biomech. 38, 99 (2005).

201. C. Couppe, P. Hansen, M. Kongsgaard, V. Kovanen, C. Suetta, P. Aagaard, M. Kjær, and S. P. Magnusson, Mechanical properties and collagen cross-linking of the patellar tendon in old and young men. J. Appl. Physiol. 107, 880 (2009).

202. K. L. Goh, Y. Chen, S. M. Chou, A. Listrat, D. Bechet, and T. J. Wess, Effects of frozen storage temperature on the elasticity of tendons from a small murine model. Animal 4, 1613 (2010).

203. K. A. Derwin and L. J. Soslowsky, A quantitative investigation of the structure-function relationship in a tendon fascicle model. J. Biomech. Eng. 121, 598 (1999).

204. E. Yamamoto, S. Tokura, N. Yamamoto, and K. Hayashi, Mechanical properties of collagen fascicles from in situ frozen and stressshielded rabbit patellar tendons. Clin. Biomech. 15, 284 (2000).

205. S. Hirokawa and T. Sakoshita, An experimental study of the microstructures and mechanical properties of swine cruciate ligaments. JSME International Journal Series C 46, 1417 (2003).

206. P. S. Robinson, T. W. Lin, A. F. Jawad, R. V. Iozzo, and L. J. Soslowsky, Investigating tendon fascicle structure-function relationships in a transgenic-age mouse model using multiple regression models. Ann. Biomed. Eng. 32, 924 (2004).

207. E. Yamamoto, D. Kogawa, S. Tokura, and K. Hayashi, Biomechanical response of collagen fascicles to restressing after stress deprivation during culture. J. Biomech. 40, 2063 (2007).

208. J. A. J. van der Rijt, K. O. van der Werf, M. L. Bennink, P. J. Dijkstra, and J. Feijen, Micromechanical testing of individual collagen fibrils. Macromol. Biosci. 6, 697 (2006).

209. F. H. M. Nestler, S. Hvidt, and J. D. Ferry, Flexibility of collagen determined from dilute solution viscoelastic measurements. Biopolymers 22, 1747 (1983).

210. H. Hofman, T. Voss, K. Kuhn, and J. Engel, Localization of flexible sites in thread-like molecules from electron micrographs. Comparison of interstitial, basement membrane and intima collagens. J. Mol. Biol. 25, 325 (1984). 